\title{
AB INITIO CALCULATIONS OF THE REACTION MECHANISMS FOR METAL NITRIDE DEPOSITION FROM ORGANO-METALLIC PRECURSORS ONTO FUNCTIONALIZED SELF-ASSEMBLED MONOLAYERS
}

\author{
Mohit Haran, James R. Engstrom and Paulette Clancy* \\ School of Chemical and Biomolecular Engineering \\ Cornell University \\ Ithaca, NY 14853 \\ USA \\ email: pqc1@cornell.edu
}

Supporting Information (Pages66)

*Author to whom correspondence should be addressed 
Gaussian 03, Revision C.02, Frisch, M. J. et. al., Gaussian, Inc., Wallingford CT, 2004.

\section{Complete Ref. 38, “More Frisch et al.”}

38. Gaussian 03, Revision C.02, Frisch, M. J.; Trucks, G. W.; Schlegel, H. B.; Scuseria, G. E.; Robb, M. A.; Cheeseman, J. R.; Montgomery, Jr., J. A.; Vreven, T.; Kudin, K. N.; Burant, J. C.; Millam, J. M.; Iyengar, S. S.; Tomasi, J.; Barone, V.; Mennucci, B.; Cossi, M.; Scalmani, G.; Rega, N.; Petersson, G. A.; Nakatsuji, H.; Hada, M.; Ehara, M.; Toyota, K.; Fukuda, R.; Hasegawa, J.; Ishida, M.; Nakajima, T.; Honda, Y.; Kitao, O.; Nakai, H.; Klene, M.; Li, X.; Knox, J. E.; Hratchian, H. P.; Cross, J. B.; Bakken, V.; Adamo, C.; Jaramillo, J.; Gomperts, R.; Stratmann, R. E.; Yazyev, O.; Austin, A. J.; Cammi, R.; Pomelli, C.; Ochterski, J. W.; Ayala, P. Y.; Morokuma, K.; Voth, G. A.; Salvador, P.; Dannenberg, J. J.; Zakrzewski, V. G.; Dapprich, S.; Daniels, A. D.; Strain, M. C.; Farkas, O.; Malick, D. K.; Rabuck, A. D.; Raghavachari, K.; Foresman, J. B.; Ortiz, J. V.; Cui, Q.; Baboul, A. G.; Clifford, S.; Cioslowski, J.; Stefanov, B. B.; Liu, G.; Liashenko, A.; Piskorz, P.; Komaromi, I.; Martin, R. L.; Fox, D. J.; Keith, T.; Al-Laham, M. A.; Peng, C. Y.; Nanayakkara, A.; Challacombe, M.; Gill, P. M. W.; Johnson, B.; Chen, W.; Wong, M. W.; Gonzalez, C.; and Pople, J. A.; Gaussian, Inc., Wallingford CT, 2004. 


$$
\begin{gathered}
k=\tau\left(\frac{k_{n} T Q_{t}^{\neq}(T)}{h Q_{r}(T)}\right) \exp \left(-\frac{E_{a}}{R T}\right) \\
\tau=1+\frac{1}{24}\left(\frac{h v}{k_{b} T}\right)^{2}
\end{gathered}
$$

In precursor mediated deposition, the adduct has two translation degree of freedom in the plane of surface and one rotational degree of freedom along axes normal to surface. The activated structure has only vibrational degree of freedom. Here surface partition function is assumed 1

$$
\begin{gathered}
Q_{t}^{\#}=Q_{\text {transition }}^{\#}=Q_{v}^{\#}(T) \\
Q_{r}=Q_{\text {adduct }}=Q_{\text {tran }}^{2 / 3}(T) Q_{\text {rot }}\left(Q_{\text {TDMAT-surface }}\right)_{\text {vib }} \\
k_{r}=\tau\left(\frac{k_{n} T\left(Q_{t}^{\neq}(T)\right)_{v}}{h\left(Q_{\text {TDMAT-trans }}(T)\right)^{0.66} * Q_{\text {rot }} *\left(Q_{T D M A T \text {-surface }}(T)\right)_{v}}\right) \exp \left(-\frac{E_{a}}{R T}\right)
\end{gathered}
$$

Reference:

www.catalysis.nl/kica/files/02\%20Reaction\%20Rate\%20Theory\%20of\%20Surface\%20Reactions. pdf 
Table S1: Comparison of results for various model chemistries for increasingly bulky precursors (TiA, TDMAT and TDEAT). (Energies given in $\mathrm{kcal} / \mathrm{mol}$ )

\begin{tabular}{|c|c|c|c|c|c|}
\hline & \multicolumn{3}{|c|}{ B3LYP } & \multicolumn{2}{|c|}{ MP2 } \\
\hline respect to adduct) & $\begin{array}{c}\text { 6- } \\
311 G(D)\end{array}$ & $6-311+G(D)$ & $6-311++G(D, P)$ & $6-311 G(D)$ & $6-311+G(D)$ \\
\hline $\begin{array}{c}\text { TiA } \\
\text { TDMAT } \\
\text { TDEAT }\end{array}$ & $\begin{array}{l}21.77 \\
23.13 \\
21.23 \\
\end{array}$ & $\begin{array}{l}24.49 \\
23.40\end{array}$ & $\begin{array}{l}23.95 \\
21.77\end{array}$ & $\begin{array}{l}23.95 \\
23.95 \\
\\
23.95 \\
\end{array}$ & $\begin{array}{l}24.22 \\
25.03\end{array}$ \\
\hline Adduct/Complex & & & & & \\
\hline $\begin{array}{c}\text { TiA } \\
\text { TDMAT } \\
\text { TEMAT }\end{array}$ & $\begin{array}{r}-11.16 \\
-1.90 \\
4.90\end{array}$ & $\begin{array}{l}-7.35 \\
1.90\end{array}$ & $\begin{array}{l}-5.99 \\
3.27\end{array}$ & $\begin{array}{l}-18.23 \\
-11.70 \\
-4.08 \\
\end{array}$ & $\begin{array}{c}-16.60 \\
-12.25 \\
0.00\end{array}$ \\
\hline
\end{tabular}




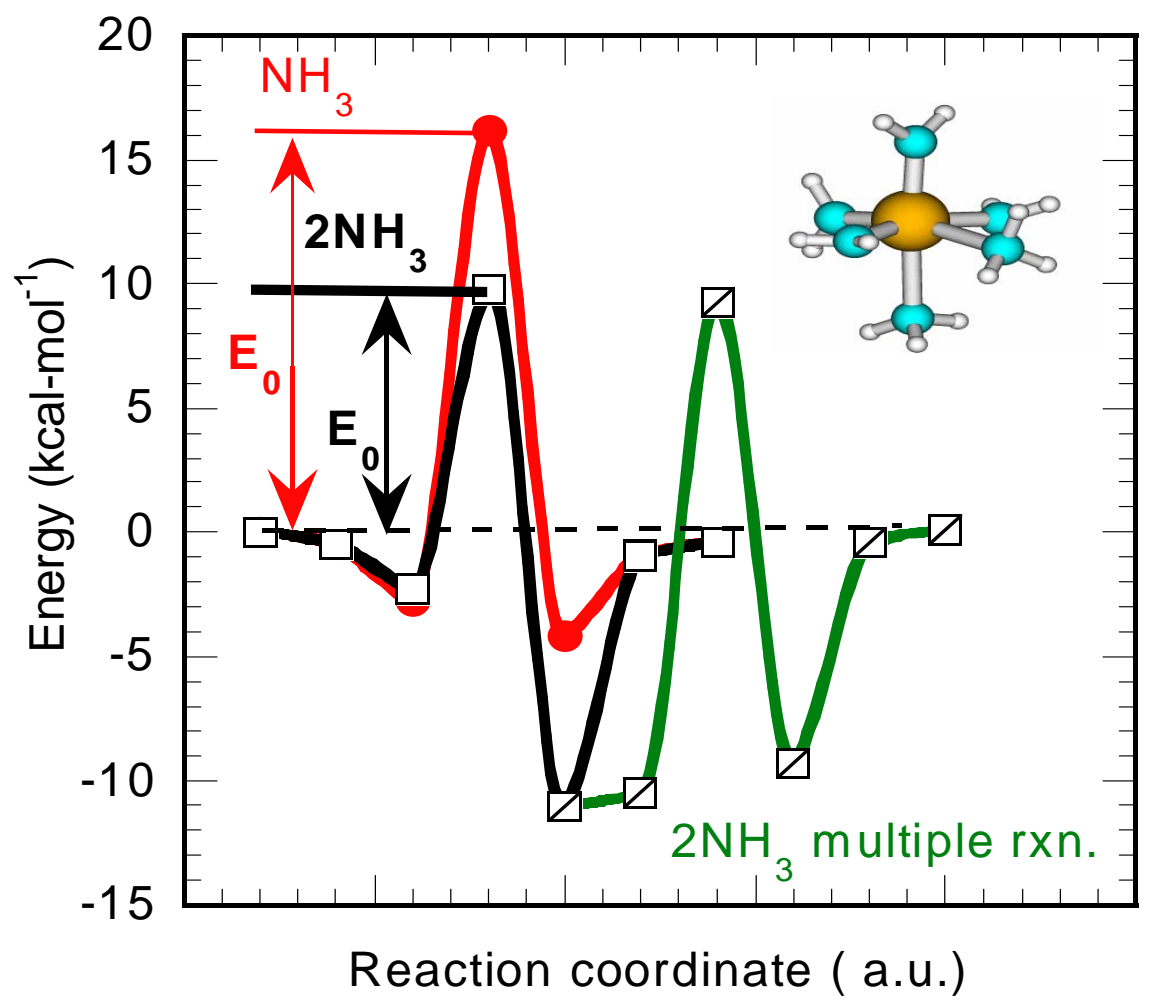

Figure S1 PED for the deposition of TDMAT with a single ammonia molecule (uncatalyzed, shown in red) and two ammonia molecules (catalyzed, shown in black and green). Insert represents the physisorbed complex with two ammonia molecules. 
Data Points for All The figures

Figure 2:

\begin{tabular}{|c|cc|cc|cc|}
\hline \multirow{2}{*}{$\mathrm{RXH}($ Terminal Group) } & \multicolumn{2}{|c|}{$\mathrm{NH}_{2}$} & \multicolumn{2}{c|}{$\mathrm{OH}$} & \multicolumn{2}{c|}{$\mathrm{SH}$} \\
\hline $\mathrm{R}=\mathrm{CH}_{3} \mathrm{CH}_{2}$ & $\mathrm{~B} 3 \mathrm{LYP}$ & $\mathrm{MP} 2$ & $\mathrm{~B} 3 \mathrm{LYP}$ & $\mathrm{MP} 2$ & B3LYP & $\mathrm{MP} 2$ \\
\cline { 2 - 7 } $\mathrm{Ti}\left(\mathrm{NH}_{2}\right)_{4}+\mathrm{RXH}$ (Vacuum) & 0.00 & 0.00 & 0.00 & 0.00 & 0.00 & 0.00 \\
$\mathrm{Ti}\left(\mathrm{NH}_{2}\right)_{4}---\mathrm{HXR}$ (Adduct) & -4.79 & -14.96 & -5.11 & -13.30 & -1.80 & -7.67 \\
$\mathrm{Ti}\left(\mathrm{NH}_{2}\right)_{4}---\mathrm{H}--\mathrm{XR}$ (Transition) & 15.82 & 5.66 & 2.68 & -6.87 & 10.21 & 0.33 \\
$\mathrm{Ti}(\mathrm{NH} 2)_{3}-\mathrm{RX}--\mathrm{H}--\mathrm{NH}_{2}$ & -5.51 & -16.81 & -21.88 & -35.33 & -12.86 & -26.98 \\
$\mathrm{Ti}\left(\mathrm{NH}_{2}\right)_{3} \mathrm{RX}+\mathrm{NH}_{3}$ (Product) & -0.24 & -2.91 & -17.63 & -23.37 & -7.96 & -14.03 \\
\hline
\end{tabular}

Figure 3:

\begin{tabular}{|c|cc|cc|cc|}
\hline \multirow{2}{*}{ XH(Terminal Group) } & \multicolumn{2}{|c|}{$\mathrm{NH} 2$} & \multicolumn{2}{c|}{$\mathrm{OH}$} & \multicolumn{2}{c|}{$\mathrm{SH}$} \\
\cline { 2 - 7 } & B3LYP & MP2 & B3LYP & MP2 & B3LYP & MP2 \\
\hline TDMAT+RXH(Vacuum) & 0.00 & 0.00 & 0.00 & 0.00 & 0.00 & 0.00 \\
TDMAT---HXR (Adduct) & 6.19 & -7.94 & -0.64 & -8.61 & 1.09 & -4.57 \\
TDMAT---H--XR(Transition) & 21.79 & 9.38 & 7.49 & -4.41 & 10.94 & 0.90 \\
Ti(N($\left.\left(\mathrm{CH}_{3}\right)_{2}\right)_{3}-\mathrm{RX}--\mathrm{H}--\mathrm{N}\left(\mathrm{CH}_{3}\right)_{2}$ & 3.87 & -7.88 & -18.77 & -24.49 & -4.98 & -21.05 \\
$\mathrm{Ti}\left(\mathrm{N}\left(\mathrm{CH}_{3}\right)_{2}\right)_{3} \mathrm{RX}+\mathrm{HN}\left(\mathrm{CH}_{3}\right)_{2}$ & -0.39 & 0.00 & -17.44 & -17.39 & -10.57 & -7.14 \\
\hline
\end{tabular}

Figure 4:

\begin{tabular}{|c|c|c|c|c|c|}
\hline & \multicolumn{2}{|c|}{ Amine } & \multicolumn{2}{c|}{ Hydroxyl } & Thiol \\
\hline $\mathrm{MR}_{1}$ & ZrA & TDMAZ & ZrA & TDMAZ & ZrA \\
$\mathrm{MR}_{1}+\mathrm{R}_{2} \mathrm{XH}$ & 0.00 & 0.00 & 0.00 & 0.00 & 0.00 \\
$\mathrm{MR}_{1}--\mathrm{R}_{2} \mathrm{XH}$ & -11.71 & -1.96 & -10.14 & -2.31 & -2.62 \\
$\mathrm{MR}_{1}--\mathrm{H}--$ & & & & & \\
$\mathrm{R}_{2} \mathrm{XH}$ & 8.31 & 14.21 & -6.90 & -0.18 & 4.81 \\
$\mathrm{MR}_{2} \mathrm{NH}-\mathrm{H}--$ & & -5.66 & -32.11 & -24.34 & -22.85 \\
$\mathrm{XR}$ & -11.23 & -5.18 & -20.82 & -19.74 & -7.90 \\
$\mathrm{MR}_{2} \mathrm{NH}+\mathrm{R}_{1} \mathrm{H}$ & -0.16 & -0.69 & -20.82 \\
\hline
\end{tabular}


Figure 5:

\begin{tabular}{|c|c|c|c|c|c|}
\hline SAM & $\mathrm{C}_{2} \mathrm{~F}_{5} \mathrm{NH}_{2}$ & $\mathrm{CF}_{2} \mathrm{CFNH}_{2}$ & $\mathrm{CH}_{3} \mathrm{CF}_{2} \mathrm{NH}_{2}$ & $\mathrm{CF}_{3} \mathrm{NH}_{2}$ & $\mathrm{CH}_{3} \mathrm{CH}_{2} \mathrm{NH}_{2}$ \\
\hline Reactant & 0 & 0 & 0 & 0 & 0 \\
Adduct & -6.00 & -5.11 & -8.31 & -5.59 & -4.79 \\
Transition & 9.51 & 12.10 & 9.05 & 8.55 & 15.82 \\
Adduct & -14.56 & -11.90 & -15.37 & -14.44 & -5.51 \\
Product & -4.30 & -4.01 & -4.42 & -4.77 & -0.24 \\
\hline
\end{tabular}

Figure 6:

\begin{tabular}{|c|c|c|c|c|c|c|}
\hline \multirow{2}{*}{$\begin{array}{c}\text { Terminal group } \\
\text { No. of SAM molecules }\end{array}$} & \multicolumn{2}{|c|}{$\mathrm{NH}_{2}$} & \multicolumn{2}{|c|}{$\mathrm{OH}$} & \multicolumn{2}{|c|}{$\mathrm{SH}$} \\
\hline & One & Two & One & Two & One & Two \\
\hline Reactant & 0.00 & 0.00 & 0.00 & 0.00 & 0.00 & 0.00 \\
\hline Adduct & -9.32 & -12.17 & -11.80 & -20.28 & -5.69 & -9.37 \\
\hline Transition & 8.14 & 8.31 & -5.05 & -9.31 & 3.00 & -3.47 \\
\hline Adduct & -11.78 & -13.98 & -31.77 & -38.07 & -19.36 & -21.16 \\
\hline Product & -2.57 & -4.99 & -22.31 & -29.94 & -9.98 & -12.06 \\
\hline
\end{tabular}


Figure 8

\begin{tabular}{|c|c|c|c|c|c|c|c|c|}
\hline & \multicolumn{2}{|c|}{ Electronic energy } & \multicolumn{6}{|c|}{ Free energy } \\
\hline & \multirow[b]{2}{*}{ B3LYP } & \multirow[b]{2}{*}{ MP2 } & \multirow[b]{2}{*}{198} & \multirow[b]{2}{*}{298} & \multirow[b]{2}{*}{398} & \multicolumn{3}{|c|}{ Shifted } \\
\hline & & & & & & 198 & 298 & 398 \\
\hline $\mathrm{Ti}\left(\mathrm{N}\left(\mathrm{CH}_{3}\right)_{2}\right)_{4}+2 \mathrm{RNH}_{2}{ }^{*}$ & 0 & 0 & 0 & 0 & 0 & 50 & 50 & 50 \\
\hline $\mathrm{Ti}\left(\mathrm{N}\left(\mathrm{CH}_{3}\right)_{2}\right)_{4}---2 \mathrm{RNH}_{2}{ }^{*}$ & -2.66 & -11.63 & -0.5 & 4.9 & 10.2 & 49.5 & 54.9 & 60.21 \\
\hline $\mathrm{Ti}\left(\mathrm{N}\left(\mathrm{CH}_{3}\right)_{2}\right)_{4}---\mathrm{H}---\mathrm{NHRRNH}_{2}{ }^{*}$ & 18.36 & 6.12 & 15.7 & 21.6 & 27.4 & 65.75 & 71.61 & 77.39 \\
\hline 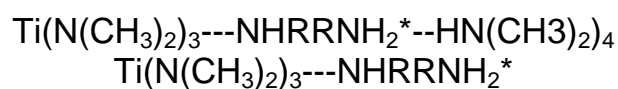 & 4.96 & -15.27 & 0.5 & 7.9 & 15.2 & 50.55 & 57.87 & 65.18 \\
\hline$\left.+\mathrm{HN}(\mathrm{CH} 3)_{2}\right)_{4}$ & -1.05 & -5.51 & -4.1 & -4 & -3.9 & 45.92 & 45.99 & 46.06 \\
\hline $\mathrm{RNH}---\mathrm{H}---\mathrm{Ti}\left(\mathrm{N}\left(\mathrm{CH}_{3}\right)_{2}\right)_{3} \mathrm{NHR}$ & 23.73 & 15.41 & 17.4 & 19.8 & 22.3 & 67.38 & 69.76 & 72.33 \\
\hline$\left.\left(\mathrm{CH}_{3}\right)_{2}\right) \mathrm{NH}---\mathrm{RNH}---\mathrm{Ti}\left(\mathrm{N}\left(\mathrm{CH}_{3}\right)_{2}\right)_{2} \mathrm{NHR}$ & 10.87 & -29.35 & 8.9 & 10 & 11.2 & 58.9 & 59.96 & 61.17 \\
\hline$\left.\left(\mathrm{CH}_{3}\right)_{2}\right) \mathrm{NH}+\mathrm{RNHTi}\left(\mathrm{N}\left(\mathrm{CH}_{3}\right)_{2}\right)_{2} \mathrm{NHR}$ & 6.78 & -0.59 & 0.26 & -3.47 & -6.99 & 50.26 & 46.53 & 43.01 \\
\hline $\mathrm{RNHTi}\left(\mathrm{N}\left(\mathrm{CH}_{3}\right)_{2}\right)-\mathrm{N}\left(\mathrm{CH}_{3}\right)_{2}--\mathrm{H}-\mathrm{NR}$ & 36.24 & 36.4 & 26.8 & 22.73 & 18.88 & 76.8 & 72.73 & 68.88 \\
\hline $\mathrm{RNHTi}\left(\mathrm{N}\left(\mathrm{CH}_{3}\right)_{2}\right)=\mathrm{NR}--\mathrm{HN}\left(\mathrm{CH}_{3}\right)_{2}$ & 12.72 & -7.24 & -6.42 & -10.81 & -15.02 & 43.58 & 39.19 & 34.98 \\
\hline $\mathrm{RNHTi}\left(\mathrm{N}\left(\mathrm{CH}_{3}\right)_{2}\right)=\mathrm{NR}+\mathrm{HN}\left(\mathrm{CH}_{3}\right)_{2}$ & 33.74 & 22.46 & 13.81 & 4.6 & -4.28 & 63.81 & 54.6 & 45.72 \\
\hline
\end{tabular}


Figure 9:

\begin{tabular}{|c|c|c|c|c|c|c|c|c|c|c|}
\hline & \multicolumn{3}{|c|}{ Electronic energy } & \multicolumn{6}{|c|}{ Free Energy } & \\
\hline & \multirow[b]{2}{*}{ Favored } & & & \multicolumn{3}{|c|}{198} & \multicolumn{3}{|c|}{398} & \\
\hline & & \multicolumn{2}{|c|}{ Side } & Favored & sic & & favored & sid & & \\
\hline 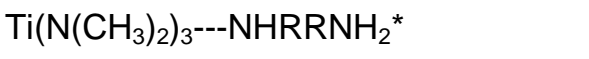 & 0.00 & 0.00 & & 80.00 & 80.00 & & 80.00 & 80.00 & & 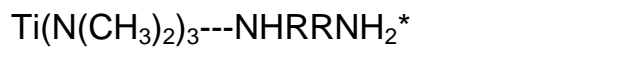 \\
\hline RNH---H---Ti( $\left(\mathrm{N}\left(\mathrm{CH}_{3}\right)_{2}\right)_{3} \mathrm{NHR}$ & 24.78 & 34.10 & & 101.46 & 115.52 & & 106.27 & 117.31 & & $\left.\mathrm{Ti}\left(\mathrm{N}\left(\mathrm{CH}_{3}\right)_{2}\right) 2--\mathrm{N}\left(\mathrm{CH}_{3}\right)_{2}\right)---\mathrm{H}--\mathrm{NRRNH}_{2}{ }^{*}$ \\
\hline$\left.\left(\mathrm{CH}_{3}\right)_{2}\right) \mathrm{NH}---\mathrm{RNH}---\mathrm{Ti}\left(\mathrm{N}\left(\mathrm{CH}_{3}\right)_{2}\right)_{2} \mathrm{NHR}$ & 11.92 & 11.92 & & 92.98 & 84.98 & & 95.11 & 87.11 & & $\mathrm{Ti}\left(\mathrm{N}\left(\mathrm{CH}_{3}\right)_{2}\right) 2=\mathrm{NRRNH}_{2}{ }^{*}---\mathrm{HN}\left(\mathrm{CH}_{3}\right)_{2}$ \\
\hline$\left.\left(\mathrm{CH}_{3}\right)_{2}\right) \mathrm{NH}+\mathrm{RNHTi}\left(\mathrm{N}\left(\mathrm{CH}_{3}\right)_{2}\right)_{2} \mathrm{NHR}$ & 7.82 & 47.76 & 7.82 & 77.14 & 117.19 & 77.14 & 69.74 & 107.24 & 69.74 & $\begin{array}{l}\mathrm{Ti}\left(\mathrm{N}\left(\mathrm{CH}_{3}\right)_{2}\right) 2=\mathrm{NRRNH}_{2}{ }^{*}+\mathrm{HN}\left(\mathrm{CH}_{3}\right)_{2} \\
\mathrm{RNHTi}\left(\mathrm{N}\left(\mathrm{CH}_{3}\right)_{2}\right)-\mathrm{CH}_{2}---\mathrm{H}--\end{array}$ \\
\hline $\mathrm{RNHTi}\left(\mathrm{N}\left(\mathrm{CH}_{3}\right)_{2}\right)--\mathrm{N}\left(\mathrm{CH}_{3}\right)_{2}--\mathrm{H}--\mathrm{NR}$ & 37.28 & & 48.17 & 110.88 & & 114.54 & 102.81 & & 106.23 & $\mathrm{~N}\left(\mathrm{CH}_{3}\right)_{2} \mathrm{RNH}$ \\
\hline $\mathrm{RNHTi}\left(\mathrm{N}\left(\mathrm{CH}_{3}\right)_{2}\right)=\mathrm{NR}-\mathrm{HN}\left(\mathrm{CH}_{3}\right)_{2}$ & 13.77 & & 33.31 & 70.46 & & 97.85 & 61.72 & & 89.19 & $\mathrm{RNHTi}\left(\mathrm{N}\left(\mathrm{CH}_{3}\right)_{2}\right)-\mathrm{CH}_{2}-\mathrm{NHR}---\mathrm{HN}\left(\mathrm{CH}_{3}\right)_{2}$ \\
\hline $\mathrm{RNHTi}\left(\mathrm{N}\left(\mathrm{CH}_{3}\right)_{2}\right)=\mathrm{NR}+\mathrm{HN}\left(\mathrm{CH}_{3}\right)_{2}$ & 34.78 & & 53.22 & 90.69 & & & 72.46 & & & $\mathrm{RNHTi}\left(\mathrm{N}\left(\mathrm{CH}_{3}\right)_{2}\right)-\mathrm{CH}_{2}-\mathrm{NHR}+\mathrm{HN}\left(\mathrm{CH}_{3}\right)_{2}$ \\
\hline $\mathrm{RN}-\mathrm{H}-\mathrm{-}\left(\mathrm{N}\left(\mathrm{CH}_{3}\right)_{2}\right) \mathrm{Ti}=\mathrm{NR}$ & 77.25 & & & 129.39 & & & 111.38 & & & \\
\hline $\left.\mathrm{RN}=\mathrm{Ti}=\mathrm{NR}+\mathrm{HN}\left(\mathrm{CH}_{3}\right)_{2}\right)$ & 44.76 & & & 99.23 & & & 78.72 & & & \\
\hline
\end{tabular}


Figure 11:

\begin{tabular}{|c|c|c|c|c|c|}
\hline & \multicolumn{2}{|c|}{ Electronic energy } & \multicolumn{3}{|c|}{ Free energy } \\
\hline & B3LYP & MP2 & 198 & 298 & 398 \\
\hline $\mathrm{Ti}\left(\mathrm{N}\left(\mathrm{CH}_{3}\right)_{2}\right)_{4}+2 \mathrm{ROH}^{*}$ & 0 & 0 & 0 & 0 & 0 \\
\hline $\mathrm{Ti}\left(\mathrm{N}\left(\mathrm{CH}_{3}\right)_{2}\right)_{4}---2 \mathrm{ROH}^{*}$ & -10.16 & -23.24 & -8.32 & -2.1 & 4.02 \\
\hline $\mathrm{Ti}\left(\mathrm{N}\left(\mathrm{CH}_{3}\right)_{2}\right)_{4}{ }^{---} \mathrm{H}---\mathrm{ORROH}^{*}$ & 0.74 & -14.54 & 0.26 & 7.74 & 15.18 \\
\hline 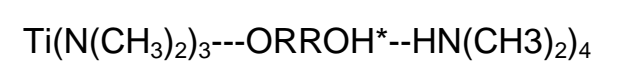 & -27.08 & -44.07 & 27.76 & 21.07 & -14.46 \\
\hline $\left.\mathrm{Ti}\left(\mathrm{N}\left(\mathrm{CH}_{3}\right)_{2}\right)_{3}---\mathrm{OHRROH}{ }^{*}+\mathrm{HN}(\mathrm{CH} 3)_{2}\right)_{4}$ & -25.56 & -30.75 & 25.04 & -23.8 & -22.52 \\
\hline RO---H---Ti( $\left(\mathrm{N}\left(\mathrm{CH}_{3}\right)_{2}\right)_{3} \mathrm{OR}$ & -18.52 & -24.25 & 20.16 & 18.24 & -16.22 \\
\hline$\left.\left(\mathrm{CH}_{3}\right)_{2}\right) \mathrm{NH}---\mathrm{RO}---\mathrm{Ti}\left(\mathrm{N}\left(\mathrm{CH}_{3}\right)_{2}\right)_{2} \mathrm{OR}$ & -38.49 & -47.74 & -40.5 & $\begin{array}{c}- \\
38.53\end{array}$ & -36.42 \\
\hline$\left.\left(\mathrm{CH}_{3}\right)_{2}\right) \mathrm{NH}+\mathrm{ROTi}\left(\mathrm{N}\left(\mathrm{CH}_{3}\right)_{2}\right)_{2} \mathrm{OR}$ & -43.32 & -41.47 & $\begin{array}{c}- \\
45.87\end{array}$ & $\begin{array}{c}- \\
49.92\end{array}$ & -53.77 \\
\hline $\mathrm{ROTi}\left(\mathrm{N}\left(\mathrm{CH}_{3}\right)_{2}\right)-\mathrm{CH}_{2}---\mathrm{H}-\mathrm{N}\left(\mathrm{CH}_{3}\right)_{2} \mathrm{RO}$ & -3.18 & 0.12 & $\begin{array}{c}-7.2 \\
-\end{array}$ & 11.07 & -14.7 \\
\hline $\mathrm{ROTi}\left(\mathrm{N}\left(\mathrm{CH}_{3}\right)_{2}\right)-\mathrm{CH}_{2}-\mathrm{OR}---\mathrm{HN}\left(\mathrm{CH}_{3}\right)_{2}$ & -21.36 & -20.19 & 23.71 & 27.54 & -31.16 \\
\hline $\mathrm{ROTi}\left(\mathrm{N}\left(\mathrm{CH}_{3}\right)_{2}\right)-\mathrm{CH}_{2}-\mathrm{OR}+\mathrm{HN}\left(\mathrm{CH}_{3}\right)_{2}$ & -0.77 & 14.16 & -0.01 & -9.04 & -17.74 \\
\hline
\end{tabular}


Figure 12

\begin{tabular}{|c|c|c|c|c|c|c|c|c|}
\hline & \multicolumn{2}{|c|}{$\begin{array}{l}\text { Electronic } \\
\text { energy }\end{array}$} & \multicolumn{6}{|c|}{ Free energy } \\
\hline & B3LYP & MP2 & 198 & 298 & 398 & 198 & 298 & 398 \\
\hline $\mathrm{Ti}\left(\mathrm{N}\left(\mathrm{CH}_{3}\right)_{2}\right) 4+2 \mathrm{RSH}^{*}$ & 0.00 & 0.00 & 0.00 & 0.00 & 0.00 & 40.00 & 40.00 & 40.00 \\
\hline $\mathrm{Ti}\left(\mathrm{N}\left(\mathrm{CH}_{3}\right)_{2}\right)_{4^{---}} \mathrm{RSH}^{*}$ & 1.68 & -9.51 & 4.35 & 10.84 & 17.30 & 44.35 & 50.84 & 57.30 \\
\hline $\mathrm{Ti}\left(\mathrm{N}\left(\mathrm{CH}_{3}\right)_{2}\right)_{4}{ }^{---} \mathrm{H}---\mathrm{SRRSH}^{*}$ & 5.68 & -3.95 & 8.77 & 15.44 & 22.13 & 48.77 & 55.44 & 62.13 \\
\hline $\left.\mathrm{Ti}\left(\mathrm{N}\left(\mathrm{CH}_{3}\right)_{2}\right)_{3}{ }^{---} \mathrm{SRRSH}^{*}-\mathrm{HN}\left(\mathrm{CH}_{3}\right)_{2}\right)_{4}$ & -7.92 & -26.98 & 5.84 & -8.24 & 13.26 & -0.82 & 20.71 & 6.62 \\
\hline $\left.\mathrm{Ti}\left(\mathrm{N}\left(\mathrm{CH}_{3}\right)_{2}\right)_{3}{ }^{---} \mathrm{SHRRSH}^{\star}+\mathrm{HN}\left(\mathrm{CH}_{3}\right)_{2}\right)_{4}$ & -6.40 & -12.91 & -7.21 & -6.13 & -4.94 & 32.79 & 33.87 & 35.06 \\
\hline RS---H---Ti(N( $\left.\left(\mathrm{CH}_{3}\right)_{2}\right)_{3} \mathrm{SR}$ & 12.76 & 0.81 & 8.92 & 11.29 & 13.90 & 48.92 & 51.29 & 53.90 \\
\hline$\left.\left(\mathrm{CH}_{3}\right)_{2}\right) \mathrm{NH}---\mathrm{RS}---\mathrm{Ti}\left(\mathrm{N}\left(\mathrm{CH}_{3}\right)_{2}\right)_{2} \mathrm{SR}$ & -12.19 & -35.30 & -1.40 & 0.72 & 2.96 & 38.60 & 40.72 & 42.96 \\
\hline$\left.\left(\mathrm{CH}_{3}\right)_{2}\right) \mathrm{NH}+\mathrm{RSTi}\left(\mathrm{N}\left(\mathrm{CH}_{3}\right)_{2}\right)_{2} \mathrm{SR}$ & -4.87 & -16.22 & -16.51 & -20.17 & -23.60 & 23.49 & 19.83 & 16.40 \\
\hline $\mathrm{RSTi}\left(\mathrm{N}\left(\mathrm{CH}_{3}\right)_{2}\right)-\mathrm{CH}_{2}---\mathrm{H}-\mathrm{N}\left(\mathrm{CH}_{3}\right)_{2} \mathrm{RS}$ & 35.61 & 24.45 & 20.46 & 16.63 & 13.06 & 60.46 & 56.63 & 53.06 \\
\hline $\mathrm{RSTi}\left(\mathrm{N}\left(\mathrm{CH}_{3}\right)_{2}\right)-\mathrm{CH}_{2}-\mathrm{SR}---\mathrm{HN}\left(\mathrm{CH}_{3}\right)_{2}$ & 18.25 & -0.16 & 0.50 & -2.64 & -5.47 & 40.50 & 37.36 & 34.53 \\
\hline $\mathrm{RSTi}\left(\mathrm{N}\left(\mathrm{CH}_{3}\right)_{2}\right)-\mathrm{CH}_{2}-\mathrm{SR}+\mathrm{HN}\left(\mathrm{CH}_{3}\right)_{2}$ & 44.57 & 41.29 & 30.23 & 21.47 & 13.11 & 70.23 & 61.47 & 53.11 \\
\hline
\end{tabular}


Figure S1

\begin{tabular}{|c|c|c|c|c|}
\hline & Uncatalyzed & Catalyzed & $\begin{array}{l}\text { catalyzed-second } \\
\text { reaction }\end{array}$ & \\
\hline $\mathrm{Ti}\left(\mathrm{N}\left(\mathrm{CH}_{3}\right)_{2}\right)_{4}+\mathrm{NH}_{3}$ & 0.00 & 0.00 & & $\mathrm{Ti}\left(\mathrm{N}\left(\mathrm{CH}_{3}\right)_{2}\right)_{4}+2 \mathrm{NH}_{3}$ \\
\hline $\mathrm{Ti}\left(\mathrm{N}\left(\mathrm{CH}_{3}\right)_{2}\right)_{4^{---} \mathrm{NH}_{3}}$ & -2.77 & -2.28 & & $\mathrm{NH}_{3}---\mathrm{Ti}\left(\mathrm{N}\left(\mathrm{CH}_{3}\right)_{2}\right)_{4}{ }^{---} \mathrm{NH}_{3}$ \\
\hline $\mathrm{Ti}\left(\mathrm{N}\left(\mathrm{CH}_{3}\right)_{2}\right)_{3}{ }^{---} \mathrm{N}(\mathrm{CH} 3)_{2}--\mathrm{H}--\mathrm{NH}_{2}$ & 16.16 & 9.79 & & $\mathrm{NH}_{3}---\mathrm{Ti}\left(\mathrm{N}\left(\mathrm{CH}_{3}\right)_{2}\right)_{3}---\mathrm{N}(\mathrm{CH} 3)_{2}--\mathrm{H}--\mathrm{NH}_{2}$ \\
\hline $\mathrm{Ti}\left(\mathrm{N}\left(\mathrm{CH}_{3}\right)_{2}\right)_{3} \mathrm{NH}_{2}---\mathrm{HN}\left(\mathrm{CH}_{3}\right)_{2}$ & -4.20 & -11.02 & -11.02 & $\mathrm{NH}_{3}---\mathrm{Ti}\left(\mathrm{N}\left(\mathrm{CH}_{3}\right)_{2}\right)_{3} \mathrm{NH}_{2}--\mathrm{HN}\left(\mathrm{CH}_{3}\right)_{2}$ \\
\hline \multirow[t]{3}{*}{$\mathrm{Ti}\left(\mathrm{N}\left(\mathrm{CH}_{3}\right)_{2}\right)_{3} \mathrm{NH}_{2}+\mathrm{HN}\left(\mathrm{CH}_{3}\right)_{2}$} & -0.42 & -0.42 & 9.26 & $\mathrm{HN}\left(\mathrm{CH}_{3}\right)_{2}$ \\
\hline & & & -9.31 & $\mathrm{HN}\left(\mathrm{CH}_{3}\right)_{2}--\mathrm{NH}_{2} \mathrm{Ti}\left(\mathrm{N}\left(\mathrm{CH}_{3}\right)_{2}\right) 2 \mathrm{NH}_{2}---\mathrm{HN}\left(\mathrm{CH}_{3}\right)_{2}$ \\
\hline & & & 0.10 & $\mathrm{NH}_{2} \mathrm{Ti}\left(\mathrm{N}\left(\mathrm{CH}_{3}\right)_{2}\right)_{2} \mathrm{NH}_{2}+2 \mathrm{HN}\left(\mathrm{CH}_{3}\right)_{2}$ \\
\hline
\end{tabular}




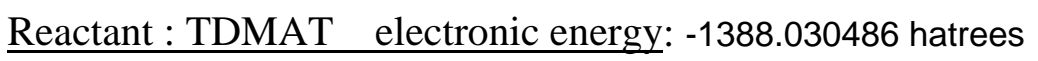

$\begin{array}{llll}\mathrm{Ti} & -0.091175 & -0.153290 & -0.245716 \\ \mathrm{n} & -0.033854 & -0.255019 & 1.671055 \\ \mathrm{~h} & 1.663527 & 0.262104 & -0.905679 \\ \mathrm{n} & -0.672336 & -1.835217 & -0.968056 \\ \mathrm{n} & -1.316602 & 1.221864 & -0.786377 \\ \mathrm{c} & -0.937257 & 0.354253 & 2.629167 \\ \mathrm{c} & 1.059060 & -0.963565 & 2.318253 \\ \mathrm{c} & 2.370035 & -0.355533 & -2.012894 \\ \mathrm{c} & 2.467306 & 1.274290 & -0.238458 \\ \mathrm{c} & -1.138703 & -1.913414 & -2.343326 \\ \mathrm{c} & -0.759439 & -3.113161 & -0.285927 \\ \mathrm{c} & -1.011312 & 2.529651 & -1.335798 \\ \mathrm{c} & -2.748277 & 0.978641 & -0.705666 \\ \mathrm{~h} & 3.414600 & 0.859154 & 0.140366 \\ \mathrm{~h} & 2.724308 & 2.104279 & -0.915210 \\ \mathrm{~h} & 1.928331 & 1.696430 & 0.613754 \\ \mathrm{~h} & 3.303181 & -0.838561 & -1.680699 \\ \mathrm{~h} & 1.752011 & -1.120254 & -2.485878 \\ \mathrm{~h} & 2.646302 & 0.382166 & -2.782837 \\ \mathrm{~h} & 1.643514 & -0.300140 & 2.975050 \\ \mathrm{~h} & 0.696050 & -1.797923 & 2.938714 \\ \mathrm{~h} & 1.745473 & -1.377743 & 1.574721 \\ \mathrm{~h} & -0.414937 & 1.074823 & 3.279152 \\ \mathrm{~h} & -1.736578 & 0.891184 & 2.116258 \\ \mathrm{~h} & -1.400741 & -0.396684 & 3.288434 \\ \mathrm{~h} & -1.792403 & -3.496996 & -0.272564 \\ \mathrm{~h} & -0.137418 & -3.880182 & -0.773968 \\ \mathrm{~h} & -0.428363 & -3.019352 & 0.749375 \\ \mathrm{~h} & -2.179548 & -2.268948 & -2.401571 \\ \mathrm{~h} & -1.098991 & -0.931157 & -2.821641 \\ \mathrm{~h} & -0.526060 & -2.602866 & -2.945356 \\ \mathrm{~h} & -1.422897 & 3.340110 & -0.713601 \\ \mathrm{~h} & 0.067751 & 2.673353 & -1.406672 \\ \mathrm{~h} & -1.432208 & 2.654632 & -2.346647 \\ \mathrm{~h} & -3.249137 & 1.696690 & -0.037452 \\ \mathrm{~h} & -3.232760 & 1.059733 & -1.691490 \\ \mathrm{~h} & -2.949097 & -0.025536 & -0.322528\end{array}$

Amine Surface

$-2012.172394$ 


$\begin{array}{llll}\text { c } & -2.992957 & 1.073116 & 0.212549 \\ \mathrm{c} & -2.018355 & 2.143481 & -0.283096 \\ \mathrm{~h} & -2.704057 & 3.437809 & -0.391325 \\ \mathrm{Si} & -2.247500 & -0.680253 & 0.566387 \\ \mathrm{Si} & -1.353272 & -1.948749 & -1.233416 \\ \mathrm{Si} & 0.451509 & -0.762815 & -2.202576 \\ \mathrm{Si} & 2.218565 & -0.737924 & -0.575749 \\ \mathrm{c} & 3.031351 & 0.981658 & -0.205820 \\ \mathrm{c} & 2.091493 & 2.089905 & 0.274367 \\ \mathrm{~h} & 2.826851 & 3.354944 & 0.399416 \\ \mathrm{Si} & -0.488149 & -0.760240 & 2.214077 \\ \mathrm{Si} & 1.298830 & -1.978704 & 1.232630 \\ \mathrm{~h} & 0.459295 & -2.979437 & 0.461736 \\ \mathrm{~h} & -3.331676 & -1.519135 & 1.164629 \\ \mathrm{~h} & -1.046196 & -1.570663 & 3.338095 \\ \mathrm{~h} & 3.251885 & -1.612762 & -1.213502 \\ \mathrm{~h} & 0.972785 & -1.596034 & -3.324613 \\ \mathrm{~h} & -0.101905 & 0.593553 & 2.745952 \\ \mathrm{~h} & 2.197440 & -2.814024 & 2.078090 \\ \mathrm{~h} & -2.232628 & -2.724289 & -2.151505 \\ \mathrm{~h} & -3.802871 & 0.936256 & -0.517773 \\ \mathrm{~h} & -3.465291 & 1.424802 & 1.137933 \\ \mathrm{~h} & 3.818883 & 0.810963 & 0.541611 \\ \mathrm{~h} & 3.537218 & 1.315625 & -1.119808 \\ \mathrm{~h} & -1.206245 & 2.265822 & 0.444672 \\ \mathrm{~h} & -1.549923 & 1.809625 & -1.226105 \\ \mathrm{~h} & 1.298514 & 2.246838 & -0.467528 \\ \mathrm{~h} & 1.592738 & 1.772900 & 1.207965 \\ \mathrm{~h} & 0.084541 & 0.592889 & -2.741781 \\ \mathrm{~h} & -0.573131 & -2.984373 & -0.437890 \\ \mathrm{~h} & -2.045223 & 4.154898 & -0.692716 \\ \mathrm{~h} & -3.414824 & 3.385796 & -1.120723 \\ \mathrm{~h} & 2.190882 & 4.098975 & 0.684025 \\ \mathrm{~h} & 3.517234 & 3.275128 & 1.145649\end{array}$

$\underline{\mathrm{Ti}\left(\mathrm{N}\left(\mathrm{CH}_{3}\right)_{2}\right)_{4}-{ }_{--2} \mathrm{RNH}_{2}{ }_{2}{ }^{*} \quad-3400.157298}$

$\begin{array}{llll}\text { c } & 2.741439 & -3.067856 & 0.299410 \\ \text { c } & 1.554906 & -2.343398 & -0.336676 \\ \text { n } & 0.324423 & -3.129822 & -0.182564 \\ \text { Si } & 4.375366 & -2.093560 & 0.466362 \\ \text { Si } & 5.269055 & -0.995077 & -1.420471 \\ \text { Si } & 3.737286 & 0.613851 & -2.192932 \\ \text { Si } & 3.614874 & 2.319822 & -0.532774 \\ \text { c } & 1.856244 & 2.826991 & 0.011364\end{array}$




\begin{tabular}{|c|c|c|c|}
\hline C & 0.956773 & 1.738888 & 0.600897 \\
\hline $\mathrm{n}$ & -0.404405 & 2.250188 & 0.799214 \\
\hline Si & 4.365146 & -0.376038 & 2.135719 \\
\hline $\mathrm{Si}$ & 5.159447 & 1.588040 & 1.096142 \\
\hline $\mathrm{n}$ & -3.045614 & -0.322190 & 1.724709 \\
\hline C & -3.838222 & 0.113553 & 2.867739 \\
\hline $\mathrm{Ti}$ & -3.853986 & 0.003458 & 0.008542 \\
\hline $\mathrm{n}$ & -5.570708 & -0.859730 & -0.029080 \\
\hline C & -5.784551 & -2.141715 & 0.623718 \\
\hline $\mathrm{n}$ & -4.063860 & 1.877010 & -0.331205 \\
\hline C & -3.863378 & 3.004854 & 0.561405 \\
\hline $\mathrm{n}$ & -2.782592 & -0.781902 & -1.388515 \\
\hline C & -1.676641 & -0.025538 & -1.962388 \\
\hline C & -4.334804 & 2.309075 & -1.694490 \\
\hline C & -3.107917 & -1.951896 & -2.185367 \\
\hline C & -1.913295 & -1.134847 & 2.132916 \\
\hline C & -6.794414 & -0.342577 & -0.615090 \\
\hline h & 6.169408 & 0.926240 & 0.206952 \\
\hline h & 5.399100 & -3.056087 & 0.945715 \\
\hline h & 5.336049 & -0.820430 & 3.169940 \\
\hline h & 4.211260 & 3.491939 & -1.226750 \\
\hline h & 4.314318 & 1.267204 & -3.392408 \\
\hline h & 3.032682 & -0.225120 & 2.791784 \\
\hline h & 5.916604 & 2.594859 & 1.883998 \\
\hline h & 6.046777 & -1.736820 & -2.444884 \\
\hline h & 2.966140 & -3.981339 & -0.266654 \\
\hline h & 2.451323 & -3.399575 & 1.302809 \\
\hline h & 1.959542 & 3.653037 & 0.727295 \\
\hline h & 1.356146 & 3.253127 & -0.865539 \\
\hline h & 1.387764 & -1.388539 & 0.172126 \\
\hline h & 1.791186 & -2.102532 & -1.384359 \\
\hline h & 0.884812 & 0.902655 & -0.101031 \\
\hline h & 1.409978 & 1.339272 & 1.521410 \\
\hline h & 2.406963 & 0.053067 & -2.570190 \\
\hline h & 6.234811 & -0.080278 & -0.719171 \\
\hline h & -0.461393 & -2.639894 & -0.600513 \\
\hline h & 0.411125 & -4.015741 & -0.672799 \\
\hline h & -1.029144 & 1.514295 & 1.118486 \\
\hline h & -0.408968 & 2.970886 & 1.515039 \\
\hline h & -5.267287 & 2.890931 & -1.758434 \\
\hline h & -3.525321 & 2.941758 & -2.087807 \\
\hline h & -4.436336 & 1.450086 & -2.363960 \\
\hline h & -4.743305 & 3.666450 & 0.578363 \\
\hline h & -3.684265 & 2.664647 & 1.583104 \\
\hline h & -2.996155 & 3.607021 & 0.254880 \\
\hline h & -3.392606 & -1.679500 & -3.214787 \\
\hline
\end{tabular}




$\begin{array}{llll}\text { h } & -2.251877 & -2.641547 & -2.267142 \\ \text { h } & -3.939972 & -2.500768 & -1.743856 \\ \text { h } & -1.892544 & 0.297926 & -2.993070 \\ \text { h } & -1.461064 & 0.867762 & -1.371517 \\ \text { h } & -0.755612 & -0.628191 & -2.006207 \\ \text { h } & -2.235100 & -2.014712 & 2.711946 \\ \text { h } & -1.358181 & -1.502107 & 1.271185 \\ \text { h } & -1.217685 & -0.571934 & 2.775739 \\ \text { h } & -4.172575 & -0.738364 & 3.479053 \\ \text { h } & -3.271450 & 0.788494 & 3.527357 \\ \text { h } & -4.735463 & 0.650467 & 2.543392 \\ \text { h } & -7.583750 & -0.211558 & 0.142447 \\ \text { h } & -6.622438 & 0.628653 & -1.081226 \\ \text { h } & -7.195163 & -1.021540 & -1.384211 \\ \text { h } & -4.861471 & -2.502899 & 1.083098 \\ \text { h } & -6.548050 & -2.074928 & 1.414675 \\ \text { h } & -6.125123 & -2.909071 & -0.089389\end{array}$

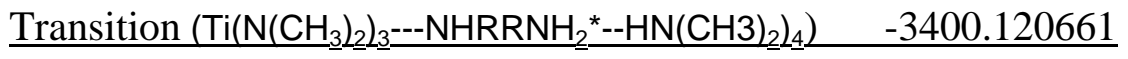

$\begin{array}{llll}\text { c } & -3.161902 & 3.041647 & -0.067234 \\ \mathrm{c} & -1.914683 & 2.568839 & -0.813981 \\ \mathrm{n} & -0.911379 & 3.640166 & -0.873074 \\ \mathrm{Si} & -4.460742 & 1.719607 & 0.391323 \\ \mathrm{Si} & -5.202002 & 0.202160 & -1.254833 \\ \mathrm{Si} & -3.376966 & -1.057199 & -2.043559 \\ \mathrm{Si} & -2.655481 & -2.450509 & -0.247603 \\ \mathrm{c} & -0.782636 & -2.430496 & 0.139714 \\ \mathrm{c} & -0.156214 & -1.095493 & 0.566480 \\ \mathrm{n} & 1.290258 & -1.178087 & 0.805705 \\ \mathrm{Si} & -3.844280 & 0.288540 & 2.209441 \\ \mathrm{Si} & -4.184280 & -1.927109 & 1.474230 \\ \mathrm{n} & 2.374099 & 0.879897 & 1.740638 \\ \mathrm{c} & 3.161327 & 0.922512 & 2.965805 \\ \mathrm{Ti} & 3.146601 & -0.161421 & -0.039339 \\ \mathrm{n} & 4.736852 & 0.970609 & -0.068807 \\ \mathrm{c} & 4.846280 & 2.363956 & 0.325908 \\ \mathrm{n} & 4.027157 & -1.862720 & -0.012806 \\ \mathrm{c} & 4.901296 & -2.217926 & 1.092577 \\ \mathrm{n} & 2.363808 & 0.309236 & -1.729982 \\ \mathrm{c} & 1.445139 & -0.565016 & -2.445110 \\ \mathrm{c} & 3.750384 & -2.994772 & -0.875466 \\ \mathrm{c} & 2.732966 & 1.444137 & -2.561247 \\ \mathrm{c} & 1.496735 & 2.041072 & 1.610137 \\ \mathrm{c} & 6.007703 & 0.530206 & -0.632898 \\ \mathrm{~h} & -5.410777 & -1.660979 & 0.652069\end{array}$




\begin{tabular}{|c|c|c|c|}
\hline h & -5.654595 & 2.447889 & 0.891503 \\
\hline h & -4.800325 & 0.602861 & 3.303053 \\
\hline h & -2.984625 & -3.812821 & -0.746222 \\
\hline h & -3.869354 & -1.981017 & -3.093987 \\
\hline h & -2.467770 & 0.566620 & 2.714332 \\
\hline h & -4.574122 & -2.984004 & 2.443751 \\
\hline h & -6.238892 & 0.586326 & -2.246646 \\
\hline h & -3.677530 & 3.812117 & -0.655212 \\
\hline h & -2.847800 & 3.531529 & 0.861363 \\
\hline h & -0.613950 & -3.176370 & 0.927964 \\
\hline h & -0.268685 & -2.817223 & -0.748016 \\
\hline h & -1.454495 & 1.731930 & -0.278909 \\
\hline h & -2.203623 & 2.185939 & -1.804560 \\
\hline h & -0.334545 & -0.342923 & -0.209375 \\
\hline h & -0.685822 & -0.735799 & 1.462267 \\
\hline $\mathrm{h}$ & -2.281369 & -0.234477 & -2.634490 \\
\hline h & -5.823914 & -0.830098 & -0.354875 \\
\hline h & -0.087009 & 3.317652 & -1.371827 \\
\hline h & -1.278112 & 4.429692 & -1.397699 \\
\hline h & 1.680498 & -0.183976 & 1.586785 \\
\hline h & 1.513357 & -2.081411 & 1.214403 \\
\hline h & 4.681420 & -3.433253 & -1.265386 \\
\hline h & 3.211045 & -3.795482 & -0.343875 \\
\hline h & 3.145260 & -2.696251 & -1.732026 \\
\hline h & 5.857102 & -2.627699 & 0.732446 \\
\hline h & 5.134269 & -1.340507 & 1.700297 \\
\hline h & 4.450098 & -2.978353 & 1.749630 \\
\hline h & 3.224147 & 1.122353 & -3.493165 \\
\hline h & 1.843642 & 2.024496 & -2.857341 \\
\hline h & 3.413107 & 2.109319 & -2.034260 \\
\hline h & 1.895892 & -0.944964 & -3.375682 \\
\hline h & 1.165186 & -1.417639 & -1.829060 \\
\hline & 0.522566 & -0.036936 & -2.730587 \\
\hline h & 2.050331 & 2.986884 & 1.542746 \\
\hline h & 0.888443 & 1.959421 & 0.708183 \\
\hline h & 0.816618 & 2.130345 & 2.469846 \\
\hline h & 3.903764 & 1.730492 & 2.961041 \\
\hline n & 2.519028 & 1.069938 & 3.846683 \\
\hline h & 3.698774 & -0.019356 & 3.109361 \\
\hline & 6.833043 & 0.651929 & 0.087342 \\
\hline & 5.960050 & -0.515668 & -0.928980 \\
\hline h & 6.274528 & 1.122573 & -1.522571 \\
\hline 11 & 3.905908 & 2.743310 & 0.719068 \\
\hline h & 5.619981 & 2.510266 & 1.096668 \\
\hline h & 5.132584 & 3.002025 & -0.526373 \\
\hline
\end{tabular}




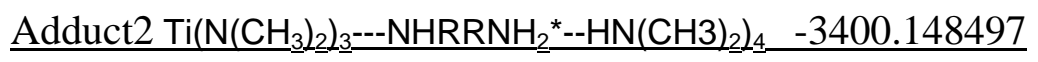

$\begin{array}{llll}\text { c } & 2.545274 & 3.015444 & 0.110523 \\ \mathrm{Si} & 3.963492 & 1.909847 & -0.538484 \\ \mathrm{Si} & 3.205817 & 0.358549 & -2.198164 \\ \mathrm{Si} & 3.929978 & -1.794564 & -1.549078 \\ \mathrm{Si} & 2.862782 & -2.461202 & 0.454195 \\ \mathrm{c} & 0.957296 & -2.613274 & 0.480919 \\ \mathrm{c} & 0.175237 & -1.559060 & -0.322247 \\ \mathrm{n} & -1.238884 & -1.507099 & 0.003626 \\ \mathrm{Ti} & -2.811930 & -0.256925 & 0.074609 \\ \mathrm{n} & -4.175186 & 1.134591 & -0.393935 \\ \mathrm{C} & -5.277423 & 0.929491 & -1.317070 \\ \mathrm{c} & 1.498631 & 2.332375 & 0.996122 \\ \mathrm{n} & 0.251331 & 3.107350 & 1.037937 \\ \mathrm{Si} & 5.176078 & 0.551513 & 0.965890 \\ \mathrm{Si} & 3.723010 & -0.934019 & 2.071016 \\ \mathrm{n} & -4.061581 & -1.734464 & -0.018056 \\ \mathrm{c} & -5.294448 & -1.765200 & 0.751652 \\ \mathrm{n} & -2.563217 & 0.378354 & 1.900272 \\ \mathrm{c} & -3.645995 & 0.850056 & 2.753449 \\ \mathrm{n} & -1.565675 & 0.628879 & -1.703197 \\ \mathrm{c} & -1.493064 & 2.088948 & -1.916665 \\ \mathrm{c} & -3.902316 & -2.964098 & -0.774555 \\ \mathrm{c} & -1.390937 & 0.110110 & 2.721375 \\ \mathrm{c} & -2.052092 & -0.061410 & -2.915142 \\ \mathrm{c} & -4.138430 & 2.528153 & 0.013380 \\ \mathrm{~h} & 5.253623 & -1.371858 & -1.012606 \\ \mathrm{~h} & 4.931857 & 2.785626 & -1.254641 \\ \mathrm{~h} & 2.650738 & -0.254639 & 2.855437 \\ \mathrm{~h} & 3.860647 & 0.766355 & -3.470493 \\ \mathrm{~h} & 3.454036 & -3.755030 & 0.895960 \\ \mathrm{~h} & 4.511913 & -1.770688 & 3.010430 \\ \mathrm{~h} & 1.730226 & 0.469937 & -2.411224 \\ \mathrm{~h} & 4.257309 & -2.814681 & -2.581340 \\ \mathrm{~h} & 6.322686 & 1.091152 & 1.743159 \\ \mathrm{~h} & 2.982147 & 3.875446 & 0.632455 \\ \mathrm{~h} & 2.038590 & 3.423315 & -0.771727 \\ \mathrm{~h} & 0.678322 & -3.616196 & 0.137471 \\ \mathrm{~h} & 0.650392 & -2.557233 & 1.532023 \\ \mathrm{~h} & 1.239551 & 1.351305 & 0.585730 \\ \mathrm{~h} & 1.928050 & 2.140170 & 1.990555 \\ \mathrm{~h} & 0.632370 & -0.573410 & -0.136863 \\ \mathrm{~h} & 0.353751 & -1.761642 & -1.394587 \\ \mathrm{~h} & -0.3949094 & 2.613043 & 1.583641\end{array}$




$\begin{array}{llll}\text { h } & 0.404873 & 4.003893 & 1.490285 \\ \text { h } & -0.619361 & 0.300481 & -1.541006 \\ \text { h } & -1.506638 & -2.378739 & 0.453367 \\ \text { h } & -6.171909 & -1.922020 & 0.103842 \\ \text { h } & -5.289030 & -2.582881 & 1.489496 \\ \text { h } & -5.445550 & -0.829401 & 1.287820 \\ \text { h } & -4.718878 & -3.100958 & -1.502011 \\ \text { h } & -2.958309 & -2.964964 & -1.318227 \\ \text { h } & -3.913966 & -3.851446 & -0.118713 \\ \text { h } & -3.901673 & 0.107887 & 3.526907 \\ \text { h } & -3.368431 & 1.775532 & 3.283622 \\ \text { h } & -4.540974 & 1.055098 & 2.170781 \\ \text { h } & -1.585687 & -0.672769 & 3.473701 \\ \text { h } & -0.554595 & -0.219755 & 2.110028 \\ \text { h } & -1.081522 & 1.008023 & 3.283568 \\ \text { h } & -2.493087 & 2.471067 & -2.105555 \\ \text { h } & -1.083505 & 2.575199 & -1.030192 \\ \text { h } & -0.856553 & 2.327842 & -2.778609 \\ \text { h } & -3.090327 & 0.217743 & -3.095267 \\ \text { h } & -1.461646 & 0.207171 & -3.799611 \\ \text { h } & -1.996606 & -1.139096 & -2.763562 \\ \text { h } & -5.179260 & 1.535267 & -2.237020 \\ \text { h } & -5.346361 & -0.118448 & -1.610255 \\ \text { h } & -6.243056 & 1.217694 & -0.867225 \\ \text { h } & -3.277364 & 2.727358 & 0.652485 \\ \text { h } & -4.090251 & 3.223892 & -0.841911 \\ \text { h } & -5.045384 & 2.807569 & 0.576702\end{array}$

Product $\quad \mathrm{Ti}\left(\mathrm{N}(\mathrm{CH} 3)_{2}\right)_{3}---\mathrm{NHRRNH}_{2} \underline{2}^{*}-3264.959026$

$\begin{array}{llll}\text { c } & -0.489972 & -2.472978 & -0.061583 \\ \mathrm{c} & 0.463835 & -1.656311 & -0.955561 \\ \mathrm{n} & 1.859401 & -1.709156 & -0.540967 \\ \mathrm{Ti} & 2.994997 & -0.251183 & -0.011541 \\ \mathrm{n} & 3.165499 & 1.072478 & -1.412490 \\ \mathrm{Si} & -2.348665 & -2.413498 & -0.549151 \\ \mathrm{Si} & -3.834899 & -1.935805 & 1.210852 \\ \mathrm{Si} & -3.255857 & 0.125287 & 2.174543 \\ \mathrm{Si} & -3.717015 & 1.808973 & 0.557311 \\ \mathrm{c} & -2.239025 & 2.978253 & 0.247384 \\ \mathrm{c} & -0.951113 & 2.294229 & -0.220808 \\ \mathrm{n} & 0.179678 & 3.229394 & -0.227207 \\ \mathrm{Si} & -2.923858 & -0.773305 & -2.205246 \\ \mathrm{Si} & -4.601871 & 0.581791 & -1.255771 \\ \mathrm{n} & 4.703558 & -1.034366 & 0.325525 \\ \mathrm{n} & 2.426530 & 0.537279 & 1.640726\end{array}$




\begin{tabular}{|c|c|c|c|}
\hline h & -5.303728 & -0.504432 & -0.499167 \\
\hline h & -2.786293 & -3.701801 & -1.161398 \\
\hline h & -3.478407 & -1.537997 & -3.349532 \\
\hline h & -4.838404 & 2.570522 & 1.174663 \\
\hline h & -4.180915 & 0.401977 & 3.299637 \\
\hline h & -1.747752 & -0.013335 & -2.718306 \\
\hline h & -5.635076 & 1.201272 & -2.124428 \\
\hline h & -4.303428 & -2.996319 & 2.133472 \\
\hline h & -0.382021 & -2.129392 & 0.971125 \\
\hline h & -0.183433 & -3.525647 & -0.064552 \\
\hline h & -2.542599 & 3.745597 & -0.476014 \\
\hline h & -2.032778 & 3.507846 & 1.184053 \\
\hline h & 0.357114 & -1.986769 & -2.000148 \\
\hline h & 0.144775 & -0.608530 & -0.948062 \\
\hline h & -0.687120 & 1.491727 & 0.475699 \\
\hline h & -1.124200 & 1.818729 & -1.198744 \\
\hline h & -1.856141 & 0.176410 & 2.692101 \\
\hline h & -5.017030 & -1.490429 & 0.406852 \\
\hline h & 1.036265 & 2.727393 & -0.447086 \\
\hline h & 0.052082 & 3.931583 & -0.950331 \\
\hline C & 1.731173 & -0.211911 & 2.677466 \\
\hline C & 2.633206 & 1.926463 & 2.028398 \\
\hline C & 5.460074 & -1.522774 & -0.818622 \\
\hline C & 5.425994 & -1.190400 & 1.573954 \\
\hline C & 2.431304 & 1.139036 & -2.664350 \\
\hline C & 4.288496 & 1.998412 & -1.394803 \\
\hline h & 2.225474 & -2.654765 & -0.590432 \\
\hline h & 2.301641 & -0.217915 & 3.619130 \\
\hline h & 0.743862 & 0.220425 & 2.900712 \\
\hline h & 1.585235 & -1.247967 & 2.368651 \\
\hline h & 3.185804 & 1.994681 & 2.977650 \\
\hline h & 3.209115 & 2.462395 & 1.271061 \\
\hline h & 1.683207 & 2.463985 & 2.151960 \\
\hline h & 6.422081 & -0.998922 & -0.928690 \\
\hline h & 5.680553 & -2.597706 & -0.728604 \\
\hline h & 4.896922 & -1.379222 & -1.744786 \\
\hline h & 6.383328 & -0.645148 & 1.560083 \\
\hline h & 4.833084 & -0.809296 & 2.405415 \\
\hline h & 5.661334 & -2.246916 & 1.777047 \\
\hline h & 2.014450 & 2.143356 & -2.841274 \\
\hline h & 3.074391 & 0.903085 & -3.527198 \\
\hline h & 1.601536 & 0.430095 & -2.669701 \\
\hline h & 3.956991 & 3.047515 & -1.438615 \\
\hline h & 4.879578 & 1.875800 & -0.483392 \\
\hline h & 4.964397 & 1.834421 & -2.248433 \\
\hline
\end{tabular}


Transition $\left.\quad \mathrm{RNH}---\mathrm{H}---\mathrm{Ti}\left(\mathrm{N}_{(}\left(\mathrm{CH}_{3}\right)_{2}\right)_{2}\right)_{3} \mathrm{NHR} \quad-3264.915857$

$\begin{array}{llll}\text { c } & 0.825632 & -0.688360 & -2.450052 \\ \mathrm{c} & -0.284291 & -1.423778 & -1.660221 \\ \mathrm{n} & -1.565491 & -0.731475 & -1.598325 \\ \mathrm{Ti} & -2.443484 & 0.091260 & -0.099327 \\ \mathrm{n} & -2.536574 & -1.507778 & 1.434119 \\ \mathrm{Si} & 2.633061 & -1.194292 & -2.007837 \\ \mathrm{Si} & 4.084121 & 0.643744 & -1.762123 \\ \mathrm{Si} & 3.127571 & 2.181189 & -0.277228 \\ \mathrm{Si} & 3.095312 & 1.137401 & 1.864231 \\ \mathrm{c} & 1.295744 & 0.994062 & 2.528295 \\ \mathrm{c} & 0.306759 & 0.807425 & 1.354224 \\ \mathrm{n} & -1.059260 & 0.425067 & 1.697887 \\ \mathrm{Si} & 2.757244 & -2.274890 & 0.129516 \\ \mathrm{Si} & 4.136015 & -0.940640 & 1.477090 \\ \mathrm{n} & -4.283710 & -0.100486 & -0.656074 \\ \mathrm{n} & -2.424152 & 2.014963 & -0.326426 \\ \mathrm{~h} & 5.092979 & -0.542491 & 0.406153 \\ \mathrm{~h} & 3.311608 & -2.151468 & -2.946027 \\ \mathrm{~h} & 3.376883 & -3.589154 & -0.139285 \\ \mathrm{~h} & 4.057833 & 1.966321 & 2.666221 \\ \mathrm{~h} & 4.023583 & 3.342644 & -0.118551 \\ \mathrm{~h} & 1.427818 & -2.550634 & 0.745558 \\ \mathrm{~h} & 4.941781 & -1.582815 & 2.540528 \\ \mathrm{~h} & 4.826627 & 1.218793 & -2.905617 \\ \mathrm{~h} & 0.738997 & 0.380723 & -2.232332 \\ \mathrm{~h} & 0.661556 & -0.789697 & -3.528472 \\ \mathrm{~h} & 1.222745 & 0.146052 & 3.216693 \\ \mathrm{~h} & 1.031033 & 1.889981 & 3.101469 \\ \mathrm{~h} & -0.412006 & -2.435642 & -2.071302 \\ \mathrm{~h} & 0.051196 & -1.575236 & -0.633744 \\ \mathrm{~h} & 0.296120 & 1.729251 & 0.762369 \\ \mathrm{~h} & 0.697727 & 0.033834 & 0.682399 \\ \mathrm{~h} & 1.806150 & 2.702799 & -0.728783 \\ \mathrm{~h} & 5.085776 & 0.025983 & -0.839657 \\ \mathrm{~h} & -1.544554 & -0.758269 & 1.835451 \\ \mathrm{~h} & -1.410968 & 1.033914 & 2.431813 \\ \mathrm{c} & -2.384437 & 2.599372 & -1.659102 \\ \mathrm{c} & -2.539437 & 3.048292 & 0.682537 \\ \mathrm{c} & -4.809837 & -1.415631 & -0.994329 \\ \mathrm{~h} & -5.345199 & 0.889581 & -0.666450 \\ \mathrm{~h} & -2.265767 & -2.910577 & 1.146011 \\ \mathrm{~h} & -3.638008 & -1.365808 & 2.382118 \\ \mathrm{~h} & -3.299447 & 3.171769 & -1.881377\end{array}$




$\begin{array}{llll}\text { h } & -1.535445 & 3.292581 & -1.767044 \\ \mathrm{~h} & -2.279909 & 1.826948 & -2.419886 \\ \mathrm{~h} & -3.408473 & 3.699416 & 0.496498 \\ \mathrm{~h} & -2.666157 & 2.620642 & 1.678266 \\ \mathrm{~h} & -1.653243 & 3.703092 & 0.707731 \\ \mathrm{~h} & -5.564651 & -1.765291 & -0.272390 \\ \mathrm{~h} & -5.298590 & -1.395670 & -1.981474 \\ \mathrm{~h} & -4.013519 & -2.158193 & -1.037622 \\ \mathrm{~h} & -6.132337 & 0.653151 & 0.067959 \\ \mathrm{~h} & -4.950076 & 1.876279 & -0.433132 \\ \mathrm{~h} & -5.837661 & 0.938618 & -1.650856 \\ \mathrm{~h} & -2.049998 & -3.470902 & 2.068206 \\ \mathrm{~h} & -3.113387 & -3.410125 & 0.656386 \\ \mathrm{~h} & -1.399275 & -3.007822 & 0.489547 \\ \mathrm{~h} & -3.441627 & -1.912971 & 3.316381 \\ \mathrm{~h} & -3.784168 & -0.312754 & 2.644860 \\ \mathrm{~h} & -4.587616 & -1.734788 & 1.974755\end{array}$

Adduct2 $\left.\quad\left(\mathrm{CH}_{3}\right)_{2}\right) \mathrm{NH}---\mathrm{RNH}---\mathrm{Ti}\left(\mathrm{N}\left(\mathrm{CH}_{3}\right)_{2} 2_{2}{ }_{2} \mathrm{NHR} \quad-3264.949925\right.$

$\begin{array}{llll}\text { c } & 0.790851 & -0.396839 & 2.639568 \\ \mathrm{Si} & 2.577139 & 0.215071 & 2.247350 \\ \mathrm{Si} & 2.652707 & 2.110713 & 0.779592 \\ \mathrm{Si} & 3.800653 & 1.421178 & -1.154778 \\ \mathrm{Si} & 2.556212 & -0.234960 & -2.288675 \\ \mathrm{c} & 0.709737 & 0.096487 & -2.692734 \\ \mathrm{c} & -0.042423 & 0.592537 & -1.436695 \\ \mathrm{n} & -1.476402 & 0.327399 & -1.367274 \\ \mathrm{c} & -0.381457 & 0.536067 & 2.227050 \\ \mathrm{n} & -1.603360 & -0.163600 & 1.832543 \\ \mathrm{Ti} & -2.217691 & -0.726954 & 0.087597 \\ \mathrm{n} & -2.562559 & 3.411798 & -0.198776 \\ \mathrm{c} & -2.663259 & 4.222968 & -1.402544 \\ \mathrm{n} & -4.115067 & -0.690765 & 0.056436 \\ \mathrm{c} & -5.058127 & -1.786708 & -0.074098 \\ \mathrm{n} & -1.788313 & -2.577899 & -0.155730 \\ \mathrm{c} & -1.729691 & -3.203632 & -1.468709 \\ \mathrm{c} & -4.781993 & 0.579940 & 0.326514 \\ \mathrm{Si} & 3.831640 & -1.405892 & 1.089638 \\ \mathrm{Si} & 2.638117 & -2.084357 & -0.804120 \\ \mathrm{c} & -1.583496 & -3.545096 & 0.910974 \\ \mathrm{C} & -1.932487 & 4.110517 & 0.911729 \\ \mathrm{~h} & 4.796756 & 0.538368 & -0.463378 \\ \mathrm{~h} & 3.412935 & 0.635771 & 3.419271 \\ \mathrm{~h} & 3.424466 & 3.141104 & 1.503061\end{array}$




$\begin{array}{llll}\text { h } & 3.371828 & -0.678761 & -3.456640 \\ \text { h } & 3.395679 & -3.121565 & -1.537406 \\ \text { h } & 1.318990 & 2.713782 & 0.503000 \\ \text { h } & 4.600836 & 2.407481 & -1.922362 \\ \text { h } & 4.589072 & -2.465242 & 1.789565 \\ \text { h } & 0.670489 & -1.332647 & 2.086660 \\ \text { h } & 0.716889 & -0.667856 & 3.698181 \\ \text { h } & 0.586825 & 0.801004 & -3.521802 \\ \text { h } & 0.282712 & -0.856907 & -3.020654 \\ \text { h } & -0.586380 & 1.243552 & 3.040698 \\ \text { h } & -0.073222 & 1.156538 & 1.385143 \\ \text { h } & 0.402263 & 0.114821 & -0.553296 \\ \text { h } & 0.165716 & 1.663758 & -1.314032 \\ \text { h } & 1.273787 & -2.608622 & -0.508621 \\ \text { h } & 4.820191 & -0.512289 & 0.414588 \\ \text { h } & -2.064915 & 2.549239 & -0.401395 \\ \text { h } & -1.988297 & 0.739722 & -2.140292 \\ \text { h } & -2.200573 & -0.361489 & 2.629891 \\ \text { h } & -2.348929 & -4.336299 & 0.889527 \\ \text { h } & -0.603816 & -4.040086 & 0.825038 \\ \text { h } & -1.629423 & -3.056599 & 1.884642 \\ \text { h } & -2.503553 & -3.978986 & -1.579743 \\ \text { h } & -1.883144 & -2.463866 & -2.255737 \\ \text { h } & -0.757044 & -3.688131 & -1.643156 \\ \text { h } & -5.512958 & 0.813659 & -0.461944 \\ \text { h } & -5.327825 & 0.552885 & 1.282544 \\ \text { h } & -4.078653 & 1.415637 & 0.364150 \\ \text { h } & -5.766263 & -1.611705 & -0.898561 \\ \text { h } & -4.532441 & -2.721179 & -0.268246 \\ \text { h } & -5.657167 & -1.914652 & 0.841473 \\ \text { h } & -0.932359 & 4.517376 & 0.679882 \\ \text { h } & -2.563921 & 4.947935 & 1.224846 \\ \text { h } & -1.837026 & 3.432585 & 1.762445 \\ \text { h } & -1.699779 & 4.637418 & -1.750379 \\ \text { h } & -3.092949 & 3.629402 & -2.213557 \\ \text { h } & -3.336161 & 5.067292 & -1.223337 \\ & & & \end{array}$

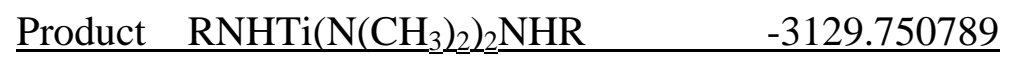

$\begin{array}{llll}\mathrm{c} & 0.556741 & 2.256017 & 1.404735 \\ \mathrm{c} & -0.433217 & 2.436469 & 0.219246 \\ \mathrm{n} & -1.752146 & 1.850921 & 0.445117 \\ \mathrm{Ti} & -2.513183 & 0.169484 & -0.147244 \\ \mathrm{n} & -2.626326 & -1.080659 & 1.300388 \\ \mathrm{c} & -2.733286 & -0.698701 & 2.699638 \\ \mathrm{Si} & 2.422347 & 2.024076 & 0.974438\end{array}$




$\begin{array}{llll}\text { Si } & 3.327540 & 0.080791 & 1.949850 \\ \mathrm{Si} & 2.010752 & -1.758526 & 1.349310 \\ \mathrm{Si} & 2.288420 & -2.091766 & -0.983609 \\ \mathrm{c} & 0.545261 & -2.071389 & -1.781333 \\ \mathrm{c} & -0.106928 & -0.679483 & -1.613342 \\ \mathrm{~h} & -1.562531 & -0.624247 & -1.620065 \\ \mathrm{Si} & 2.857775 & 1.711135 & -1.363214 \\ \mathrm{Si} & 3.838358 & -0.416379 & -1.588166 \\ \mathrm{~h} & -4.309673 & 0.448907 & -0.713716 \\ \mathrm{c} & -4.553669 & 1.513238 & -1.677615 \\ \mathrm{c} & -5.530043 & -0.223280 & -0.309806 \\ \mathrm{c} & -2.637438 & -2.525500 & 1.130399 \\ \mathrm{~h} & 4.646321 & -0.374154 & -0.325564 \\ \mathrm{~h} & 3.331869 & 3.151867 & 1.359363 \\ \mathrm{~h} & 3.822592 & 2.766891 & -1.732523 \\ \mathrm{~h} & 2.992411 & -3.403235 & -1.074441 \\ \mathrm{~h} & 2.548673 & -2.987952 & 1.972196 \\ \mathrm{~h} & 1.665738 & 1.921783 & -2.231766 \\ \mathrm{~h} & 4.811668 & -0.664936 & -2.681130 \\ \mathrm{~h} & 3.867624 & 0.069829 & 3.326457 \\ \mathrm{~h} & 0.257869 & 1.349960 & 1.939494 \\ \mathrm{~h} & 0.448921 & 3.073456 & 2.125956 \\ \mathrm{~h} & 0.566287 & -2.366818 & -2.835519 \\ \mathrm{~h} & -0.056744 & -2.819142 & -1.254623 \\ \mathrm{~h} & -0.517253 & 3.504188 & -0.023441 \\ \mathrm{~h} & -6.249066 & 0.477898 & 0.143443 \\ \mathrm{~h} & -0.024807 & 1.974446 & -0.679328 \\ \mathrm{~h} & 0.214062 & -0.264230 & -0.648291 \\ \mathrm{~h} & 0.312097 & -0.004986 & -2.370787 \\ \mathrm{~h} & 0.571046 & -1.626284 & 1.720124 \\ \mathrm{~h} & 4.468680 & -0.151900 & 1.013979 \\ \mathrm{~h} & -1.962115 & -0.887699 & -2.513964 \\ \mathrm{~h} & -2.334590 & 2.454490 & 1.019827 \\ \mathrm{~h} & -3.673290 & -1.057104 & 3.146865 \\ \mathrm{~h} & -1.908016 & -1.116829 & 3.296501 \\ \mathrm{~h} & -2.700878 & 0.386857 & 2.806971 \\ \mathrm{~h} & -3.571908 & -2.968644 & 1.508550 \\ \mathrm{~h} & -2.544965 & -2.785577 & 0.075365 \\ \mathrm{~h} & -1.808283 & -3.003575 & 1.674548 \\ \mathrm{~h} & -5.004007 & 1.124903 & -2.604016 \\ \mathrm{~h} & -3.620372 & 2.013428 & -1.947416 \\ \mathrm{~h} & -5.309395 & -1.000779 & 0.421400 \\ \mathrm{~h} & & & \\ \mathrm{~h} & & & \\ \mathrm{~h} & & & \\ \mathrm{~h} & & \end{array}$

Transition $\quad \mathrm{RNHTi}\left(\mathrm{N}\left(\mathrm{CH}_{3}\right)_{2}\right)--\mathrm{N}\left(\mathrm{CH}_{3}\right)_{2}--\mathrm{H}_{-}-\mathrm{NR} \quad-3129.699423$ 


$\begin{array}{llll}\text { c } & 0.921762 & 3.169531 & 0.694408 \\ \text { c } & -0.394540 & 2.750926 & 0.015566 \\ \text { n } & -1.195121 & 1.769998 & 0.736606 \\ \text { Ti } & -2.043661 & 0.144708 & 0.158486 \\ \text { n } & -2.786910 & -0.964613 & 1.527176 \\ \text { c } & -3.548629 & -0.220466 & 2.520562 \\ \text { Si } & 2.503347 & 2.091273 & 0.568893 \\ \text { Si } & 2.972766 & 0.273704 & 2.007154 \\ \text { Si } & 1.366507 & -1.416976 & 1.691209 \\ \text { Si } & 1.706501 & -2.315482 & -0.495183 \\ \text { c } & 0.242354 & -2.557135 & -1.700342 \\ \text { c } & -0.500091 & -1.325628 & -2.241356 \\ \text { n } & -1.232154 & -0.615792 & -1.226808 \\ \text { Si } & 2.967873 & 1.176082 & -1.599216 \\ \text { Si } & 3.570009 & -1.087015 & -1.276549 \\ \text { n } & -3.472798 & 0.659063 & -1.299687 \\ \text { c } & -3.745779 & 2.038125 & -1.672347 \\ \text { c } & -4.609769 & -0.223451 & -1.521373 \\ \text { c } & -2.706264 & -2.380138 & 1.824958 \\ \text { h } & 4.316601 & -0.893524 & 0.010072 \\ \text { h } & 3.585537 & 3.092809 & 0.767381 \\ \text { h } & 4.141815 & 1.932991 & -2.112603 \\ \text { h } & 2.177985 & -3.696096 & -0.201767 \\ \text { h } & 1.675660 & -2.525327 & 2.631672 \\ \text { h } & 1.846194 & 1.372245 & -2.565712 \\ \text { h } & 4.536076 & -1.740397 & -2.202442 \\ \text { h } & 3.462203 & 0.499127 & 3.396630 \\ \text { h } & 0.735578 & 3.393680 & 1.752558 \\ \text { h } & 1.239955 & 4.123779 & 0.254449 \\ \text { h } & 0.653939 & -3.126230 & -2.544797 \\ \text { h } & -2.124806 & -2.894807 & 1.059341 \\ \text { h } & -0.479717 & -3.222529 & -1.213761 \\ \text { h } & -0.989358 & 3.665753 & -0.137578 \\ \text { h } & -0.192348 & 2.356139 & -0.983501 \\ \text { h } & 0.214336 & -0.655716 & -2.741989 \\ \text { h } & -1.190442 & -1.663528 & -3.030692 \\ \text { h } & -0.025019 & -0.965075 & 1.967165 \\ \text { h } & 4.119900 & -0.343594 & 1.248677 \\ \text { h } & -2.289334 & 0.032272 & -1.694519 \\ \text { h } & -1.386466 & 2.103814 & 1.680310 \\ \text { h } & -4.581343 & -0.588926 & 2.603841 \\ \text { h } & -3.091693 & -0.273223 & 3.520151 \\ \text { h } & -3.704661 & -2.841630 & 1.859462 \\ \text { h } & -2.565291 & 2.797677\end{array}$




$\begin{array}{llll}\text { h } & -4.097847 & 2.107049 & -2.712619 \\ \text { h } & -4.518323 & 2.495583 & -1.035140 \\ \text { h } & -2.843346 & 2.644551 & -1.584102 \\ \text { h } & -4.969006 & -0.160658 & -2.559817 \\ \text { h } & -4.325118 & -1.259780 & -1.327556 \\ \text { h } & -5.458841 & 0.017738 & -0.864732\end{array}$

Adduct2 $\quad \mathrm{RNHTi}\left(\mathrm{N}\left(\mathrm{CH}_{3}\right)_{2}\right)=\mathrm{NR}-\mathrm{HN}\left(\mathrm{CH}_{3}\right)_{2}$ $-3129.742817$

$\begin{array}{llll}\text { c } & 0.750223 & 3.130928 & 0.900637 \\ \text { Si } & 2.377828 & 2.120597 & 0.803901 \\ \text { Si } & 3.007541 & 1.400037 & -1.395019 \\ \text { Si } & 3.696830 & -0.853690 & -1.213539 \\ \text { Si } & 1.852227 & -2.219789 & -0.643029 \\ \text { c } & 0.479862 & -2.408559 & -1.957630 \\ \text { c } & -0.318893 & -1.155701 & -2.366629 \\ \text { n } & -1.108250 & -0.620663 & -1.301566 \\ \text { Ti } & -2.024627 & -0.004904 & 0.000113 \\ \text { n } & -3.837488 & 0.669978 & -1.106541 \\ \text { c } & -4.948077 & 1.099869 & -0.232730 \\ \text { c } & -0.505158 & 2.716067 & 0.110658 \\ \text { n } & -1.316348 & 1.669357 & 0.714232 \\ \text { h } & -2.779062 & -1.386715 & 1.147207 \\ \text { c } & -2.737075 & -2.832067 & 1.039520 \\ \text { c } & -3.265234 & -0.965145 & 2.451249 \\ \text { Si } & 2.853095 & 0.213428 & 2.122654 \\ \text { Si } & 1.345639 & -1.515629 & 1.583003 \\ \text { c } & -3.511227 & 1.686212 & -2.130853 \\ \text { h } & 4.363252 & -0.728253 & 0.124215 \\ \text { h } & 3.400074 & 3.149668 & 1.140906 \\ \text { h } & 4.168642 & 2.247093 & -1.782002 \\ \text { h } & 2.377421 & -3.596271 & -0.425804 \\ \text { h } & 1.664460 & -2.676787 & 2.454858 \\ \text { h } & 1.936541 & 1.628971 & -2.410715 \\ \text { h } & 4.744185 & -1.386510 & -2.130145 \\ \text { h } & 3.258135 & 0.353006 & 3.552152 \\ \text { h } & 0.488469 & 3.268924 & 1.957743 \\ \text { h } & 1.061987 & 4.125063 & 0.552963 \\ \text { h } & 0.968490 & -2.838664 & -2.842895 \\ \text { h } & -0.217469 & -3.171255 & -1.593506 \\ \text { h } & -1.109746 & 3.628895 & -0.031493 \\ \text { h } & -0.223029 & 2.388070 & -0.893462 \\ \text { h } & 0.375854 & -0.398859 & -2.764783 \\ \text { h } & -0.967491 & -1.433154 & -3.214960 \\ \text { h } & -0.076848 & -1.153603 & 1.826157\end{array}$




$\begin{array}{llll}\text { h } & 4.072735 & -0.287360 & 1.387811 \\ \mathrm{~h} & -4.121261 & -0.179579 & -1.587761 \\ \mathrm{~h} & -1.566097 & 1.941303 & 1.664795 \\ \mathrm{~h} & -4.290530 & -1.319058 & 2.649324 \\ \mathrm{~h} & -2.631699 & -1.336412 & 3.272517 \\ \mathrm{~h} & -3.286646 & 0.130070 & 2.534337 \\ \mathrm{~h} & -3.732572 & -3.283940 & 1.184448 \\ \mathrm{~h} & -2.372637 & -3.124609 & 0.054754 \\ \mathrm{~h} & -2.070853 & -3.280707 & 1.794973 \\ \mathrm{~h} & -4.392110 & 1.958315 & -2.724529 \\ \mathrm{~h} & -3.129152 & 2.577665 & -1.634021 \\ \mathrm{~h} & -2.732326 & 1.292805 & -2.780838 \\ \mathrm{~h} & -5.832170 & 1.395228 & -0.809582 \\ \mathrm{~h} & -5.210464 & 0.283020 & 0.438609 \\ \mathrm{~h} & -4.618089 & 1.952130 & 0.363186\end{array}$

Product $\quad \mathrm{RNHTi}\left(\mathrm{N}_{\left.\left(\mathrm{CH}_{3}\right)_{2}\right)=\mathrm{NR} \quad-2994.512899}\right.$

$\begin{array}{llll}\text { c } & 0.880611 & 3.205787 & -0.768579 \\ \mathrm{Si} & 2.136458 & 2.035327 & 0.075374 \\ \mathrm{Si} & 3.115806 & 0.317594 & -1.285881 \\ \mathrm{Si} & 3.141041 & -1.673055 & -0.007091 \\ \mathrm{Si} & 0.931481 & -2.429797 & 0.360806 \\ \mathrm{c} & -0.143305 & -2.925036 & -1.128818 \\ \mathrm{c} & -0.610717 & -1.753390 & -2.017101 \\ \mathrm{n} & -1.233215 & -0.730655 & -1.240372 \\ \mathrm{Ti} & -2.017613 & 0.341478 & -0.170602 \\ \mathrm{c} & -0.406535 & 2.660927 & -1.404471 \\ \mathrm{~h} & -1.369458 & 2.135583 & -0.445088 \\ \mathrm{n} & -3.862828 & -0.081345 & 0.214847 \\ \mathrm{c} & -4.618542 & -1.282300 & -0.090583 \\ \mathrm{C} & -4.684724 & 0.922287 & 0.873588 \\ \mathrm{Si} & 1.759829 & 0.895349 & 2.117677 \\ \mathrm{Si} & 0.102209 & -0.747204 & 1.820396 \\ \mathrm{~h} & 3.429002 & -1.045381 & 1.323817 \\ \mathrm{~h} & 3.269746 & 2.955566 & 0.340603 \\ \mathrm{~h} & 4.522731 & 0.743928 & -1.526859 \\ \mathrm{~h} & 0.986814 & -3.595871 & 1.276011 \\ \mathrm{~h} & -0.198966 & -1.394954 & 3.120427 \\ \mathrm{~h} & 2.447798 & 0.166843 & -2.611214 \\ \mathrm{~h} & 4.208404 & -2.692788 & -0.205606 \\ \mathrm{~h} & 1.776124 & 1.592429 & 3.433582 \\ \mathrm{~h} & 0.629910 & 3.981887 & -0.033337 \\ \mathrm{~h} & 1.474107 & 3.718494 & -1.538480 \\ \mathrm{~h} & 0.404084 & -3.666270 & -1.724693 \\ \mathrm{~h} & -1.021162 & -3.437523 & -0.719407\end{array}$




$\begin{array}{llll}\text { h } & -0.862466 & 3.468570 & -1.997611 \\ \text { h } & -0.166560 & 1.864782 & -2.111626 \\ \text { h } & 0.248602 & -1.355936 & -2.578998 \\ \text { h } & -1.311418 & -2.142259 & -2.771346 \\ \text { h } & -1.273396 & -0.121448 & 1.563131 \\ \text { h } & 2.939235 & -0.049366 & 2.126029 \\ \text { h } & -1.760208 & 2.902058 & 0.103023 \\ \text { h } & -5.526011 & 1.249374 & 0.243222 \\ \text { h } & -5.108888 & 0.549333 & 1.818886 \\ \text { h } & -4.099370 & 1.818418 & 1.116074 \\ \text { h } & -5.473255 & -1.071174 & -0.752881 \\ \text { h } & -3.979498 & -2.012308 & -0.588244 \\ \text { h } & -5.025630 & -1.748668 & 0.820885\end{array}$

Transition $\quad \mathrm{RNHTi}\left(\mathrm{N}_{\left(\mathrm{CH}_{3}\right.} \underline{2}_{2}\right)=\mathrm{NR}+\mathrm{HN}\left(\mathrm{CH}_{3} \underline{2}_{2}-\right.$

$\begin{array}{llll}\text { c } & -0.164332 & 3.064261 & -0.780233 \\ \mathrm{Si} & 1.513552 & 2.392347 & -0.162723 \\ \mathrm{Si} & 2.732037 & 0.932954 & -1.627847 \\ \mathrm{Si} & 3.530401 & -0.875340 & -0.327977 \\ \mathrm{Si} & 1.718958 & -2.209111 & 0.408175 \\ \mathrm{c} & 0.665531 & -3.152018 & -0.868079 \\ \mathrm{c} & -0.417453 & -2.342581 & -1.611008 \\ \mathrm{n} & -1.279853 & -1.680793 & -0.686303 \\ \mathrm{Ti} & -1.957779 & -0.472171 & 0.307435 \\ \mathrm{c} & -1.206231 & 2.086668 & -1.341490 \\ \mathrm{n} & -1.691577 & 1.174277 & -0.343926 \\ \mathrm{n} & -3.995681 & 0.065264 & 0.063203 \\ \mathrm{c} & -4.707937 & 0.554060 & 1.234016 \\ \mathrm{c} & -4.870713 & -0.435638 & -0.983729 \\ \mathrm{Si} & 1.809308 & 1.342540 & 1.938152 \\ \mathrm{Si} & 0.679291 & -0.720016 & 1.963306 \\ \mathrm{~h} & 3.824301 & -0.100683 & 0.922469 \\ \mathrm{~h} & 2.357013 & 3.612656 & -0.106400 \\ \mathrm{~h} & 3.898326 & 1.724524 & -2.108550 \\ \mathrm{~h} & 2.254441 & -3.236623 & 1.337443 \\ \mathrm{~h} & 0.776299 & -1.341911 & 3.305821 \\ \mathrm{~h} & 1.945148 & 0.501581 & -2.820436 \\ \mathrm{~h} & 4.805913 & -1.560104 & -0.679729 \\ \mathrm{~h} & 1.818643 & 2.097538 & 3.221025 \\ \mathrm{~h} & -0.612190 & 3.631677 & 0.043820 \\ \mathrm{~h} & 0.098240 & 3.800700 & -1.551366 \\ \mathrm{~h} & 1.346441 & -3.630637 & -1.583712 \\ \mathrm{~h} & 0.178835 & -3.961114 & -0.312461 \\ \mathrm{~h} & -2.036103 & 2.681960 & -1.756012 \\ \mathrm{~h} & -0.771062 & 1.544438 & -2.194373\end{array}$




$\begin{array}{llll}\text { h } & -0.926328 & -0.248098 & 1.908207 \\ \text { h } & 3.322149 & 0.201848 & 2.144786 \\ \text { h } & -4.451089 & 1.216084 & 0.907229 \\ \text { h } & -5.856277 & -0.813793 & 0.826060 \\ \text { h } & -4.350660 & -1.665495 & 0.377727 \\ \text { h } & -4.493020 & -0.894173 & 1.964642 \\ \text { h } & -5.717583 & 0.727552 & -1.103521 \\ \text { h } & -4.185267 & 1.602610 & -1.398589 \\ \text { h } & -4.265182 & -0.137476 & -1.681167\end{array}$

\begin{tabular}{lll} 
Product & $\mathrm{RN}=\mathrm{Ti}=\mathrm{NR}$ & -2859.224619 \\
\hline
\end{tabular}

$\begin{array}{llll}\text { c } & 0.743164 & 3.118723 & -0.622834 \\ \mathrm{c} & -0.643636 & 2.764149 & -1.184818 \\ \mathrm{n} & -1.498680 & 2.151459 & -0.215553 \\ \mathrm{Ti} & -2.551108 & 0.950550 & 0.404476 \\ \mathrm{n} & -2.623273 & -0.428685 & -0.614229 \\ \mathrm{c} & -2.168363 & -1.318958 & -1.631462 \\ \mathrm{c} & -1.611536 & -2.615293 & -1.004242 \\ \mathrm{Si} & -0.238571 & -2.396008 & 0.297040 \\ \mathrm{Si} & -0.404575 & -0.653255 & 1.924360 \\ \mathrm{Si} & 1.614930 & 0.550014 & 1.966559 \\ \mathrm{Si} & 1.895713 & 1.697697 & -0.080864 \\ \mathrm{Si} & 2.219993 & -0.102361 & -1.633941 \\ \mathrm{Si} & 1.998968 & -2.122105 & -0.424062 \\ \mathrm{~h} & 2.638742 & -1.656511 & 0.849592 \\ \mathrm{~h} & 3.237073 & 2.332809 & -0.001146 \\ \mathrm{~h} & 3.627282 & 0.017798 & -2.107737 \\ \mathrm{~h} & -0.294640 & -3.596683 & 1.170348 \\ \mathrm{~h} & -0.626926 & -1.305931 & 3.242225 \\ \mathrm{~h} & 1.323133 & -0.019940 & -2.824506 \\ \mathrm{~h} & 2.759248 & -3.338426 & -0.828703 \\ \mathrm{~h} & 2.001601 & 1.146467 & 3.273983 \\ \mathrm{~h} & 0.621305 & 3.789153 & 0.235890 \\ \mathrm{~h} & 1.312137 & 3.682036 & -1.374382 \\ \mathrm{~h} & -1.245071 & -3.297102 & -1.782517 \\ \mathrm{~h} & -2.429184 & -3.133099 & -0.490927 \\ \mathrm{~h} & -1.105181 & 3.697524 & -1.547777 \\ \mathrm{~h} & -0.519598 & 2.126640 & -2.074714 \\ \mathrm{~h} & -1.396837 & -0.856520 & -2.267382 \\ \mathrm{~h} & -2.994678 & -1.594778 & -2.305999 \\ \mathrm{~h} & -1.586555 & 0.335490 & 1.969654 \\ \mathrm{~h} & 2.528021 & -0.627904 & 1.746942\end{array}$

Side Imine formation (Figure 10) 


\begin{tabular}{|c|c|c|c|}
\hline \multicolumn{2}{|c|}{ Transition } & \multicolumn{2}{|c|}{$\left.\mathrm{i}\left(\mathrm{N}\left(\mathrm{CH}_{3}\right)_{2}\right) 2--\mathrm{N}\left(\mathrm{CH}_{3}\right)_{2}\right)---\mathrm{H}--\mathrm{NRRNH}$} \\
\hline C & 0.204365 & -1.393513 & -0.242844 \\
\hline Si & 1.850440 & -2.154481 & 0.349175 \\
\hline Si & 2.885201 & -0.977386 & 2.155951 \\
\hline Si & 5.065605 & -0.434698 & 1.442828 \\
\hline $\mathrm{Si}$ & 4.972062 & 1.119150 & -0.331032 \\
\hline c & 4.176539 & 2.825647 & -0.014372 \\
\hline C & 2.678873 & 2.845229 & 0.297204 \\
\hline$n$ & 2.192646 & 4.229258 & 0.377148 \\
\hline c & -0.918945 & -1.376552 & 0.814799 \\
\hline$n$ & -2.122743 & -0.740384 & 0.351123 \\
\hline $\mathrm{Ti}$ & -3.610201 & 0.185184 & 0.074254 \\
\hline $\mathrm{n}$ & -3.371519 & 1.644029 & -1.161144 \\
\hline C & -4.372537 & 2.684253 & -1.350653 \\
\hline$n$ & -4.704216 & 0.491374 & 1.621175 \\
\hline C & -6.106107 & 0.829241 & 1.436397 \\
\hline $\mathrm{n}$ & -4.184486 & -1.518532 & -1.007111 \\
\hline C & -4.400093 & -1.481452 & -2.444841 \\
\hline C & -4.341022 & 0.380152 & 3.022433 \\
\hline Si & 3.560297 & -2.288627 & -1.260439 \\
\hline Si & 4.002592 & -0.141095 & -2.105424 \\
\hline c & -4.946019 & -2.561930 & -0.335209 \\
\hline C & -2.186436 & 1.915136 & -1.961338 \\
\hline h & 5.316198 & -1.688803 & 0.660823 \\
\hline h & 1.632030 & -3.525666 & 0.888947 \\
\hline h & 2.949568 & -1.945193 & 3.279595 \\
\hline h & 6.362672 & 1.348203 & -0.807298 \\
\hline h & 5.067999 & -0.253648 & -3.132846 \\
\hline h & 2.083959 & 0.195871 & 2.616967 \\
\hline h & 6.171761 & -0.347821 & 2.430138 \\
\hline h & 3.627070 & -3.418025 & -2.219719 \\
\hline h & 0.390988 & -0.368140 & -0.580152 \\
\hline h & -0.137961 & -1.945125 & -1.126095 \\
\hline h & 4.731942 & 3.303947 & 0.803147 \\
\hline h & 4.354468 & 3.445371 & -0.900103 \\
\hline h & -1.129130 & -2.410292 & 1.128326 \\
\hline h & -0.561859 & -0.856852 & 1.714292 \\
\hline h & 2.127759 & 2.356905 & -0.513410 \\
\hline h & 2.480204 & 2.261260 & 1.209541 \\
\hline h & 2.816116 & 0.521345 & -2.724911 \\
\hline & 4.732538 & -2.424517 & -0.335832 \\
\hline b & 1.187035 & 4.236215 & 0.520750 \\
\hline & 2.602187 & 4.691198 & 1.184472 \\
\hline & -2.894195 & -1.455423 & -0.487952 \\
\hline
\end{tabular}




$\begin{array}{llll}\mathrm{h} & -2.427728 & 1.957310 & -3.035210 \\ \mathrm{~h} & -1.732214 & 2.883622 & -1.698267 \\ \mathrm{~h} & -1.439404 & 1.136856 & -1.811582 \\ \mathrm{~h} & -4.703202 & 2.741001 & -2.399149 \\ \mathrm{~h} & -5.258259 & 2.505893 & -0.735425 \\ \mathrm{~h} & -3.983740 & 3.677635 & -1.078317 \\ \mathrm{~h} & -6.031332 & -2.399348 & -0.412693 \\ \mathrm{~h} & -4.731801 & -3.554873 & -0.758718 \\ \mathrm{~h} & -4.689753 & -2.584749 & 0.726092 \\ \mathrm{~h} & -5.437325 & -1.214721 & -2.699569 \\ \mathrm{~h} & -3.746733 & -0.738396 & -2.903877 \\ \mathrm{~h} & -4.189324 & -2.456776 & -2.908891 \\ \mathrm{~h} & -4.509128 & 1.323788 & 3.564798 \\ \mathrm{~h} & -4.931017 & -0.396992 & 3.532906 \\ \mathrm{~h} & -3.286750 & 0.117385 & 3.122377 \\ \mathrm{~h} & -6.354918 & 1.824654 & 1.835793 \\ \mathrm{~h} & -6.375047 & 0.824555 & 0.373145 \\ \mathrm{~h} & -6.765501 & 0.100711 & 1.931720\end{array}$

Adduct2 $\quad \mathrm{Ti}\left(\mathrm{N}_{(}\left(\mathrm{CH}_{3}\right)_{2}\right) 2=\mathrm{NRRNH}_{2}{ }^{*}---\mathrm{HN}\left(\mathrm{CH}_{3}\right)_{2} \quad-3264.941277$

$\begin{array}{llll}\text { c } & 0.181829 & -1.283464 & -0.262723 \\ \mathrm{Si} & 1.826344 & -2.150906 & 0.159159 \\ \mathrm{Si} & 3.000253 & -1.234347 & 2.031541 \\ \mathrm{Si} & 5.165292 & -0.693632 & 1.270497 \\ \mathrm{Si} & 5.051367 & 1.061753 & -0.305346 \\ \mathrm{c} & 4.333938 & 2.741507 & 0.249527 \\ \mathrm{c} & 2.842710 & 2.770729 & 0.591002 \\ \mathrm{n} & 2.415072 & 4.143067 & 0.893992 \\ \mathrm{c} & -0.881114 & -1.303930 & 0.859479 \\ \mathrm{n} & -2.106282 & -0.666320 & 0.488548 \\ \mathrm{Ti} & -3.546697 & 0.174378 & 0.121689 \\ \mathrm{n} & -3.211146 & 1.793382 & -0.929079 \\ \mathrm{c} & -4.202993 & 2.841857 & -1.103817 \\ \mathrm{n} & -4.786512 & 0.106371 & 1.626715 \\ \mathrm{c} & -6.071582 & 0.783853 & 1.610809 \\ \mathrm{n} & -4.385879 & -1.213486 & -1.394568 \\ \mathrm{c} & -5.356027 & -0.638823 & -2.346794 \\ \mathrm{c} & -4.566653 & -0.549004 & 2.905417 \\ \mathrm{Si} & 3.450687 & -2.159975 & -1.542510 \\ \mathrm{Si} & 3.944462 & 0.053231 & -2.158144 \\ \mathrm{c} & -4.865328 & -2.466525 & -0.773457 \\ \mathrm{c} & -1.950487 & 2.184962 & -1.537246 \\ \mathrm{~h} & 5.324867 & -1.854559 & 0.335871 \\ \mathrm{~h} & 1.580925 & -3.568467 & 0.546588 \\ \mathrm{~h} & 3.080448 & -2.330959 & 3.030320\end{array}$




$\begin{array}{llll}\mathrm{h} & 6.427522 & 1.293871 & -0.823974 \\ \mathrm{~h} & 4.953052 & 0.026654 & -3.246907 \\ \mathrm{~h} & 2.274979 & -0.094030 & 2.665464 \\ \mathrm{~h} & 6.322730 & -0.764312 & 2.200347 \\ \mathrm{~h} & 3.424178 & -3.168126 & -2.631095 \\ \mathrm{~h} & 0.384800 & -0.244181 & -0.543004 \\ \mathrm{~h} & -0.236421 & -1.760592 & -1.157387 \\ \mathrm{~h} & 4.918903 & 3.085879 & 1.112501 \\ \mathrm{~h} & 4.522141 & 3.467504 & -0.548960 \\ \mathrm{~h} & -1.064581 & -2.351465 & 1.150489 \\ \mathrm{~h} & -0.461473 & -0.818254 & 1.754233 \\ \mathrm{~h} & 2.261258 & 2.436071 & -0.274189 \\ \mathrm{~h} & 2.632070 & 2.060003 & 1.405582 \\ \mathrm{~h} & 2.758813 & 0.825247 & -2.637094 \\ \mathrm{~h} & 4.662112 & -2.445418 & -0.706674 \\ \mathrm{~h} & 1.411168 & 4.166981 & 1.047475 \\ \mathrm{~h} & 2.846276 & 4.453637 & 1.760290 \\ \mathrm{~h} & -3.525526 & -1.419388 & -1.895809 \\ \mathrm{~h} & -2.059101 & 2.373759 & -2.619232 \\ \mathrm{~h} & -1.549561 & 3.113339 & -1.096405 \\ \mathrm{~h} & -1.207091 & 1.400098 & -1.404623 \\ \mathrm{~h} & -4.395747 & 3.057168 & -2.168872 \\ \mathrm{~h} & -5.162368 & 2.570634 & -0.651380 \\ \mathrm{~h} & -3.889393 & 3.791647 & -0.641178 \\ \mathrm{~h} & -5.723466 & -2.242209 & -0.140903 \\ \mathrm{~h} & -5.153231 & -3.209581 & -1.526694 \\ \mathrm{~h} & -4.073036 & -2.865879 & -0.142963 \\ \mathrm{~h} & -6.261723 & -0.351018 & -1.809667 \\ \mathrm{~h} & -4.921873 & 0.251776 & -2.798476 \\ \mathrm{~h} & -5.632948 & -1.353389 & -3.130662 \\ \mathrm{~h} & -4.580018 & 0.168315 & 3.742767 \\ \mathrm{~h} & -5.347739 & -1.298711 & 3.119793 \\ \mathrm{~h} & -3.601426 & -1.053410 & 2.907022 \\ \mathrm{~h} & -6.139062 & 1.578201 & 2.371956 \\ & -6.267330 & 1.254921 & 0.640382 \\ \mathrm{~h} & -6.905988 & 0.088119 & 1.802719\end{array}$

Product $\quad \mathrm{Ti}\left(\mathrm{N}\left(\mathrm{CH}_{3}\right)_{2}\right)_{2}=\mathrm{NRRNH}_{2} \underline{\mathrm{NR}}^{*} \quad-3129.686093$

$\begin{array}{llll}\text { c } & 0.266691 & -1.460988 & 0.525704 \\ \mathrm{c} & 1.399427 & -1.512907 & -0.521817 \\ \mathrm{n} & 2.594214 & -0.881805 & -0.050338 \\ \mathrm{Ti} & 3.978708 & -0.078200 & 0.484691 \\ \mathrm{Si} & -1.384515 & -2.243219 & -0.019994 \\ \mathrm{Si} & -3.131332 & -2.142559 & 1.551547 \\ \mathrm{Si} & -3.527685 & 0.099940 & 2.130562\end{array}$




\begin{tabular}{|c|c|c|c|}
\hline $\mathrm{Si}$ & -4.417718 & 1.171712 & 0.197805 \\
\hline $\mathrm{C}$ & -3.552375 & 2.799182 & -0.298645 \\
\hline c & -2.042479 & 2.726970 & -0.535798 \\
\hline $\mathrm{n}$ & -1.498644 & 4.067962 & -0.787890 \\
\hline $\mathrm{Si}$ & -2.344157 & -1.257625 & -1.976353 \\
\hline $\mathrm{Si}$ & -4.521536 & -0.577029 & -1.385342 \\
\hline $\mathrm{n}$ & 3.734584 & 1.837053 & 0.358696 \\
\hline C & 2.531683 & 2.541573 & -0.052939 \\
\hline $\mathrm{n}$ & 5.556970 & -0.971387 & -0.175055 \\
\hline C & 5.610167 & -2.159025 & -1.012363 \\
\hline C & 4.830986 & 2.771674 & 0.562201 \\
\hline C & 6.890103 & -0.433253 & 0.046565 \\
\hline h & -4.826617 & -1.722874 & -0.469304 \\
\hline h & -1.195922 & -3.674748 & -0.387978 \\
\hline h & -2.411542 & -2.349848 & -2.979346 \\
\hline h & -5.812334 & 1.493166 & 0.607064 \\
\hline h & -4.618910 & 0.139870 & 3.136036 \\
\hline h & -1.499978 & -0.167618 & -2.550158 \\
\hline h & -5.601854 & -0.575756 & -2.404171 \\
\hline h & -3.254933 & -3.148245 & 2.634972 \\
\hline h & 0.090932 & -0.416631 & 0.806922 \\
\hline h & 0.606400 & -1.965193 & 1.438018 \\
\hline h & -4.051665 & 3.181585 & -1.198522 \\
\hline h & -3.746290 & 3.536202 & 0.488238 \\
\hline h & 1.595222 & -2.563489 & -0.786653 \\
\hline h & 1.064060 & -1.023937 & -1.448664 \\
\hline h & -1.547489 & 2.343596 & 0.362502 \\
\hline h & -1.826421 & 2.010577 & -1.343934 \\
\hline h & -2.335675 & 0.797291 & 2.700145 \\
\hline h & -4.287688 & -2.352315 & 0.620932 \\
\hline h & -0.486558 & 4.024120 & -0.862953 \\
\hline h & -1.839605 & 4.416375 & -1.679618 \\
\hline h & 2.201305 & 3.264249 & 0.711728 \\
\hline h & 2.692909 & 3.112501 & -0.982614 \\
\hline h & 1.722645 & 1.833070 & -0.226380 \\
\hline h & 4.590031 & 3.521734 & 1.332642 \\
\hline h & 5.742964 & 2.260324 & 0.888802 \\
\hline h & 5.084730 & 3.323312 & -0.356936 \\
\hline h & 6.063449 & -1.945059 & -1.993899 \\
\hline h & 6.214678 & -2.955905 & -0.548872 \\
\hline h & 4.606071 & -2.545320 & -1.180375 \\
\hline h & 7.375357 & -0.114034 & -0.889355 \\
\hline h & 6.868866 & 0.439238 & 0.709904 \\
\hline h & 7.554419 & -1.175042 & 0.518132 \\
\hline
\end{tabular}


$\underline{\text { Side } \beta \text {-Hydride Elimination }}$

Transition $\quad \mathrm{RNHTi}\left(\mathrm{N}\left(\mathrm{CH}_{3}\right)_{2}\right)-\mathrm{CH}_{2}---\mathrm{H}--\mathrm{N}\left(\mathrm{CH}_{3}\right)_{2} \underline{\mathrm{RNH}} \quad-3129.681956$

$\begin{array}{llll}\text { c } & -0.504279 & -1.972971 & 1.762091 \\ \text { c } & 0.497311 & -2.305328 & 0.621592 \\ \text { n } & 1.816304 & -1.692484 & 0.772759 \\ \text { Ti } & 2.624105 & -0.205626 & -0.160969 \\ \text { n } & 2.688759 & 1.444916 & 1.202862 \\ \text { c } & 2.536459 & 1.252089 & 2.636025 \\ \text { Si } & -2.369098 & -1.876611 & 1.272082 \\ \text { Si } & -3.360770 & 0.168306 & 1.892404 \\ \text { Si } & -2.086816 & 1.927861 & 1.026905 \\ \text { Si } & -2.297503 & 1.862695 & -1.334918 \\ \text { c } & -0.525747 & 1.759395 & -2.067224 \\ \text { c } & 0.150006 & 0.449890 & -1.605180 \\ \text { h } & 1.605047 & 0.382176 & -1.668810 \\ \text { Si } & -2.746645 & -1.968783 & -1.095357 \\ \text { Si } & -3.781016 & 0.059577 & -1.691380 \\ \text { h } & 4.239578 & -0.774758 & -0.859195 \\ \text { c } & 5.041444 & -1.763432 & -1.531672 \\ \text { c } & 4.829816 & 0.296155 & -0.092756 \\ \text { c } & 2.438738 & 2.812144 & 0.774136 \\ \text { h } & -4.624309 & 0.198930 & -0.458908 \\ \text { h } & -3.258144 & -2.954754 & 1.818522 \\ \text { h } & -3.668192 & -3.101782 & -1.305445 \\ \text { h } & -3.037736 & 3.116878 & -1.659462 \\ \text { h } & -2.672595 & 3.224887 & 1.423785 \\ \text { h } & -1.528624 & -2.283706 & -1.892614 \\ \text { h } & -4.728417 & 0.091734 & -2.833408 \\ \text { h } & -3.941506 & 0.389723 & 3.233738 \\ \text { h } & -0.238560 & -0.986120 & 2.151628 \\ \text { h } & -0.379240 & -2.665971 & 2.600985 \\ \text { h } & -0.523804 & 1.832288 & -3.159838 \\ \text { h } & 0.038427 & 2.618689 & -1.689911 \\ \text { h } & 0.586213 & -3.395271 & 0.522594 \\ \text { h } & 0.093026 & -1.968633 & -0.331605 \\ \text { h } & -0.124650 & 0.277668 & -0.555998 \\ \text { h } & -0.292680 & -0.381067 & -2.167544 \\ \text { h } & -0.659814 & 1.908679 & 1.459393\end{array}$




$\begin{array}{llll}\text { h } & -4.479870 & 0.206724 & 0.902831 \\ \mathrm{~h} & 1.959114 & 0.426451 & -2.617704 \\ \mathrm{~h} & 2.406685 & -2.240265 & 1.393686 \\ \mathrm{~h} & 3.141247 & 1.973546 & 3.207154 \\ \mathrm{~h} & 1.492901 & 1.366318 & 2.962161 \\ \mathrm{~h} & 2.856293 & 0.246859 & 2.921765 \\ \mathrm{~h} & 3.045254 & 3.531176 & 1.346886 \\ \mathrm{~h} & 2.689959 & 2.923213 & -0.283159 \\ \mathrm{~h} & 1.385849 & 3.100877 & 0.895558 \\ \mathrm{~h} & 5.660592 & -1.309509 & -2.319018 \\ \mathrm{~h} & 5.722193 & -2.272921 & -0.833104 \\ \mathrm{~h} & 4.399641 & -2.517321 & -1.994683 \\ \mathrm{~h} & 5.328905 & 1.068010 & -0.696179 \\ \mathrm{~h} & 3.869156 & 1.042325 & 0.705358 \\ \mathrm{~h} & 5.507915 & -0.053099 & 0.702158\end{array}$

Adduct2 $\quad \mathrm{RNHTi}\left(\mathrm{N}\left(\mathrm{CH}_{3}\right)_{2}\right)-\mathrm{CH}_{2}-\underline{\mathrm{NHR}}---\mathrm{HN}\left(\mathrm{CH}_{3}\right)_{2}-3129.711004$

$\begin{array}{llll}\text { c } & -0.515928 & -1.639517 & 2.018915 \\ \mathrm{c} & 0.628942 & -2.018106 & 1.040574 \\ \mathrm{n} & 1.863275 & -1.260770 & 1.240864 \\ \mathrm{Ti} & 2.749679 & -0.159713 & -0.101978 \\ \mathrm{n} & 3.194397 & 1.683519 & 1.163145 \\ \mathrm{c} & 2.100216 & 2.107785 & 2.059730 \\ \mathrm{Si} & -2.325489 & -1.814649 & 1.376067 \\ \mathrm{Si} & -3.594100 & 0.127305 & 1.786216 \\ \mathrm{Si} & -2.456434 & 1.979397 & 0.921002 \\ \mathrm{Si} & -2.431533 & 1.768355 & -1.440537 \\ \mathrm{c} & -0.601955 & 1.834615 & -2.008173 \\ \mathrm{c} & 0.164243 & 0.594470 & -1.491379 \\ \mathrm{n} & 1.617584 & 0.677613 & -1.465190 \\ \mathrm{Si} & -2.470886 & -2.072211 & -1.002065 \\ \mathrm{Si} & -3.666538 & -0.212521 & -1.806771 \\ \mathrm{n} & 3.980392 & -1.216512 & -1.061406 \\ \mathrm{c} & 4.238494 & -2.617551 & -1.288451 \\ \mathrm{c} & 4.827747 & -0.398671 & -0.285537 \\ \mathrm{c} & 3.671941 & 2.804441 & 0.323799 \\ \mathrm{~h} & -4.631239 & -0.111091 & -0.661750 \\ \mathrm{~h} & -3.133760 & -2.958903 & 1.916477 \\ \mathrm{~h} & -3.234517 & -3.315227 & -1.215865 \\ \mathrm{~h} & -3.280589 & 2.907373 & -1.900973 \\ \mathrm{~h} & -3.215880 & 3.217749 & 1.189571 \\ \mathrm{~h} & -1.155985 & -2.287144 & -1.665457 \\ \mathrm{~h} & -4.502846 & -0.353199 & -3.025108 \\ \mathrm{~h} & -4.317959 & 0.350491 & 3.057127 \\ \mathrm{~h} & -0.392572 & -0.581009 & 2.267310\end{array}$




$\begin{array}{llll}\text { h } & -0.407125 & -2.180901 & 2.965315 \\ \text { h } & -0.512113 & 1.913834 & -3.096856 \\ \text { h } & -0.156104 & 2.743222 & -1.588052 \\ \text { h } & 0.809607 & -3.100588 & 1.097050 \\ \text { h } & 0.304780 & -1.838647 & 0.013821 \\ \text { h } & -0.161853 & 0.392440 & -0.462627 \\ \text { h } & -0.169927 & -0.267316 & -2.084765 \\ \text { h } & -1.083041 & 2.163663 & 1.477976 \\ \text { h } & -4.615010 & -0.015603 & 0.704303 \\ \text { h } & 1.992804 & 0.773403 & -2.404468 \\ \text { h } & 2.361290 & -1.586834 & 2.066742 \\ \text { h } & 2.389327 & 2.966312 & 2.678489 \\ \text { h } & 1.235495 & 2.388806 & 1.458922 \\ \text { h } & 1.812476 & 1.274181 & 2.698190 \\ \text { h } & 3.925496 & 3.683033 & 0.929484 \\ \text { h } & 4.550902 & 2.481997 & -0.232030 \\ \text { h } & 2.890046 & 3.066826 & -0.387552 \\ \text { h } & 5.047754 & -2.765080 & -2.017378 \\ \text { h } & 4.537020 & -3.135294 & -0.361063 \\ \text { h } & 3.345206 & -3.114484 & -1.679244 \\ \text { h } & 5.516028 & 0.268611 & -0.821093 \\ \text { h } & 3.979125 & 1.375452 & 1.730283 \\ \text { h } & 5.338200 & -0.893568 & 0.563202 \\ & & & \end{array}$

\begin{tabular}{llll} 
Product & \multicolumn{3}{c}{$\mathrm{RNHTi}\left(\mathrm{N}\left(\mathrm{CH}_{3}\right)_{2}\right)-\mathrm{CH}_{2}-\mathrm{NHR}$} \\
c & 0.005489 & 2.589113 & 0.346165 \\
$\mathrm{Si}$ & 1.878090 & 2.283372 & 0.018829 \\
$\mathrm{Si}$ & 2.336839 & 0.925743 & -1.902914 \\
$\mathrm{Si}$ & 3.425380 & -1.005378 & -1.112789 \\
$\mathrm{Si}$ & 1.957141 & -2.267598 & 0.235151 \\
$\mathrm{c}$ & 0.239899 & -2.755331 & -0.455013 \\
$\mathrm{c}$ & -0.482174 & -1.577719 & -1.149492 \\
$\mathrm{n}$ & -1.937578 & -1.612177 & -1.077068 \\
$\mathrm{Ti}$ & -2.883413 & -0.134405 & -0.298632 \\
$\mathrm{n}$ & -4.659767 & -0.422111 & 0.179245 \\
$\mathrm{c}$ & -3.924684 & -0.650376 & 1.398043 \\
$\mathrm{c}$ & -1.001922 & 2.269896 & -0.791557 \\
$\mathrm{n}$ & -2.253141 & 1.685636 & -0.312933 \\
$\mathrm{Si}$ & 2.864683 & 1.078656 & 1.780969 \\
$\mathrm{Si}$ & 1.639592 & -0.884708 & 2.138607 \\
$\mathrm{c}$ & -5.858490 & 0.395170 & 0.195092 \\
$\mathrm{~h}$ & 4.219625 & -0.331812 & -0.033639 \\
$\mathrm{~h}$ & 2.718263 & 3.504751 & -0.195934 \\
$\mathrm{~h}$ & 3.250859 & 1.725500 & -2.745313 \\
$\mathrm{~h}$ & 2.724556 & -3.429095 & 0.768346
\end{tabular}




$\begin{array}{llll}\text { h } & 2.237700 & -1.642331 & 3.262825 \\ \mathrm{~h} & 1.134443 & 0.644315 & -2.737250 \\ \mathrm{~h} & 4.417014 & -1.695024 & -1.973547 \\ \mathrm{~h} & 3.392215 & 1.750902 & 2.986105 \\ \mathrm{~h} & -0.252277 & 1.939901 & 1.188506 \\ \mathrm{~h} & -0.134133 & 3.612582 & 0.710781 \\ \mathrm{~h} & 0.305465 & -3.615258 & -1.129604 \\ \mathrm{~h} & -0.362723 & -3.073235 & 0.402583 \\ \mathrm{~h} & -1.192605 & 3.177277 & -1.379742 \\ \mathrm{~h} & -0.566633 & 1.559843 & -1.497725 \\ \mathrm{~h} & -0.167137 & -0.633917 & -0.677620 \\ \mathrm{~h} & -0.127674 & -1.506788 & -2.186994 \\ \mathrm{~h} & 0.189815 & -0.662378 & 2.424417 \\ \mathrm{~h} & 4.021022 & 0.489493 & 1.044079 \\ \mathrm{~h} & -2.319616 & -2.488033 & -1.418502 \\ \mathrm{~h} & -2.805579 & 2.370053 & 0.200428 \\ \mathrm{~h} & -6.725514 & -0.188314 & 0.527949 \\ \mathrm{~h} & -5.765818 & 1.257021 & 0.875408 \\ \mathrm{~h} & -6.081272 & 0.771977 & -0.807650 \\ \mathrm{~h} & -3.908332 & -1.673441 & 1.777675 \\ \mathrm{~h} & -4.106045 & 0.087754 & 2.190251\end{array}$

\section{TDMAT deposition on -OH SAM on diagonal dimer substrate (Figure 12)}

OH- Surface

$\begin{array}{llll}\text { c } & -2.330662 & 1.946846 & 0.063928 \\ \text { c } & -0.962733 & 2.543834 & -0.212758 \\ \text { o } & -1.167456 & 3.896232 & -0.619165 \\ \mathrm{Si} & -2.3894 & 0.125087 & 0.639972 \\ \mathrm{Si} & -2.014791 & -1.457275 & -1.089003 \\ \mathrm{Si} & -0.031321 & -0.864995 & -2.206452 \\ \mathrm{Si} & 1.731855 & -1.356843 & -0.625324 \\ \mathrm{c} & 3.087739 & -0.040826 & -0.336399 \\ \mathrm{c} & 2.707274 & 1.286353 & 0.291478 \\ \mathrm{o} & 3.897013 & 2.068049 & 0.394064 \\ \mathrm{Si} & -0.640348 & -0.410551 & 2.244824 \\ \mathrm{Si} & 0.550756 & -2.189566 & 1.240386 \\ \mathrm{~h} & -0.536652 & -2.864872 & 0.563907 \\ \text { h } & -3.650409 & -0.270986 & 1.333413 \\ \text { h } & -1.399121 & -0.94563 & 3.415825 \\ \text { h } & 2.458363 & -2.494158 & -1.270361 \\ \text { h } & 0.197991 & -1.853975 & -3.30314 \\ \text { h } & 0.184751 & 0.749044 & 2.689317\end{array}$




$\begin{array}{llll}\text { h } & 1.242718 & -3.184512 & 2.111789 \\ \mathrm{~h} & -3.141158 & -1.891574 & -1.966898 \\ \mathrm{~h} & -2.943758 & 2.055368 & -0.837346 \\ \mathrm{~h} & -2.817489 & 2.563328 & 0.828548 \\ \mathrm{~h} & 3.860772 & -0.508775 & 0.283893 \\ \mathrm{~h} & 3.561145 & 0.154723 & -1.305184 \\ \mathrm{~h} & -0.339779 & 2.504791 & 0.691027 \\ \mathrm{~h} & -0.446047 & 1.98582 & -1.003886 \\ \mathrm{~h} & -0.314347 & 4.281137 & -0.849172 \\ \mathrm{~h} & 1.963198 & 1.803734 & -0.330651 \\ \mathrm{~h} & 2.263658 & 1.13176 & 1.284087 \\ \mathrm{~h} & 3.688684 & 2.888897 & 0.854068 \\ \mathrm{~h} & -0.011542 & 0.507533 & -2.788231 \\ \mathrm{~h} & -1.561668 & -2.585161 & -0.299332\end{array}$

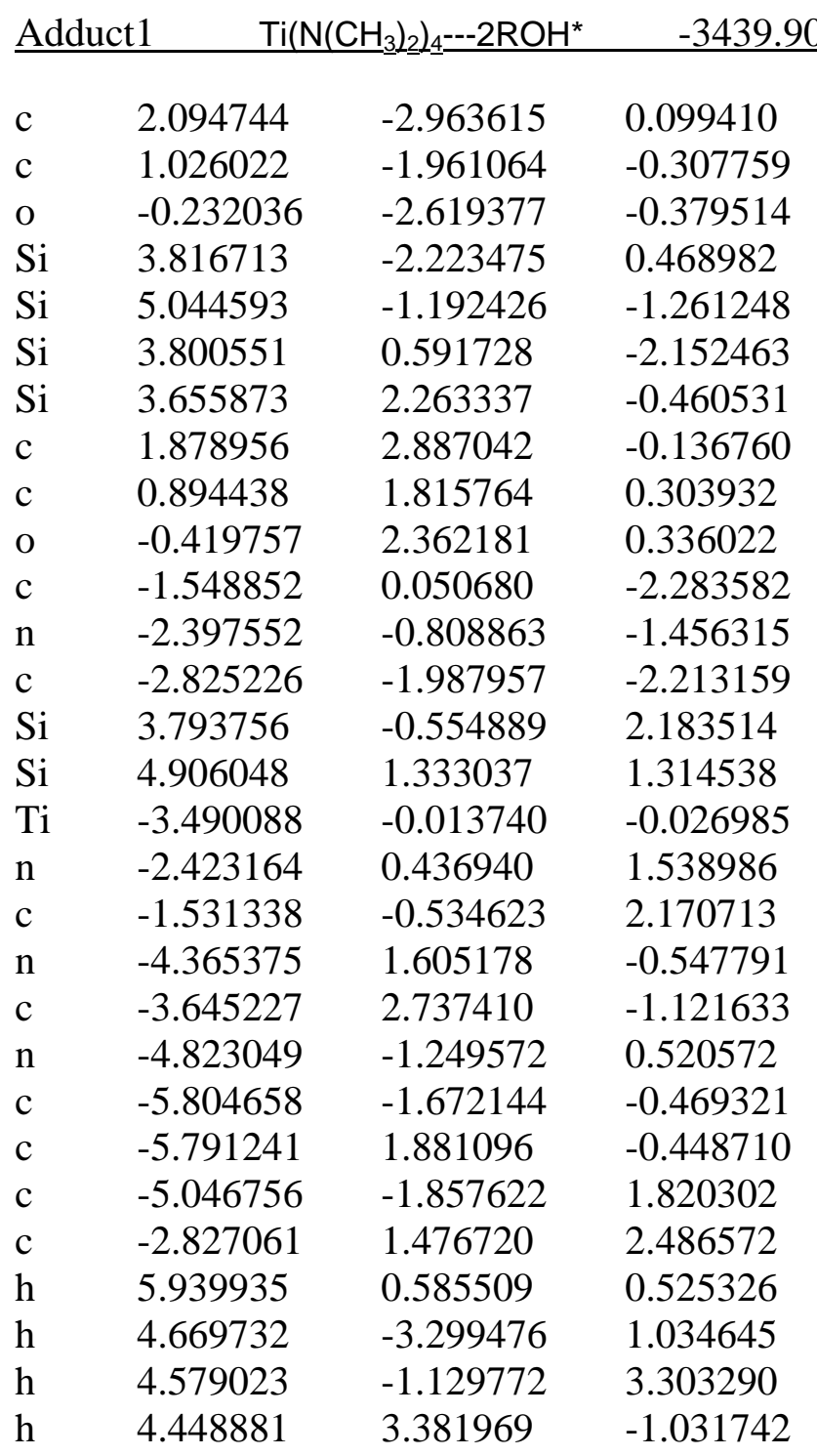




\begin{tabular}{llll} 
h & 4.576440 & 1.212156 & -3.250837 \\
$\mathrm{~h}$ & 2.418888 & -0.275281 & 2.693852 \\
$\mathrm{~h}$ & 5.664715 & 2.230562 & 2.221036 \\
$\mathrm{~h}$ & 5.861624 & -1.983786 & -2.214876 \\
$\mathrm{~h}$ & 2.184905 & -3.740149 & -0.667727 \\
$\mathrm{~h}$ & 1.763885 & -3.473325 & 1.011809 \\
$\mathrm{~h}$ & 1.897333 & 3.699177 & 0.597627 \\
$\mathrm{~h}$ & 1.520110 & 3.328609 & -1.073771 \\
$\mathrm{~h}$ & 0.983138 & -1.147373 & 0.428158 \\
$\mathrm{~h}$ & 1.280099 & -1.512540 & -1.278094 \\
$\mathrm{~h}$ & -0.917013 & -1.968043 & -0.626365 \\
$\mathrm{~h}$ & 0.931892 & 0.970770 & -0.395629 \\
$\mathrm{~h}$ & 1.171550 & 1.433601 & 1.295329 \\
$\mathrm{~h}$ & -1.035571 & 1.688070 & 0.683748 \\
$\mathrm{~h}$ & 2.470421 & 0.192503 & -2.700092 \\
$\mathrm{~h}$ & 6.009726 & -0.399941 & -0.423587 \\
$\mathrm{~h}$ & -5.978079 & 2.771558 & 0.170068 \\
$\mathrm{~h}$ & -6.239610 & 2.074718 & -1.435291 \\
$\mathrm{~h}$ & -6.317839 & 1.043523 & 0.008168 \\
$\mathrm{~h}$ & -3.859169 & 3.658177 & -0.559822 \\
$\mathrm{~h}$ & -2.564123 & 2.593345 & -1.086933 \\
$\mathrm{~h}$ & -3.934469 & 2.916519 & -2.167515 \\
$\mathrm{~h}$ & -6.039715 & -1.599421 & 2.217406 \\
$\mathrm{~h}$ & -4.990410 & -2.954620 & 1.766629 \\
$\mathrm{~h}$ & -4.299245 & -1.517828 & 2.537483 \\
$\mathrm{~h}$ & -6.827883 & -1.422378 & -0.153064 \\
$\mathrm{~h}$ & -5.631981 & -1.180691 & -1.431866 \\
$\mathrm{~h}$ & -5.767251 & -2.757080 & -0.642425 \\
$\mathrm{~h}$ & -1.959129 & -2.559750 & -2.572160 \\
$\mathrm{~h}$ & -3.414476 & -2.654932 & -1.583409 \\
$\mathrm{~h}$ & -3.427177 & -1.719691 & -3.095330 \\
$\mathrm{~h}$ & -0.674467 & -0.500740 & -2.658076 \\
$\mathrm{~h}$ & -2.082996 & 0.441933 & -3.162061 \\
$\mathrm{~h}$ & -1.175207 & 0.900979 & -1.709361 \\
$\mathrm{~h}$ & -1.956503 & 2.019581 & 2.881243 \\
$\mathrm{~h}$ & -3.481685 & 2.197368 & 1.997684 \\
$\mathrm{~h}$ & -3.365495 & 1.052507 & 3.347313 \\
$\mathrm{~h}$ & -1.246003 & -1.325332 & 1.475656 \\
$\mathrm{~h}$ & -0.610384 & -0.056905 & 2.536013 \\
\hline & -2.004441 & -1.012525 & 3.041882 \\
\hline
\end{tabular}

Transition $\quad \mathrm{Ti}\left(\mathrm{N}\left(\mathrm{CH}_{3}\right)_{2}\right)_{4}{ }_{4}---\mathrm{H}_{---\mathrm{ORROH}^{*}} \quad-3439.88904$

$\begin{array}{llll}\text { C } & 1.051897 & 2.537212 & 0.029881 \\ \text { C } & 0.287744 & 1.210984 & -0.004898 \\ \text { o } & -1.101308 & 1.380466 & 0.210188\end{array}$




\begin{tabular}{|c|c|c|c|}
\hline SI & 2.937800 & 2.388752 & -0.283970 \\
\hline $\mathrm{Si}$ & 4.309080 & 1.639599 & 1.484925 \\
\hline $\mathrm{Si}$ & 3.596119 & -0.483963 & 2.175656 \\
\hline $\mathrm{Si}$ & 4.059498 & -1.997594 & 0.400392 \\
\hline $\mathrm{C}$ & 2.491381 & -2.989807 & -0.050310 \\
\hline C & 1.445540 & -2.146725 & -0.782408 \\
\hline o & 0.139475 & -2.557617 & -0.401008 \\
\hline C & -1.457678 & -0.961215 & 2.480133 \\
\hline $\mathrm{n}$ & -2.270435 & 0.124589 & 1.925292 \\
\hline C & -3.062205 & 0.793286 & 2.954971 \\
\hline SI & 3.523667 & 0.918615 & -2.082756 \\
\hline SI & 5.086743 & -0.622018 & -1.219987 \\
\hline $\mathrm{Ti}$ & -2.996517 & 0.084881 & -0.064703 \\
\hline $\mathrm{n}$ & -2.152012 & -0.868426 & -1.573186 \\
\hline C & -1.557871 & -0.113173 & -2.676061 \\
\hline $\mathrm{n}$ & -4.440134 & -1.114939 & 0.303006 \\
\hline C & -4.746966 & -1.983154 & 1.423776 \\
\hline $\mathrm{n}$ & -3.972356 & 1.678543 & -0.474403 \\
\hline C & -5.330003 & 1.980530 & -0.040959 \\
\hline C & -5.438169 & -1.260934 & -0.753966 \\
\hline C & -3.430858 & 2.775926 & -1.267547 \\
\hline C & -2.577188 & -2.184000 & -2.058201 \\
\hline h & 5.800456 & 0.334673 & -0.309604 \\
\hline h & 3.472166 & 3.707506 & -0.720789 \\
\hline h & 4.174115 & 1.779507 & -3.098691 \\
\hline h & 5.107837 & -2.882277 & 0.970040 \\
\hline h & 4.444112 & -0.940312 & 3.301964 \\
\hline h & 2.340924 & 0.286801 & -2.736464 \\
\hline h & 6.134489 & -1.188966 & -2.105378 \\
\hline h & 4.789052 & 2.559163 & 2.543838 \\
\hline h & 0.886389 & 3.033618 & 0.992328 \\
\hline h & 0.639592 & 3.206246 & -0.733602 \\
\hline h & 2.731392 & -3.899762 & -0.607194 \\
\hline h & 2.054118 & -3.315380 & 0.899475 \\
\hline h & 0.439612 & 0.733972 & -0.976988 \\
\hline h & 0.694870 & 0.526848 & 0.752224 \\
\hline h & -1.459058 & 0.971689 & 1.232471 \\
\hline h & 1.577126 & -1.086303 & -0.540242 \\
\hline h & 1.586191 & -2.237861 & -1.868647 \\
\hline h & -0.513298 & -1.917576 & -0.743258 \\
\hline h & 2.170482 & -0.536197 & 2.616006 \\
\hline h & 5.514003 & 1.224661 & 0.691505 \\
\hline h & -5.476496 & -2.292758 & -1.128719 \\
\hline h & -6.443158 & -1.006929 & -0.387412 \\
\hline h & -5.217828 & -0.605591 & -1.598474 \\
\hline h & -4.779471 & -3.038943 & 1.114913 \\
\hline
\end{tabular}




$\begin{array}{llll}\text { h } & -4.001221 & -1.887853 & 2.208525 \\ \mathrm{~h} & -5.731042 & -1.745208 & 1.855783 \\ \mathrm{~h} & -3.997707 & 2.913731 & -2.200276 \\ \mathrm{~h} & -3.480353 & 3.721126 & -0.707830 \\ \mathrm{~h} & -2.385400 & 2.603938 & -1.511801 \\ \mathrm{~h} & -6.004990 & 2.133595 & -0.896867 \\ \mathrm{~h} & -5.730618 & 1.176769 & 0.573809 \\ \mathrm{~h} & -5.351694 & 2.905901 & 0.553598 \\ \mathrm{~h} & -2.422488 & 1.160457 & 3.770444 \\ \mathrm{~h} & -3.585371 & 1.650861 & 2.528400 \\ \mathrm{~h} & -3.811214 & 0.126761 & 3.403663 \\ \mathrm{~h} & -0.756145 & -0.583593 & 3.237938 \\ \mathrm{~h} & -2.070643 & -1.730121 & 2.969228 \\ \mathrm{~h} & -0.875972 & -1.454185 & 1.698058 \\ \mathrm{~h} & -1.730978 & -2.734160 & -2.491511 \\ \mathrm{~h} & -2.974641 & -2.786397 & -1.242975 \\ \mathrm{~h} & -3.345919 & -2.105760 & -2.841852 \\ \mathrm{~h} & -1.262339 & 0.885410 & -2.356373 \\ \mathrm{~h} & -0.662022 & -0.614110 & -3.072649 \\ \mathrm{~h} & -2.258684 & -0.002579 & -3.517677\end{array}$

Adduct2 $\quad \mathrm{Ti}\left(\mathrm{N}_{(}\left(\mathrm{CH}_{3}\right)_{2} 2_{3}---\mathrm{ORROH}^{*}{ }_{--\mathrm{HN}}(\mathrm{CH} 3)_{2} 2_{4}\right.$ $-3439.937377$

$\begin{array}{llll}\text { c } & -0.782971 & -2.239930 & -0.965680 \\ \mathrm{Si} & -2.693564 & -2.198735 & -1.119044 \\ \mathrm{Si} & -3.549067 & -0.285516 & -2.276206 \\ \mathrm{Si} & -5.189136 & 0.672916 & -0.877377 \\ \mathrm{Si} & -4.184009 & 1.532829 & 1.078128 \\ \mathrm{C} & -2.760414 & 2.794366 & 0.934676 \\ \mathrm{C} & -1.669819 & 2.337386 & -0.036813 \\ \mathrm{o} & -0.403791 & 2.788189 & 0.410916 \\ \mathrm{c} & -0.144688 & -0.947151 & -0.453710 \\ \mathrm{o} & 1.257795 & -1.033310 & -0.411466 \\ \mathrm{SI} & -3.991616 & -2.261809 & 0.851508 \\ \mathrm{Si} & -3.444651 & -0.426900 & 2.206324 \\ \mathrm{n} & 3.355837 & -2.104911 & 1.029398 \\ \mathrm{Ti} & 2.734304 & 0.052595 & -0.133580 \\ \mathrm{n} & 4.220188 & -0.349292 & -1.298479 \\ \mathrm{c} & 4.300829 & -1.532648 & -2.134857 \\ \mathrm{C} & 2.521810 & -2.315903 & 2.219653 \\ \mathrm{C} & 4.778048 & -2.324008 & 1.318801 \\ \mathrm{n} & 2.008633 & 1.671847 & -1.039928 \\ \mathrm{C} & 2.532006 & 3.022540 & -0.849731 \\ \mathrm{n} & 3.175709 & 0.937240 & 1.513466 \\ \mathrm{C} & 4.498681 & 1.305299 & 1.980292 \\ \mathrm{c} & 1.542031 & 1.509609 & -2.415074\end{array}$




\begin{tabular}{|c|c|c|c|}
\hline C & 2.129789 & 1.386447 & 2.418369 \\
\hline c & 5.187340 & 0.656846 & -1.699494 \\
\hline h & -5.735232 & -0.613724 & -0.329225 \\
\hline h & -3.121869 & -3.346494 & -1.962828 \\
\hline h & -4.186094 & -0.824525 & -3.502271 \\
\hline h & -5.270681 & 2.036461 & 1.957067 \\
\hline h & -4.247003 & -0.487931 & 3.449261 \\
\hline h & -2.479015 & 0.659199 & -2.707920 \\
\hline h & -6.351086 & 1.377275 & -1.475541 \\
\hline h & -4.299381 & -3.537700 & 1.542298 \\
\hline h & -0.495787 & -3.077217 & -0.319639 \\
\hline h & -0.380001 & -2.465311 & -1.959685 \\
\hline h & -3.122366 & 3.796580 & 0.689077 \\
\hline h & -2.309913 & 2.862638 & 1.930719 \\
\hline h & -0.435650 & -0.117008 & -1.106752 \\
\hline h & -0.527420 & -0.716806 & 0.550405 \\
\hline h & 3.056543 & -2.767830 & 0.320702 \\
\hline h & -1.667455 & 1.242467 & -0.100456 \\
\hline h & -1.892100 & 2.707158 & -1.048127 \\
\hline h & 0.304431 & 2.314394 & -0.071353 \\
\hline h & -2.002674 & -0.363941 & 2.589373 \\
\hline h & -5.288391 & -1.749735 & 0.292416 \\
\hline h & 4.605836 & 2.396806 & 2.083544 \\
\hline h & 4.718040 & 0.869961 & 2.968309 \\
\hline h & 5.268681 & 0.956640 & 1.289261 \\
\hline h & 2.169982 & 2.474692 & 2.573396 \\
\hline h & 1.136884 & 1.167393 & 2.019698 \\
\hline h & 2.212441 & 0.911125 & 3.408007 \\
\hline h & 4.172244 & -1.288297 & -3.200962 \\
\hline h & 5.274965 & -2.039937 & -2.040009 \\
\hline h & 3.520331 & -2.251289 & -1.875425 \\
\hline h & 5.060195 & 0.960559 & -2.749999 \\
\hline h & 5.100684 & 1.556186 & -1.085834 \\
\hline h & 6.218789 & 0.285341 & -1.592636 \\
\hline h & 4.979265 & -3.326230 & 1.722774 \\
\hline h & 5.362174 & -2.177484 & 0.411585 \\
\hline h & 5.108752 & -1.589973 & 2.055370 \\
\hline h & 2.672070 & -3.305716 & 2.673379 \\
\hline h & 2.763600 & -1.556754 & 2.964733 \\
\hline h & 1.473199 & -2.207317 & 1.945623 \\
\hline h & 1.766172 & 3.783441 & -1.058092 \\
\hline h & 2.865645 & 3.159618 & 0.178280 \\
\hline h & 3.382428 & 3.236051 & -1.517547 \\
\hline h & 1.171954 & 0.496469 & -2.582568 \\
\hline h & 0.726672 & 2.209863 & -2.654277 \\
\hline h & 2.343597 & 1.694497 & -3.147425 \\
\hline
\end{tabular}


Product $\quad \mathrm{Ti}\left(\mathrm{N}\left(\mathrm{CH}_{3}\right)_{2}\right)_{3}=--\mathrm{OHRROH}^{*} \quad-3304.738129$

\begin{tabular}{|c|c|c|c|}
\hline C & -0.650645 & -2.619997 & -0.072622 \\
\hline C & -0.026040 & -1.374927 & 0.565870 \\
\hline 0 & 1.307485 & -1.169889 & 0.165220 \\
\hline $\mathrm{Ti}$ & 2.857584 & -0.234371 & 0.081635 \\
\hline $\mathrm{n}$ & 3.338410 & 0.719821 & 1.661235 \\
\hline C & 2.478805 & 1.789626 & 2.154805 \\
\hline $\mathrm{Si}$ & -2.547751 & -2.472637 & -0.282864 \\
\hline $\mathrm{Si}$ & -3.874764 & -1.657080 & 1.490198 \\
\hline $\mathrm{Si}$ & -3.079371 & 0.451385 & 2.164165 \\
\hline $\mathrm{Si}$ & -3.482231 & 1.964881 & 0.364967 \\
\hline $\mathrm{C}$ & -1.944366 & 2.960237 & -0.187897 \\
\hline C & -0.696075 & 2.111653 & -0.383098 \\
\hline 0 & 0.401713 & 2.932848 & -0.748050 \\
\hline $\mathrm{Si}$ & -3.070934 & -0.992178 & -2.083092 \\
\hline $\mathrm{Si}$ & -4.569297 & 0.609023 & -1.234293 \\
\hline $\mathrm{n}$ & 4.272345 & -1.455883 & -0.272197 \\
\hline C & 4.156872 & -2.902956 & -0.171357 \\
\hline $\mathrm{n}$ & 2.747909 & 1.115314 & -1.325992 \\
\hline C & 2.219219 & 0.698946 & -2.625690 \\
\hline C & 5.554502 & -1.035849 & -0.813761 \\
\hline C & 3.663233 & 2.246496 & -1.452262 \\
\hline C & 4.442446 & 0.447986 & 2.567809 \\
\hline h & -5.316515 & -0.315728 & -0.320777 \\
\hline h & -3.116940 & -3.777269 & -0.702818 \\
\hline h & -3.742658 & -1.842643 & -3.096224 \\
\hline h & -4.503999 & 2.882315 & 0.936144 \\
\hline h & -3.917018 & 0.934256 & 3.283412 \\
\hline h & -1.838664 & -0.423761 & -2.706094 \\
\hline h & -5.590381 & 1.205532 & -2.128579 \\
\hline h & -4.393022 & -2.555199 & 2.551263 \\
\hline h & -0.376994 & -3.525289 & 0.477533 \\
\hline h & -0.239824 & -2.732986 & -1.081691 \\
\hline h & -2.167949 & 3.502083 & -1.112931 \\
\hline h & -1.740725 & 3.720684 & 0.574138 \\
\hline h & -0.597707 & -0.485850 & 0.282757 \\
\hline h & -0.076853 & -1.448734 & 1.658583 \\
\hline h & -0.472359 & 1.580393 & 0.551588 \\
\hline h & -0.874201 & 1.351032 & -1.157135 \\
\hline h & 1.172969 & 2.346805 & -0.875922 \\
\hline h & -1.664888 & 0.440342 & 2.637634 \\
\hline h & -5.065196 & -1.208179 & 0.687674 \\
\hline h & 5.079323 & 1.335976 & 2.699810 \\
\hline h & 4.083956 & 0.156652 & 3.566954 \\
\hline h & 5.058935 & -0.362808 & 2.180759 \\
\hline
\end{tabular}




$\begin{array}{llll}\text { h } & 3.062439 & 2.699649 & 2.357208 \\ \text { h } & 1.718030 & 2.062012 & 1.419634 \\ \text { h } & 1.969496 & 1.511647 & 3.089077 \\ \text { h } & 6.387222 & -1.389626 & -0.187737 \\ \text { h } & 5.717384 & -1.428188 & -1.828520 \\ \text { h } & 5.621751 & 0.053556 & -0.863353 \\ \text { h } & 4.913502 & -3.318422 & 0.510752 \\ \text { h } & 3.171764 & -3.180460 & 0.204758 \\ \text { h } & 4.295889 & -3.388393 & -1.149386 \\ \text { h } & 3.165879 & 3.118540 & -1.900871 \\ \text { h } & 4.035823 & 2.539884 & -0.469923 \\ \text { h } & 4.529139 & 2.008027 & -2.089662 \\ \text { h } & 1.560790 & -0.164719 & -2.507167 \\ \text { h } & 1.634048 & 1.501787 & -3.096510 \\ \text { h } & 3.019635 & 0.420024 & -3.326934\end{array}$

\section{Transition $\quad$ RO---H---Ti(N(CH3)2)3OR $\quad-3304.723298$}

$\begin{array}{llll}\text { c } & -0.764657 & 2.862316 & -0.632848 \\ \text { c } & 0.200775 & 1.835995 & -1.243888 \\ \text { o } & 0.880449 & 1.074240 & -0.267185 \\ \mathrm{Ti} & 2.328402 & -0.028178 & -0.114322 \\ \mathrm{n} & 3.082173 & -0.691617 & -1.744883 \\ \mathrm{C} & 3.047001 & -2.077044 & -2.200894 \\ \mathrm{Si} & -2.422377 & 2.338404 & 0.178836 \\ \mathrm{Si} & -4.110759 & 1.350753 & -1.158214 \\ \mathrm{Si} & -3.344873 & -0.700102 & -1.996331 \\ \mathrm{Si} & -3.123501 & -2.168437 & -0.132421 \\ \mathrm{C} & -1.326165 & -2.812587 & 0.046594 \\ \mathrm{c} & -0.389913 & -1.646760 & -0.283902 \\ \mathrm{o} & 0.979304 & -1.856988 & -0.000899 \\ \mathrm{Si} & -2.329536 & 0.890198 & 2.080553 \\ \mathrm{Si} & -3.861887 & -0.858987 & 1.681044 \\ \mathrm{n} & 3.656740 & 1.304738 & 0.291162 \\ \mathrm{c} & 3.408797 & 2.646692 & 0.792274 \\ \mathrm{n} & 2.438896 & -1.088123 & 1.717559 \\ \mathrm{C} & 2.210770 & -0.305760 & 2.926498 \\ \mathrm{C} & 5.077842 & 0.992356 & 0.300533 \\ \mathrm{C} & 3.559585 & -2.011518 & 1.878311 \\ \mathrm{C} & 3.744455 & 0.161352 & -2.723378 \\ \mathrm{~h} & -4.901818 & -0.047127 & 0.981907 \\ \mathrm{~h} & -3.008021 & 3.601466 & 0.718927 \\ \mathrm{~h} & -2.803668 & 1.713569 & 3.220246 \\ \mathrm{~h} & -4.155696 & -3.209693 & -0.398526 \\ \mathrm{~h} & -4.385913 & -1.310436 & -2.845449 \\ \mathrm{~h} & -0.954159 & 0.426355 & 2.411271\end{array}$




$\begin{array}{llll}\text { h } & -4.551042 & -1.515736 & 2.819668 \\ \mathrm{~h} & -4.973530 & 2.151402 & -2.064255 \\ \mathrm{~h} & -1.039810 & 3.574558 & -1.421925 \\ \mathrm{~h} & -0.220197 & 3.450257 & 0.114778 \\ \mathrm{~h} & -1.158980 & -3.157992 & 1.072166 \\ \mathrm{~h} & -1.135982 & -3.663980 & -0.613826 \\ \mathrm{~h} & -0.330843 & 1.161298 & -1.926526 \\ \mathrm{~h} & 0.940770 & 2.376786 & -1.849268 \\ \mathrm{~h} & -0.461947 & -1.426252 & -1.351832 \\ \mathrm{~h} & -0.724470 & -0.744306 & 0.242580 \\ \mathrm{~h} & 1.387137 & -1.717321 & 1.054016 \\ \mathrm{~h} & -2.112743 & -0.581196 & -2.829579 \\ \mathrm{~h} & -5.006817 & 0.824111 & -0.070018 \\ \mathrm{~h} & 4.772001 & -0.179582 & -2.924066 \\ \mathrm{~h} & 3.209260 & 0.152505 & -3.684389 \\ \mathrm{~h} & 3.792133 & 1.190648 & -2.369523 \\ \mathrm{~h} & 4.064759 & -2.480071 & -2.318603 \\ \mathrm{~h} & 2.496533 & -2.698504 & -1.497822 \\ \mathrm{~h} & 2.552658 & -2.158240 & -3.180803 \\ \mathrm{~h} & 5.648537 & 1.714038 & -0.303902 \\ \mathrm{~h} & 5.497593 & 1.020985 & 1.317418 \\ \mathrm{~h} & 5.257757 & -0.001864 & -0.111482 \\ \mathrm{~h} & 3.917292 & 3.402674 & 0.173582 \\ \mathrm{~h} & 2.341133 & 2.861491 & 0.787857 \\ \mathrm{~h} & 3.779295 & 2.774879 & 1.821250 \\ \mathrm{~h} & 3.379682 & -2.711319 & 2.706985 \\ \mathrm{~h} & 3.696501 & -2.607175 & 0.971691 \\ \mathrm{~h} & 4.504008 & -1.490272 & 2.089422 \\ \mathrm{~h} & 1.360780 & 0.366970 & 2.790519 \\ \mathrm{~h} & 1.985132 & -0.956143 & 3.783950 \\ \mathrm{~h} & 3.085272 & 0.304285 & 3.192115 \\ & & & \end{array}$

Adduct2 $\left.\quad\left(\mathrm{CH}_{3}\right)_{2}\right) \mathrm{NH}---\mathrm{RO}---\mathrm{Ti}\left(\mathrm{N}_{2}\left(\mathrm{CH}_{3}\right)_{2}\right)_{2}{ }_{2} \mathrm{OR}$ $-3304.760021$

$\begin{array}{llll}\text { c } & 1.148409 & -2.928237 & -0.509337 \\ \mathrm{C} & 0.104679 & -2.025585 & -1.180557 \\ \mathrm{o} & -0.564908 & -1.205948 & -0.256808 \\ \mathrm{TI} & -2.087315 & -0.176353 & -0.104210 \\ \mathrm{n} & -2.848545 & -1.110418 & 1.416456 \\ \mathrm{C} & -2.064827 & -1.877142 & 2.373441 \\ \mathrm{Si} & 2.679022 & -2.166613 & 0.360515 \\ \mathrm{Si} & 4.327634 & -1.057817 & -0.933429 \\ \mathrm{Si} & 3.386096 & 0.837688 & -1.945962 \\ \mathrm{Si} & 2.872417 & 2.367467 & -0.189791 \\ \mathrm{C} & 0.996292 & 2.765965 & -0.099573 \\ \mathrm{C} & 0.225262 & 1.580828 & -0.704126\end{array}$




\begin{tabular}{|c|c|c|c|}
\hline 0 & -1.100857 & 1.453480 & -0.259366 \\
\hline $\mathrm{Si}$ & 2.289839 & -0.630229 & 2.155556 \\
\hline Si & 3.638630 & 1.258902 & 1.738121 \\
\hline $\mathrm{n}$ & -3.606471 & 1.747359 & 0.354631 \\
\hline C & -3.562226 & 2.251183 & 1.735950 \\
\hline$n$ & -3.039872 & -0.588675 & -1.707558 \\
\hline C & -2.728882 & 0.066365 & -2.967846 \\
\hline C & -4.938429 & 1.886287 & -0.245734 \\
\hline C & -4.035317 & -1.636247 & -1.856918 \\
\hline C & -4.250590 & -1.111984 & 1.789011 \\
\hline h & 4.807551 & 0.543779 & 1.155332 \\
\hline h & 3.373629 & -3.315284 & 1.013836 \\
\hline h & 2.783746 & -1.327279 & 3.372661 \\
\hline h & 3.792083 & 3.510820 & -0.467105 \\
\hline h & 4.405999 & 1.517347 & -2.768959 \\
\hline h & 0.856058 & -0.295577 & 2.370332 \\
\hline h & 4.165663 & 2.061162 & 2.871057 \\
\hline h & 5.338884 & -1.798718 & -1.733741 \\
\hline h & 1.537387 & -3.626514 & -1.262139 \\
\hline h & 0.643476 & -3.549086 & 0.239825 \\
\hline h & 0.714353 & 2.875931 & 0.951841 \\
\hline h & 0.741312 & 3.704881 & -0.599393 \\
\hline h & 0.586463 & -1.402254 & -1.947383 \\
\hline h & -0.625382 & -2.658669 & -1.703813 \\
\hline h & 0.244167 & 1.677105 & -1.799340 \\
\hline h & 0.744899 & 0.643485 & -0.471492 \\
\hline h & -2.940413 & 2.296398 & -0.182207 \\
\hline h & 2.237151 & 0.527306 & -2.845105 \\
\hline h & 5.083576 & -0.366623 & 0.169633 \\
\hline h & -4.995889 & -1.236711 & -2.219431 \\
\hline h & -3.709255 & -2.398800 & -2.580158 \\
\hline h & -4.210565 & -2.136914 & -0.904584 \\
\hline h & -3.622523 & 0.530381 & -3.414631 \\
\hline h & -1.985998 & 0.852830 & -2.820799 \\
\hline h & -2.329432 & -0.642574 & -3.708308 \\
\hline h & -4.641212 & -2.140905 & 1.840972 \\
\hline h & -4.426066 & -0.660857 & 2.779343 \\
\hline h & -4.858009 & -0.573567 & 1.061216 \\
\hline h & -2.434092 & -2.912747 & 2.448062 \\
\hline h & -1.021503 & -1.912161 & 2.068283 \\
\hline h & -2.114870 & -1.444857 & 3.385769 \\
\hline h & -5.299088 & 2.923924 & -0.226341 \\
\hline h & -4.908855 & 1.539143 & -1.278237 \\
\hline h & -5.660400 & 1.273757 & 0.297949 \\
\hline h & -2.535321 & 2.208063 & 2.097060 \\
\hline h & -3.919573 & 3.287457 & 1.812118 \\
\hline
\end{tabular}




h $\quad-4.183840 \quad 1.627821 \quad 2.379199$

Product $\quad \mathrm{ROTi}\left(\mathrm{N}\left(\mathrm{CH}_{3}\right)_{2} 2_{2}{ }_{2} \mathrm{OR} \quad-3169.57036\right.$

$\begin{array}{llll}\text { c } & 0.690266 & -3.129247 & 0.294308 \\ \mathrm{Si} & 2.370800 & -2.218828 & 0.389303 \\ \mathrm{Si} & 2.616536 & -0.548620 & 2.083187 \\ \mathrm{Si} & 3.473262 & 1.375004 & 1.011912 \\ \mathrm{Si} & 1.856913 & 2.249245 & -0.461662 \\ \mathrm{c} & 0.281499 & 2.891467 & 0.418120 \\ \mathrm{c} & -1.007174 & 2.953164 & -0.411191 \\ \mathrm{o} & -1.524298 & 1.670184 & -0.657535 \\ \mathrm{Ti} & -2.071995 & 0.059942 & -0.031936 \\ \mathrm{~h} & -3.463861 & -0.575137 & -1.168117 \\ \mathrm{c} & -4.532877 & -1.474114 & -0.766491 \\ \mathrm{c} & -0.433676 & -2.441496 & -0.476575 \\ \mathrm{o} & -0.716079 & -1.163048 & 0.054210 \\ \mathrm{~h} & -2.786910 & 0.185814 & 1.726854 \\ \mathrm{c} & -3.851105 & 1.145232 & 1.982942 \\ \mathrm{C} & -2.387064 & -0.551076 & 2.913206 \\ \mathrm{Si} & 3.181623 & -1.143151 & -1.549113 \\ \mathrm{Si} & 1.697964 & 0.580351 & -2.163901 \\ \mathrm{c} & -3.575857 & -0.161178 & -2.559540 \\ \mathrm{~h} & 4.351922 & 0.668577 & 0.030613 \\ \mathrm{~h} & 3.361382 & -3.259075 & 0.778088 \\ \mathrm{~h} & 3.643725 & -1.080526 & 3.022403 \\ \mathrm{~h} & 2.471492 & 3.396154 & -1.190707 \\ \mathrm{~h} & 2.235169 & 1.219187 & -3.393863 \\ \mathrm{~h} & 1.366136 & -0.309114 & 2.862093 \\ \mathrm{~h} & 4.358982 & 2.312257 & 1.749808 \\ \mathrm{~h} & 3.824921 & -1.918723 & -2.642378 \\ \mathrm{~h} & 0.884433 & -4.106186 & -0.165567 \\ \mathrm{~h} & 0.359946 & -3.334481 & 1.319015 \\ \mathrm{~h} & 0.082056 & 2.289949 & 1.310924 \\ \mathrm{~h} & 0.528818 & 3.898004 & 0.778621 \\ \mathrm{~h} & -0.155116 & -2.339048 & -1.533716 \\ \mathrm{~h} & -1.339569 & -3.061591 & -0.445020 \\ \mathrm{~h} & -1.759734 & 3.548625 & 0.124285 \\ \mathrm{~h} & -0.832141 & 3.451193 & -1.373536 \\ \mathrm{~h} & 0.321876 & 0.109115 & -2.474044 \\ \mathrm{~h} & 4.268025 & -0.319387 & -0.914681 \\ \mathrm{~h} & -5.522375 & -1.008508 & -0.895337 \\ \mathrm{~h} & -4.529310 & -2.398750 & -1.363047 \\ \mathrm{~h} & -4.425427 & -1.750032 & 0.283620 \\ \mathrm{~h} & -2.781984 & 0.542473 & -2.815326\end{array}$




$\begin{array}{llll}\text { h } & -3.504535 & -1.018790 & -3.244893 \\ \text { h } & -4.759260 & 0.649920 & 2.357491 \\ \text { h } & -3.551764 & 1.896512 & 2.728332 \\ \text { h } & -4.121907 & 1.675405 & 1.065652 \\ \text { h } & -3.224215 & -1.135682 & 3.324734 \\ \text { h } & -1.575846 & -1.238135 & 2.672470 \\ \text { h } & -2.037632 & 0.122303 & 3.710150\end{array}$

$\beta$-Hydride elimination

Transition

$\mathrm{ROTi}\left(\mathrm{N}\left(\mathrm{CH}_{3}\right)_{2}\right)-\mathrm{CH}_{2}---\mathrm{H}--\mathrm{N}\left(\mathrm{CH}_{3}\right)_{2} \mathrm{RO}$

$-3169.63875$

$\begin{array}{llll}\text { c } & 0.625717 & -3.144020 & 0.076697 \\ \mathrm{Si} & 2.311038 & -2.257831 & 0.272811 \\ \mathrm{Si} & 2.628223 & -0.712375 & 2.077406 \\ \mathrm{Si} & 3.575141 & 1.242129 & 1.137785 \\ \mathrm{Si} & 1.999048 & 2.293324 & -0.265239 \\ \mathrm{c} & 0.465596 & 2.993514 & 0.649625 \\ \mathrm{c} & -0.875706 & 2.978055 & -0.092753 \\ \mathrm{o} & -1.425257 & 1.692489 & -0.099394 \\ \mathrm{Ti} & -2.164546 & 0.048026 & -0.093139 \\ \mathrm{~h} & -3.261835 & -0.151115 & -1.555588 \\ \mathrm{c} & -3.956860 & -1.169192 & -0.803838 \\ \mathrm{c} & -0.480650 & -2.428210 & -0.693004 \\ \mathrm{o} & -0.867403 & -1.240092 & -0.040885 \\ \mathrm{n} & -3.295666 & -0.225855 & 1.650271 \\ \mathrm{c} & -4.339759 & 0.748785 & 1.942176 \\ \mathrm{c} & -2.643546 & -0.757152 & 2.839749 \\ \mathrm{Si} & 3.176693 & -1.083769 & -1.584204 \\ \mathrm{Si} & 1.772173 & 0.745986 & -2.077096 \\ \mathrm{c} & -3.586347 & 0.189050 & -2.916651 \\ \mathrm{~h} & 4.424043 & 0.566613 & 0.109016 \\ \mathrm{~h} & 3.260579 & -3.354898 & 0.594303 \\ \mathrm{~h} & 3.635931 & -1.345587 & 2.978542 \\ \mathrm{~h} & 2.666874 & 3.449750 & -0.924274 \\ \mathrm{~h} & 2.338368 & 1.437512 & -3.267454 \\ \mathrm{~h} & 1.390036 & -0.465130 & 2.873609 \\ \mathrm{~h} & 4.501315 & 2.089816 & 1.934237 \\ \mathrm{~h} & 3.787057 & -1.810637 & -2.729975 \\ \mathrm{~h} & 0.846089 & -4.090770 & -0.432670 \\ \mathrm{~h} & 0.257981 & -3.413296 & 1.073196 \\ \mathrm{~h} & 0.326459 & 2.472650 & 1.601701 \\ \mathrm{~h} & 0.725498 & 4.029025 & 0.901874 \\ \mathrm{~h} & -0.146043 & -2.189691 & -1.710710 \\ \mathrm{~h} & -1.352409 & -3.089041 & -0.789184 \\ \mathrm{~h} & -1.582466 & 3.656987 & 0.402838\end{array}$




$\begin{array}{llll}\text { h } & -0.766004 & 3.330208 & -1.127130 \\ \mathrm{~h} & 0.372227 & 0.361708 & -2.409540 \\ \mathrm{~h} & 4.297798 & -0.351291 & -0.900014 \\ \mathrm{~h} & -5.031523 & -0.957950 & -0.707360 \\ \mathrm{~h} & -3.801215 & -2.191678 & -1.170582 \\ \mathrm{~h} & -3.702562 & -0.986402 & 0.661837 \\ \mathrm{~h} & -4.628141 & 0.527336 & -3.000211 \\ \mathrm{~h} & -2.933792 & 0.991095 & -3.269464 \\ \mathrm{~h} & -3.463833 & -0.677216 & -3.581041 \\ \mathrm{~h} & -5.079582 & 0.340103 & 2.644374 \\ \mathrm{~h} & -3.930888 & 1.668695 & 2.380706 \\ \mathrm{~h} & -4.865383 & 1.023525 & 1.023117 \\ \mathrm{~h} & -3.382692 & -1.168912 & 3.540623 \\ \mathrm{~h} & -1.951764 & -1.553741 & 2.562373 \\ \mathrm{~h} & -2.071273 & 0.014786 & 3.373256\end{array}$

Adduct2 $\quad \mathrm{ROTi}\left(\mathrm{N}\left(\mathrm{CH}_{3}\right)_{2} 2_{2}-\mathrm{CH}_{2}-\underline{\mathrm{OR}}---\mathrm{HN}\left(\mathrm{CH}_{3}\right)_{2}\right.$ $-3169.534267$

$\begin{array}{llll}\text { c } & 0.918699 & -2.996047 & 1.079002 \\ \mathrm{Si} & 2.508215 & -1.978936 & 0.769119 \\ \mathrm{Si} & 2.834897 & 0.031654 & 2.026494 \\ \mathrm{Si} & 3.399044 & 1.725899 & 0.477158 \\ \mathrm{Si} & 1.550692 & 2.179176 & -0.910667 \\ \mathrm{c} & 0.055893 & 2.926286 & 0.023680 \\ \mathrm{c} & -1.332605 & 2.805413 & -0.624819 \\ \mathrm{o} & -1.870321 & 1.522548 & -0.493390 \\ \mathrm{Ti} & -2.125279 & -0.243546 & -0.117318 \\ \mathrm{C} & -3.946655 & -1.141976 & -0.646981 \\ \mathrm{n} & -2.887979 & -1.135304 & -1.583215 \\ \mathrm{c} & -3.108563 & -0.859757 & -2.984995 \\ \mathrm{c} & -0.331168 & -2.629343 & 0.279589 \\ \mathrm{o} & -0.758525 & -1.313300 & 0.553879 \\ \mathrm{n} & -2.955578 & 0.107214 & 1.961286 \\ \mathrm{c} & -4.113344 & 1.021834 & 2.014704 \\ \mathrm{c} & -1.907622 & 0.490868 & 2.930358 \\ \mathrm{Si} & 2.998139 & -1.301344 & -1.437150 \\ \mathrm{Si} & 1.326238 & 0.175425 & -2.190166 \\ \mathrm{~h} & 4.203450 & 0.870498 & -0.446758 \\ \mathrm{~h} & 3.625368 & -2.849013 & 1.226289 \\ \mathrm{~h} & 4.007922 & -0.226236 & 2.910516 \\ \mathrm{~h} & 1.978454 & 3.170605 & -1.938189 \\ \mathrm{~h} & 1.647137 & 0.560995 & -3.588929 \\ \mathrm{~h} & 1.673899 & 0.373181 & 2.902323 \\ \mathrm{~h} & 4.291572 & 2.844423 & 0.877176 \\ \mathrm{~h} & 3.559385 & -2.255930 & -2.427987 \\ \mathrm{~h} & 1.180045 & -4.039654 & 0.862040\end{array}$




$\begin{array}{llll}\text { h } & 0.690910 & -2.956898 & 2.150660 \\ \text { h } & 0.003342 & 2.503656 & 1.032123 \\ \text { h } & 0.301247 & 3.988407 & 0.153522 \\ \text { h } & -0.140409 & -2.744830 & -0.796068 \\ \text { h } & -1.134008 & -3.337686 & 0.528051 \\ \text { h } & -2.015023 & 3.524619 & -0.148113 \\ \text { h } & -1.289509 & 3.077463 & -1.688518 \\ \text { h } & -0.040335 & -0.413487 & -2.194748 \\ \text { h } & 4.082459 & -0.301085 & -1.146511 \\ \text { h } & -4.812161 & -0.509996 & -0.901017 \\ \text { h } & -4.262830 & -2.117820 & -0.256351 \\ \text { h } & -3.284375 & -0.824533 & 2.199097 \\ \text { h } & -3.802925 & -0.016920 & -3.132006 \\ \text { h } & -2.165616 & -0.614494 & -3.481324 \\ \text { h } & -3.539346 & -1.731282 & -3.495250 \\ \text { h } & -4.501455 & 1.126692 & 3.035128 \\ \text { h } & -3.807505 & 2.002865 & 1.650875 \\ \text { h } & -4.897117 & 0.642011 & 1.362130 \\ \text { h } & -2.290401 & 0.515004 & 3.957487 \\ \text { h } & -1.082493 & -0.214750 & 2.857272 \\ \text { h } & -1.536873 & 1.484934 & 2.677414\end{array}$

Product $\quad \mathrm{ROTi}\left(\mathrm{N}\left(\mathrm{CH}_{3}\right)_{2}\right)-\mathrm{CH}_{2}-\underline{-O R} \quad-3034.303748$

$\begin{array}{llll}\text { c } & -0.794283 & -3.105219 & -0.963103 \\ \text { c } & 0.651657 & -2.727747 & -0.651795 \\ \text { o } & 0.927188 & -1.383166 & -0.984059 \\ \mathrm{Ti} & 2.193419 & -0.137003 & -0.520065 \\ \mathrm{Si} & -2.178148 & -2.101695 & -0.102651 \\ \mathrm{Si} & -1.798873 & -1.083203 & 1.995230 \\ \mathrm{Si} & -0.128037 & 0.556102 & 1.785597 \\ \mathrm{Si} & -0.995421 & 2.308618 & 0.411968 \\ \mathrm{c} & -0.036651 & 2.834592 & -1.154505 \\ \mathrm{c} & 1.490350 & 2.908684 & -1.022844 \\ \mathrm{o} & 2.054506 & 1.643496 & -0.819284 \\ \mathrm{Si} & -3.156227 & -0.333322 & -1.379644 \\ \mathrm{Si} & -3.195990 & 1.581444 & -0.000939 \\ \mathrm{c} & 4.104591 & -0.833309 & -0.803142 \\ \mathrm{n} & 3.621642 & -0.752980 & 0.534784 \\ \mathrm{c} & 4.426260 & -0.151039 & 1.578335 \\ \mathrm{~h} & -3.473734 & 0.886789 & 1.291283 \\ \mathrm{~h} & -3.302780 & -3.052162 & 0.098261 \\ \mathrm{~h} & -4.557129 & -0.761124 & -1.645183 \\ \mathrm{~h} & -1.042885 & 3.446058 & 1.374290 \\ \mathrm{~h} & 0.150134 & 1.161359 & 3.106829 \\ \mathrm{~h} & -2.472535 & -0.121806 & -2.688947\end{array}$




$\begin{array}{llll}\text { h } & -4.258583 & 2.605009 & -0.153941 \\ \mathrm{~h} & -1.817880 & -1.845365 & 3.268204 \\ \mathrm{~h} & -0.950334 & -4.156053 & -0.691426 \\ \mathrm{~h} & -0.959138 & -3.039850 & -2.044280 \\ \mathrm{~h} & -0.282802 & 2.154418 & -1.976791 \\ \mathrm{~h} & -0.428343 & 3.818730 & -1.441457 \\ \mathrm{~h} & 0.864783 & -2.902083 & 0.412143 \\ \mathrm{~h} & 1.327830 & -3.379875 & -1.219754 \\ \mathrm{~h} & 1.916560 & 3.338974 & -1.938502 \\ \mathrm{~h} & 1.777594 & 3.570621 & -0.194834 \\ \mathrm{~h} & 1.211864 & -0.003087 & 1.379207 \\ \mathrm{~h} & -2.980076 & -0.146433 & 2.043411 \\ \mathrm{~h} & 4.884994 & -0.112417 & -1.079550 \\ \mathrm{~h} & 4.285163 & -1.832910 & -1.206745 \\ \mathrm{~h} & 4.926597 & 0.769466 & 1.238831 \\ \mathrm{~h} & 3.800688 & 0.099258 & 2.440548 \\ \mathrm{~h} & 5.205953 & -0.842226 & 1.921545\end{array}$

TDMAT deposition on -SH SAM on diagonal dimer substrate (Figure 13)

Reactant

$\begin{array}{llll}\text { c } & -2.851421 & 1.030004 & 0.382002 \\ \text { SI } & -2.325584 & -0.781845 & 0.710823 \\ \text { SI } & -0.498792 & -1.071469 & 2.229402 \\ \text { SI } & 1.046969 & -2.494578 & 1.155496 \\ \text { SI } & 1.978866 & -1.405762 & -0.718645 \\ \text { c } & 3.019673 & 0.176807 & -0.441988 \\ \text { c } & 2.302007 & 1.361536 & 0.197208 \\ \text { S } & 3.477865 & 2.785192 & 0.338135 \\ \text { c } & -1.773040 & 1.968134 & -0.151357 \\ \text { S } & -2.491950 & 3.660094 & -0.375416 \\ \text { Si } & -1.732023 & -2.139270 & -1.122858 \\ \text { SI } & 0.133118 & -1.208909 & -2.214541 \\ \text { h } & 0.044895 & -3.363146 & 0.455726 \\ \text { h } & -3.469960 & -1.424023 & 1.405231 \\ \text { h } & -1.077260 & -1.760115 & 3.410706 \\ \text { h } & 2.869924 & -2.367665 & -1.415162 \\ \text { h } & 0.475956 & -2.069197 & -3.372546 \\ \text { h } & 0.080358 & 0.226340 & 2.688092 \\ \text { h } & 1.894151 & -3.414870 & 1.953001 \\ \text { h } & -2.761928 & -2.779778 & -1.976825 \\ \text { h } & -3.699399 & 1.003010 & -0.311340 \\ \text { h } & -3.245730 & 1.416270 & 1.328704\end{array}$




$\begin{array}{llll}\text { h } & 3.886874 & -0.106899 & 0.164594 \\ \text { h } & 3.416604 & 0.465884 & -1.421783 \\ \text { h } & -0.938455 & 2.036136 & 0.548231 \\ \text { h } & -1.395427 & 1.624661 & -1.115003 \\ \text { h } & -1.386475 & 4.239539 & -0.897895 \\ \text { h } & 1.451290 & 1.673334 & -0.410974 \\ \text { h } & 1.943480 & 1.110981 & 1.195970 \\ \text { h } & 2.636955 & 3.628859 & 0.979861 \\ \text { h } & -0.082381 & 0.183591 & -2.708862 \\ \text { h } & -1.037171 & -3.240710 & -0.373890\end{array}$

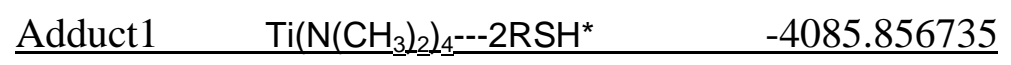

\begin{tabular}{|c|c|c|c|}
\hline C & -2.520664 & 3.015287 & 0.435041 \\
\hline $\mathrm{Si}$ & -4.191692 & 2.129701 & 0.730024 \\
\hline $\mathrm{Si}$ & -4.179415 & 0.266448 & 2.234653 \\
\hline $\mathrm{Si}$ & -5.282339 & -1.498989 & 1.12291 \\
\hline $\mathrm{Si}$ & -4.002882 & -2.211313 & -0.72827 \\
\hline $\mathrm{C}$ & -2.280948 & -2.984639 & -0.414981 \\
\hline C & -1.196421 & -2.077245 & 0.159158 \\
\hline$S$ & 0.354032 & -3.061796 & 0.393468 \\
\hline C & -1.364014 & 2.173894 & -0.096052 \\
\hline S & 0.110284 & 3.263547 & -0.35419 \\
\hline $\mathrm{Si}$ & -5.382317 & 1.312856 & -1.135267 \\
\hline $\mathrm{Si}$ & -4.108701 & -0.346444 & -2.211942 \\
\hline $\mathrm{n}$ & 2.766023 & 0.663769 & -1.519839 \\
\hline $\mathrm{Ti}$ & 3.838031 & 0.022387 & -0.033906 \\
\hline $\mathrm{n}$ & 5.081719 & 1.370309 & 0.495736 \\
\hline C & 5.154351 & 2.115262 & 1.739418 \\
\hline C & 1.714935 & -0.156015 & -2.106509 \\
\hline C & 3.14546 & 1.750822 & -2.414791 \\
\hline $\mathrm{n}$ & 2.795479 & -0.435763 & 1.534766 \\
\hline C & 3.202689 & -1.445392 & 2.505109 \\
\hline $\mathrm{n}$ & 4.846797 & -1.533051 & -0.527199 \\
\hline C & 6.128613 & -1.860143 & 0.079423 \\
\hline C & 1.76604 & 0.438757 & 2.078076 \\
\hline C & 4.468188 & -2.506509 & -1.538466 \\
\hline C & 6.18127 & 1.688492 & -0.40444 \\
\hline h & -6.303466 & -0.663902 & 0.410039 \\
\hline h & -5.049494 & 3.129079 & 1.413449 \\
\hline h & -4.993962 & 0.697767 & 3.40044 \\
\hline h & -4.780517 & -3.248921 & -1.45168 \\
\hline h & -4.873775 & -0.823579 & -3.390154 \\
\hline h & -2.811317 & -0.078169 & 2.724003 \\
\hline h & -6.054497 & -2.504373 & 1.896003 \\
\hline h & -6.182594 & 2.221735 & -1.992994 \\
\hline
\end{tabular}




$\begin{array}{llll}\mathrm{h} & -2.724783 & 3.845827 & -0.250809 \\ \mathrm{~h} & -2.234441 & 3.475866 & 1.386966 \\ \mathrm{~h} & -2.43667 & -3.845588 & 0.245486 \\ \mathrm{~h} & -1.94504 & -3.398613 & -1.372141 \\ \mathrm{~h} & -1.095683 & 1.391816 & 0.615996 \\ \mathrm{~h} & -1.620225 & 1.705236 & -1.047018 \\ \mathrm{~h} & 1.001644 & 2.283961 & -0.643639 \\ \mathrm{~h} & -0.983771 & -1.251184 & -0.521293 \\ \mathrm{~h} & -1.496259 & -1.663596 & 1.123013 \\ \mathrm{~h} & 1.182145 & -2.029983 & 0.702534 \\ \mathrm{~h} & -2.771097 & 0.125666 & -2.678296 \\ \mathrm{~h} & -6.358676 & 0.4304-0.412066 \\ \mathrm{~h} & 6.099349 & -2.840862 & 0.578686 \\ \mathrm{~h} & 6.933943 & -1.903749 & -0.670188 \\ \mathrm{~h} & 6.404975 & -1.113975 & 0.826325 \\ \mathrm{~h} & 4.392848 & -3.518833 & -1.113798 \\ \mathrm{~h} & 3.49999 & -2.264379 & -1.979438 \\ \mathrm{~h} & 5.204845 & -2.550175 & -2.355776 \\ \mathrm{~h} & 6.090616 & 1.90721 & 2.280725 \\ \mathrm{~h} & 5.115823 & 3.200893 & 1.564045 \\ \mathrm{~h} & 4.323663 & 1.85168 & 2.394626 \\ \mathrm{~h} & 7.157184 & 1.490592 & 0.06418 \\ \mathrm{~h} & 6.128413 & 1.086014 & -1.315735 \\ \mathrm{~h} & 6.169838 & 2.746963 & -0.704396 \\ \mathrm{~h} & 2.280899 & 2.377237 & -2.679346 \\ \mathrm{~h} & 3.886573 & 2.397756 & -1.944084 \\ \mathrm{~h} & 3.568434 & 1.37501 & -3.359703 \\ \mathrm{~h} & 0.806191 & 0.433339 & -2.304488 \\ \mathrm{~h} & 2.021772 & -0.596411 & -3.068142 \\ \mathrm{~h} & 1.436638 & -0.979097 & -1.4445 \\ \mathrm{~h} & 2.348542 & -2.048307 & 2.847268 \\ \mathrm{~h} & 3.933054 & -2.123221 & 2.06313 \\ \mathrm{~h} & 3.653573 & -0.989313 & 3.400072 \\ \mathrm{~h} & 1.487035 & 1.212165 & 1.361094 \\ \mathrm{~h} & 0.856326 & -0.122088 & 2.344447 \\ & 2.101917 & 0.947704 & 2.994974\end{array}$

$\begin{array}{lll}\text { Transition } & \mathrm{Ti}\left(\mathrm{N}\left(\mathrm{CH}_{3}\right)_{2}\right)_{2} 2_{4}--\mathrm{H}_{-}--\mathrm{SRRSH}^{*} & -4085.848348\end{array}$

$\begin{array}{llll}\text { C } & -1.819284 & -2.603355 & -1.040815 \\ \mathrm{Si} & -3.694561 & -2.452161 & -0.713939 \\ \mathrm{Si} & -4.803434 & -0.700368 & -1.910914 \\ \mathrm{Si} & -6.041042 & 0.540377 & -0.331244 \\ \mathrm{Si} & -4.576565 & 1.641242 & 1.156934 \\ \mathrm{C} & -3.37358 & 2.959381 & 0.459552\end{array}$




\begin{tabular}{|c|c|c|c|}
\hline C & -2.410126 & 2.483272 & -0.622609 \\
\hline S & -1.310967 & 3.883691 & -1.13755 \\
\hline C & -0.952748 & -1.40994 & -0.636438 \\
\hline S & 0.815887 & -1.660221 & -1.11255 \\
\hline $\mathrm{Si}$ & -4.466579 & -2.14517 & 1.495219 \\
\hline $\mathrm{Si}$ & -3.575302 & -0.150231 & 2.367429 \\
\hline $\mathrm{n}$ & 2.376488 & -0.292811 & 1.046739 \\
\hline $\mathrm{Ti}$ & 4.166503 & -0.000851 & 0.056224 \\
\hline $\mathrm{n}$ & 4.476538 & -1.434162 & -1.138363 \\
\hline C & 4.48746 & -1.426132 & -2.594942 \\
\hline C & 1.612162 & 0.900677 & 1.441135 \\
\hline C & 2.374637 & -1.286407 & 2.131046 \\
\hline $\mathrm{n}$ & 4.057647 & 1.643682 & -0.869657 \\
\hline C & 4.865546 & 2.845392 & -0.748382 \\
\hline $\mathrm{n}$ & 5.578693 & 0.077635 & 1.322767 \\
\hline C & 6.789113 & -0.719165 & 1.433534 \\
\hline C & 2.995585 & 1.778085 & -1.863977 \\
\hline C & 5.445435 & 1.041852 & 2.407209 \\
\hline C & 4.546392 & -2.789179 & -0.599131 \\
\hline h & -6.440694 & -0.611347 & 0.543172 \\
\hline h & -4.346447 & -3.683432 & -1.229411 \\
\hline h & -5.744468 & -1.377834 & -2.839133 \\
\hline h & -5.392415 & 2.331344 & 2.189224 \\
\hline h & -4.044517 & 0.01776 & 3.764464 \\
\hline h & -3.870889 & 0.128428 & -2.731386 \\
\hline h & -7.309777 & 1.208764 & -0.717601 \\
\hline h & -4.601304 & -3.281073 & 2.442591 \\
\hline h & -1.455709 & -3.506872 & -0.539486 \\
\hline h & -1.702048 & -2.79391 & -2.113827 \\
\hline h & -3.980975 & 3.788623 & 0.080451 \\
\hline h & -2.813196 & 3.357964 & 1.313182 \\
\hline h & -1.319004 & -0.49969 & -1.118813 \\
\hline h & -1.011166 & -1.254044 & 0.442578 \\
\hline h & 1.616763 & -0.889617 & 0.070706 \\
\hline h & -1.780684 & 1.670022 & -0.259888 \\
\hline h & -2.951448 & 2.134719 & -1.502022 \\
\hline h & -0.688906 & 3.233794 & -2.148079 \\
\hline h & -2.083441 & -0.088697 & 2.376526 \\
\hline h & -5.860508 & -1.659312 & 1.20763 \\
\hline h & 7.685408 & -0.082342 & 1.419483 \\
\hline h & 6.812036 & -1.294868 & 2.371014 \\
\hline h & 6.864129 & -1.417464 & 0.599842 \\
\hline h & 6.295028 & 1.739286 & 2.432709 \\
\hline $\mathrm{h}$ & 4.537139 & 1.642335 & 2.291073 \\
\hline h & 5.39267 & \multicolumn{2}{|c|}{0.54843 .38817} \\
\hline h & 5.396211 & -1.90991 & -2.979842 \\
\hline
\end{tabular}




$\begin{array}{llll}\text { h } & 3.61917 & -1.960925 & -3.000991 \\ \mathrm{~h} & 4.467633 & -0.404092 & -2.973391 \\ \mathrm{~h} & 5.454873 & -3.301974 & -0.945003 \\ \mathrm{~h} & 4.581918 & -2.777064 & 0.494617 \\ \mathrm{~h} & 3.676905 & -3.386307 & -0.89994 \\ \mathrm{~h} & 1.347365 & -1.534027 & 2.4259 \\ \mathrm{~h} & 2.843552 & -2.213152 & 1.798344 \\ \mathrm{~h} & 2.905344 & -0.924275 & 3.019096 \\ \mathrm{~h} & 0.590397 & 0.625177 & 1.731843 \\ \mathrm{~h} & 2.067088 & 1.415992 & 2.296402 \\ \mathrm{~h} & 1.548241 & 1.605426 & 0.612472 \\ \mathrm{~h} & 4.258131 & 3.709528 & -0.442025 \\ \mathrm{~h} & 5.657012 & 2.702018 & -0.012316 \\ \mathrm{~h} & 5.339815 & 3.101958 & -1.706387 \\ \mathrm{~h} & 2.381282 & 0.873243 & -1.922819 \\ \mathrm{~h} & 2.325834 & 2.613705 & -1.615725 \\ \mathrm{~h} & 3.407507 & 1.967995 & -2.865025\end{array}$

Adduct2 $\mathrm{Ti}\left(\mathrm{N}_{\left(\mathrm{CH}_{3}\right.} 2_{2} 2_{3}{ }_{3}--\mathrm{SRRSH}^{*}--\mathrm{HN}\left(\mathrm{CH}_{3}\right)_{2} 2_{4}\right.$ $-4085.877723$

$\begin{array}{llll}\text { c } & -0.802765 & -2.427476 & -1.015494 \\ \mathrm{Si} & -2.713253 & -2.520861 & -1.047435 \\ \mathrm{Si} & -3.986487 & -0.847887 & -2.205227 \\ \mathrm{Si} & -5.7182 & -0.169717 & -0.7447 \\ \mathrm{Si} & -4.80597 & 0.947902 & 1.121065 \\ \mathrm{c} & -3.921789 & 2.621107 & 0.832301 \\ \mathrm{c} & -2.789819 & 2.652325 & -0.188087 \\ \mathrm{~S} & -2.160749 & 4.387674 & -0.337117 \\ \mathrm{c} & -0.079234 & -1.301484 & -0.278503 \\ \mathrm{~S} & 1.724707 & -1.71389 & -0.167585 \\ \mathrm{Ti} & 3.236672 & 0.129431 & -0.013944 \\ \mathrm{n} & 1.96271 .579317 & -0.152722 \\ \mathrm{C} & 1.399871 & 2.353421 & 0.941527 \\ \mathrm{n} & 3.975687 & 0.624896 & 1.691286 \\ \mathrm{C} & 4.882664 & 1.735354 & 1.912004 \\ \mathrm{n} & 4.766858 & -1.875443 & 0.079591 \\ \mathrm{c} & 4.570679 & -2.894025 & 1.130585 \\ \mathrm{n} & 4.219906 & 0.56764 & -1.622176 \\ \mathrm{c} & 4.387288 & -0.318414 & -2.762324 \\ \mathrm{C} & 3.52523 & 0.040668 & 2.94503 \\ \mathrm{Si} & -3.881676 & -2.742309 & 0.992963 \\ \mathrm{Si} & -3.616708 & -0.777838 & 2.264877 \\ \mathrm{C} & 6.152118 & -1.370664 & 0.111341 \\ \mathrm{c} & 4.727246 & 1.898132 & -1.908633 \\ \mathrm{c} & 1.357814 & 1.970957 & -1.41598 \\ \mathrm{~h} & -5.976647 & -1.507397 & -0.119975\end{array}$




\begin{tabular}{|c|c|c|c|}
\hline h & -2.979011 & -3.748884 & -1.839354 \\
\hline h & -4.594935 & -1.537611 & -3.37611 \\
\hline h & -5.907001 & 1.26734 & 2.062314 \\
\hline $\mathrm{h}$ & -4.33304 & -0.955872 & 3.5545 \\
\hline $\mathrm{h}$ & -3.155116 & 0.281787 & -2.71879 \\
\hline l & -7.028786 & 0.283927 & -1.278225 \\
\hline l & -3.900493 & -4.029236 & 1.735722 \\
\hline l & -0.497347 & -3.389705 & -0.587163 \\
\hline l & -0.463656 & -2.458547 & -2.056772 \\
\hline $\mathrm{l}$ & -4.70653 & 3.327266 & 0.53686 \\
\hline h & -3.557342 & 2.966637 & 1.806231 \\
\hline l & -0.204066 & -0.347491 & -0.79054 \\
\hline l & -0.451441 & -1.188563 & 0.740973 \\
\hline l & 4.622749 & -2.337443 & -0.81435 \\
\hline l & -1.965101 & 2.008331 & 0.122256 \\
\hline l & -3.133659 & 2.326174 & -1.169756 \\
\hline l & -1.185572 & 4.131171 & -1.238398 \\
\hline l & -2.196222 & -0.426634 & 2.56052 \\
\hline l & -5.284617 & -2.512401 & 0.502596 \\
\hline l & 4.402188 & 2.552129 & 2.473645 \\
\hline l & 5.767898 & 1.425764 & 2.489281 \\
\hline $\mathrm{h}$ & 5.239577 & 2.147895 & 0.966759 \\
\hline l & 3.023114 & 0.785715 & 3.582512 \\
\hline $\mathrm{h}$ & \multicolumn{3}{|c|}{$2.8178-0.77207$} \\
\hline $\mathrm{h}$ & 4.365932 & -0.363673 & 3.530798 \\
\hline $\mathrm{l}$ & 3.951113 & 0.117409 & -3.674514 \\
\hline $\mathrm{l}$ & 5.44872 & -0.518984 & -2.985484 \\
\hline $\mathrm{h}$ & 3.885173 & -1.274237 & -2.599997 \\
\hline $\mathrm{l}$ & 4.29611 & 2.305009 & -2.836732 \\
\hline l & 4.477497 & 2.589666 & -1.102974 \\
\hline $\mathrm{l}$ & 5.82176 & 1.903503 & -2.034232 \\
\hline l & 6.888276 & -2.182668 & 0.045076 \\
\hline l & 6.306455 & -0.670786 & -0.707036 \\
\hline $\mathrm{l}$ & 6.307356 & -0.835581 & 1.04835 \\
\hline $\mathrm{l}$ & 5.308703 & -3.703456 & 1.054742 \\
\hline $\mathrm{h}$ & 4.680077 & -2.423992 & 2.108088 \\
\hline $\mathrm{h}$ & 3.568268 & -3.310788 & 1.056009 \\
\hline $\mathrm{h}$ & \multicolumn{3}{|c|}{$0.32072 .172413 \quad 1.062059$} \\
\hline $\mathrm{h}$ & 1.887132 & 2.104684 & 1.882379 \\
\hline $\mathrm{h}$ & 1.517417 & 3.434501 & 0.768555 \\
\hline $\mathrm{l}$ & 1.78061 & 1.403182 & -2.244348 \\
\hline $\mathrm{l}$ & 0.268406 & 1.807196 & -1.419542 \\
\hline & 1.51768 & 3.041947 & -1.61864 \\
\hline
\end{tabular}




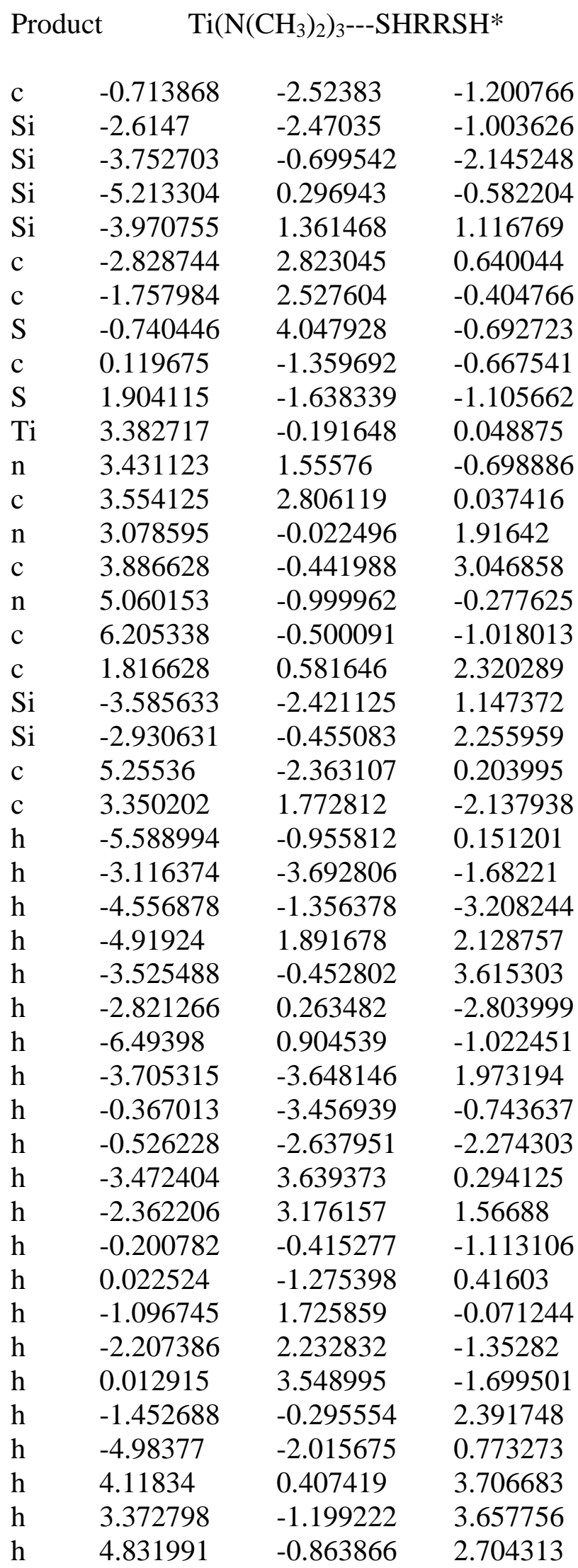




$\begin{array}{llll}\text { h } & 1.978739 & 1.45909 & 2.96312 \\ \text { h } & 1.250552 & 0.924656 & 1.44706 \\ \text { h } & 1.179486 & -0.124147 & 2.871373 \\ \text { h } & 7.111588 & -0.497952 & -0.394576 \\ \text { h } & 6.415564 & -1.12313 & -1.899513 \\ \text { h } & 6.02323 & 0.520489 & -1.353254 \\ \text { h } & 6.118766 & -2.427423 & 0.88177 \\ \text { h } & 4.374779 & -2.717938 & 0.746449 \\ \text { h } & 5.425431 & -3.059714 & -0.628171 \\ \text { h } & 2.682923 & 3.459064 & -0.117731 \\ \text { h } & 3.648856 & 2.610214 & 1.105382 \\ \text { h } & 4.445281 & 3.365994 & -0.282829 \\ \text { h } & 3.246752 & 0.82696 & -2.672774 \\ \text { h } & 2.484213 & 2.398676 & -2.401797 \\ \text { h } & 4.248452 & 2.282916 & -2.516474\end{array}$

Transition RS---H---Ti( $\left(\mathrm{N}\left(\mathrm{CH}_{3}\right)_{2} 2_{3} \underline{\mathrm{SR}}\right.$

$-3950.647331$

$\begin{array}{llll}\text { c } & -0.996470 & -2.890114 & 0.030645 \\ \text { SI } & -2.765499 & -2.482389 & -0.583470 \\ \text { SI } & -3.041902 & -0.773839 & -2.245511 \\ \text { SI } & -4.664615 & 0.716451 & -1.386531 \\ \text { SI } & -3.792755 & 1.868185 & 0.481346 \\ \text { c } & -2.232768 & 2.948300 & 0.284603 \\ \text { c } & -0.952498 & 2.192784 & -0.084324 \\ \text { S } & 0.536258 & 3.278271 & 0.011535 \\ \text { Ti } & 3.105536 & -0.252676 & 0.042527 \\ \text { n } & 3.107309 & -0.046050 & 1.904265 \\ \text { c } & 3.985662 & -0.644930 & 2.898846 \\ \text { c } & -0.076301 & -1.707275 & 0.406539 \\ \text { S } & 1.439933 & -1.691294 & -0.670060 \\ \text { n } & 4.660199 & -1.147232 & -0.524439 \\ \text { c } & 4.900338 & -2.522620 & -0.094813 \\ \text { n } & 2.851228 & 1.588975 & -0.862494 \\ \text { c } & 3.872955 & 2.587873 & -0.459686 \\ \text { c } & 5.656664 & -0.682731 & -1.478055 \\ \text { Si } & -4.303297 & -1.863526 & 1.084184 \\ \text { Si } & -3.592096 & 0.132999 & 2.097534 \\ \text { c } & 2.665832 & 1.577707 & -2.335489 \\ \text { c } & 2.056275 & 0.779264 & 2.507267 \\ \text { h } & -5.509628 & -0.321676 & -0.701613 \\ \text { h } & -3.263245 & -3.718331 & -1.242870 \\ \text { h } & -3.597254 & -1.474477 & -3.436202 \\ \text { h } & -4.891042 & 2.684340 & 1.067709 \\ \text { h } & -4.570075 & 0.489214 & 3.152263 \\ \text { h } & -1.754651 & -0.136464 & -2.646728\end{array}$




$\begin{array}{llll}\text { h } & -5.585879 & 1.430676 & -2.308439 \\ \mathrm{~h} & -4.934267 & -2.882720 & 1.953985 \\ \mathrm{~h} & -1.115395 & -3.559710 & 0.892461 \\ \mathrm{~h} & -0.527009 & -3.498305 & -0.746596 \\ \mathrm{~h} & -2.423877 & 3.737886 & -0.449497 \\ \mathrm{~h} & -2.085396 & 3.461134 & 1.241849 \\ \mathrm{~h} & -0.564621 & -0.741102 & 0.288421 \\ \mathrm{~h} & 0.248328 & -1.781543 & 1.443945 \\ \mathrm{~h} & -0.824317 & 1.349917 & 0.603367 \\ \mathrm{~h} & -1.042402 & 1.778120 & -1.092061 \\ \mathrm{~h} & 1.871563 & 2.102953 & -0.481876 \\ \mathrm{~h} & -2.240954 & 0.024264 & 2.727392 \\ \mathrm{~h} & -5.380463 & -1.316033 & 0.197520 \\ \mathrm{~h} & 4.468032 & 0.131480 & 3.507182 \\ \mathrm{~h} & 3.425821 & -1.299041 & 3.580756 \\ \mathrm{~h} & 4.769912 & -1.233225 & 2.422723 \\ \mathrm{~h} & 2.497726 & 1.553068 & 3.147213 \\ \mathrm{~h} & 1.464563 & 1.310500 & 1.754450 \\ \mathrm{~h} & 1.381415 & 0.172672 & 3.124031 \\ \mathrm{~h} & 4.877858 & -3.207678 & -0.951949 \\ \mathrm{~h} & 5.878360 & -2.616528 & 0.395672 \\ \mathrm{~h} & 4.133473 & -2.856507 & 0.605955 \\ \mathrm{~h} & 5.671813 & -1.314957 & -2.375832 \\ \mathrm{~h} & 5.453501 & 0.341820 & -1.794171 \\ \mathrm{~h} & 6.662874 & -0.701316 & -1.038507 \\ \mathrm{~h} & 3.554525 & 3.584577 & -0.779823 \\ \mathrm{~h} & 3.971874 & 2.596308 & 0.625653 \\ \mathrm{~h} & 4.844591 & 2.367794 & -0.908123 \\ \mathrm{~h} & 1.884624 & 0.865879 & -2.599844 \\ \mathrm{~h} & 2.351997 & 2.571530 & -2.667319 \\ \mathrm{~h} & 3.589759 & 1.302993 & -2.850524 \\ & & & \\ & & & \\ & & & \end{array}$

Adduct2 $\left.\quad\left(\mathrm{CH}_{3}\right)_{2}\right) \mathrm{NH}---\mathrm{RS}---\mathrm{Ti}\left(\mathrm{N}\left(\mathrm{CH}_{3}\right)_{2}\right)_{2} \mathrm{SR}$

$-3950.692773$

$\begin{array}{llll}\mathrm{c} & 1.536482 & -2.930232 & -0.340165 \\ \text { SI } & 3.120458 & -2.169673 & 0.428837 \\ \text { SI } & 2.927384 & -0.536755 & 2.177811 \\ \text { SI } & 4.222100 & 1.321072 & 1.495113 \\ \text { SI } & 3.240442 & 2.335500 & -0.393452 \\ \text { c } & 1.407474 & 2.876950 & -0.299815 \\ \text { c } & 0.528408 & 1.733827 & 0.212936 \\ \text { S } & -1.249182 & 1.918194 & -0.268383 \\ \mathrm{Ti} & -2.479231 & -0.114757 & 0.000742 \\ \mathrm{n} & -2.971698 & -0.990943 & -1.625488 \\ \mathrm{c} & -3.335203 & -2.393573 & -1.752599 \\ \mathrm{c} & 0.382372 & -2.011958 & -0.779728\end{array}$




\begin{tabular}{|c|c|c|c|}
\hline$S$ & -0.704839 & -1.553554 & 0.646745 \\
\hline$n$ & -3.346422 & -0.547943 & 1.642396 \\
\hline c & -4.038359 & -1.793611 & 1.925871 \\
\hline $\mathrm{n}$ & -4.232225 & 1.519852 & -0.450835 \\
\hline C & -5.490866 & 0.805547 & -0.743586 \\
\hline C & -3.005763 & 0.170739 & 2.865230 \\
\hline Si & 4.595425 & -1.133387 & -1.102035 \\
\hline $\mathrm{Si}$ & 3.523864 & 0.701409 & -2.089341 \\
\hline $\mathrm{C}$ & -4.459336 & 2.539970 & 0.595024 \\
\hline C & -2.901024 & -0.337010 & -2.926120 \\
\hline h & 5.318914 & 0.556873 & 0.811900 \\
\hline h & 3.890140 & -3.287465 & 1.054015 \\
\hline h & 3.574994 & -1.156137 & 3.359428 \\
\hline h & 4.122610 & 3.439805 & -0.863816 \\
\hline h & 4.433534 & 1.352351 & -3.057734 \\
\hline h & 1.534711 & -0.195686 & 2.561297 \\
\hline h & 4.899301 & 2.185377 & 2.494186 \\
\hline h & 5.498494 & -1.911337 & -1.984216 \\
\hline h & 1.885865 & -3.499399 & -1.212178 \\
\hline $\mathrm{h}$ & 1.153345 & -3.676159 & 0.363485 \\
\hline h & 1.280114 & 3.772180 & 0.315891 \\
\hline h & 1.111777 & 3.156055 & -1.316590 \\
\hline h & 0.751069 & -1.111489 & -1.276028 \\
\hline h & -0.243620 & -2.533066 & -1.507042 \\
\hline h & 0.860919 & 0.772808 & -0.175037 \\
\hline h & 0.574512 & 1.669122 & 1.298821 \\
\hline h & -3.945572 & 2.012420 & -1.293696 \\
\hline h & 2.251465 & 0.365588 & -2.796467 \\
\hline h & 5.472861 & -0.387457 & -0.135963 \\
\hline h & -4.369999 & -2.514083 & -2.108626 \\
\hline h & -2.682998 & -2.909779 & -2.471466 \\
\hline h & -3.240907 & -2.912056 & -0.798293 \\
\hline h & -3.879469 & -0.328478 & -3.432812 \\
\hline h & -2.551230 & 0.692285 & -2.833168 \\
\hline h & -2.198589 & -0.856525 & -3.592748 \\
\hline h & -3.398473 & -2.487164 & 2.489940 \\
\hline h & -4.945890 & -1.612368 & 2.519465 \\
\hline h & -4.343380 & -2.293152 & 1.005036 \\
\hline h & -2.375376 & -0.444335 & 3.522141 \\
\hline h & -2.458171 & 1.088281 & 2.641221 \\
\hline h & -3.908753 & 0.445248 & 3.429827 \\
\hline h & -6.306356 & 1.499245 & -0.983166 \\
\hline h & -5.343473 & 0.118725 & -1.573560 \\
\hline h & -5.775619 & 0.225774 & 0.135116 \\
\hline h & -3.544380 & 3.105453 & 0.757897 \\
\hline h & -5.268941 & 3.226623 & 0.318651 \\
\hline
\end{tabular}




$\begin{array}{llll}\text { h } & -4.734149 & 2.037359 & 1.522309\end{array}$

Product

$\left.\mathrm{RSTi}\left(\mathrm{N}_{\left(\mathrm{CH}_{3}\right.}\right)_{2}\right)_{2}{ }_{2} \mathrm{SR}_{-}-3815.483174$

$\begin{array}{llll}\text { c } & 0.940228 & 3.046103 & 0.188678 \\ \mathrm{c} & -0.251378 & 2.178241 & 0.619054 \\ \mathrm{~S} & -1.257550 & 1.617457 & -0.842335 \\ \mathrm{Ti} & -2.706616 & -0.077111 & -0.090778 \\ \mathrm{SI} & 2.583766 & 2.253767 & -0.406559 \\ \mathrm{SI} & 3.931454 & 1.210609 & 1.236381 \\ \mathrm{SI} & 2.813404 & -0.670277 & 2.084965 \\ \mathrm{SI} & 2.725841 & -2.275471 & 0.334488 \\ \mathrm{c} & 0.941687 & -2.883606 & -0.014599 \\ \mathrm{c} & 0.009124 & -1.685676 & -0.168480 \\ \mathrm{~S} & -1.751144 & -2.137047 & -0.579689 \\ \mathrm{Si} & 2.591195 & 0.648533 & -2.194573 \\ \mathrm{Si} & 3.858934 & -1.197403 & -1.428472 \\ \mathrm{~h} & -3.006776 & 0.070888 & 1.760774 \\ \mathrm{c} & -2.740850 & -0.834417 & 2.868787 \\ \mathrm{~h} & -4.343272 & -0.059505 & -1.010696 \\ \mathrm{c} & -4.262853 & 0.008382 & -2.467283 \\ \mathrm{c} & -3.638701 & 1.317254 & 2.186753 \\ \mathrm{c} & -5.708483 & -0.070482 & -0.515253 \\ \mathrm{~h} & 4.868359 & -0.425219 & -0.633402 \\ \mathrm{~h} & 3.385293 & 3.394328 & -0.934129 \\ \mathrm{~h} & 3.332127 & 1.306314 & -3.299339 \\ \mathrm{~h} & 3.584190 & -3.366011 & 0.867674 \\ \mathrm{~h} & 3.644485 & -1.319603 & 3.120670 \\ \mathrm{~h} & 1.241776 & 0.288327 & -2.704626 \\ \mathrm{~h} & 4.642376 & -2.025834 & -2.378441 \\ \mathrm{~h} & 4.733666 & 1.986469 & 2.214078 \\ \mathrm{~h} & 1.232159 & 3.674088 & 1.041379 \\ \mathrm{~h} & 0.617412 & 3.745014 & -0.589438 \\ \mathrm{~h} & 0.925370 & -3.500886 & -0.917984 \\ \mathrm{~h} & 0.615643 & -3.524804 & 0.810451 \\ \mathrm{~h} & 0.073275 & 1.313679 & 1.199667 \\ \mathrm{~h} & -0.915894 & 2.763353 & 1.256848 \\ \mathrm{~h} & 0.011464 & -1.102951 & 0.754800 \\ \mathrm{~h} & 0.347663 & -1.030211 & -0.969494 \\ \mathrm{~h} & 1.482219 & -0.364492 & 2.691811 \\ \mathrm{~h} & 4.913002 & 0.501734 & 0.342638 \\ \mathrm{~h} & -4.605777 & 1.127982 & 2.672887 \\ \mathrm{~h} & -3.006058 & 1.864164 & 2.898439 \\ \mathrm{~h} & -3.820910 & 1.977963 & 1.333288 \\ \mathrm{~h} & -3.669179 & -1.094819 & 3.396401 \\ \mathrm{~h} & -2.288105 & -1.757152 & 2.507481\end{array}$




$\begin{array}{llll}\text { h } & -2.062274 & -0.375632 & 3.601720 \\ \text { h } & -6.251123 & 0.838002 & -0.813197 \\ \text { h } & -6.261626 & -0.931902 & -0.913741 \\ \text { h } & -5.718922 & -0.138284 & 0.572696 \\ \text { h } & -4.721083 & 0.930665 & -2.847735 \\ \text { h } & -3.222351 & -0.006498 & -2.806481 \\ \text { h } & -4.769158 & -0.849023 & -2.929167\end{array}$

Transition

$\mathrm{RSTi}\left(\mathrm{N}\left(\mathrm{CH}_{3}\right)_{2}\right)-\mathrm{CH}_{2}---\mathrm{H}--\mathrm{N}\left(\mathrm{CH}_{3}\right)_{2} \underline{\mathrm{RS}} \quad-3815.413597$

$\begin{array}{llll}\text { c } & 0.887607 & 2.998600 & 0.122842 \\ \mathrm{c} & -0.290710 & 2.115626 & 0.568053 \\ \mathrm{~S} & -1.422472 & 1.712856 & -0.857641 \\ \mathrm{Ti} & -2.722664 & -0.080246 & -0.160422 \\ \mathrm{Si} & 2.493495 & 2.216309 & -0.581971 \\ \mathrm{Si} & 4.016357 & 1.333241 & 0.998889 \\ \mathrm{Si} & 3.025176 & -0.481553 & 2.109782 \\ \mathrm{Si} & 2.802346 & -2.224688 & 0.509763 \\ \mathrm{c} & 1.008233 & -2.868843 & 0.306651 \\ \mathrm{c} & 0.022976 & -1.698446 & 0.357859 \\ \mathrm{~S} & -1.647127 & -2.126273 & -0.341451 \\ \mathrm{Si} & 2.351563 & 0.467521 & -2.221924 \\ \mathrm{Si} & 3.731030 & -1.285717 & -1.440266 \\ \mathrm{~h} & -3.366721 & 0.232467 & 1.817383 \\ \mathrm{c} & -3.352420 & -0.818112 & 2.831612 \\ \mathrm{~h} & -3.951972 & -0.234422 & -1.501464 \\ \mathrm{c} & -4.303291 & -0.487155 & -2.877153 \\ \mathrm{c} & -3.521185 & 1.568093 & 2.384609 \\ \mathrm{c} & -4.923224 & 0.007641 & -0.464813 \\ \mathrm{~h} & 4.794356 & -0.438746 & -0.817967 \\ \mathrm{~h} & 3.208909 & 3.323357 & -1.276766 \\ \mathrm{~h} & 2.962209 & 1.045408 & -3.446109 \\ \mathrm{~h} & 3.729433 & -3.260166 & 1.041010 \\ \mathrm{~h} & 3.972678 & -1.032739 & 3.101642 \\ \mathrm{~h} & 0.962344 & 0.044488 & -2.542813 \\ \mathrm{~h} & 4.427793 & -2.181788 & -2.394564 \\ \mathrm{~h} & 4.895784 & 2.199857 & 1.822273 \\ \mathrm{~h} & 1.215194 & 3.592677 & 0.986065 \\ \mathrm{~h} & 0.533477 & 3.727946 & -0.611993 \\ \mathrm{~h} & 0.928658 & -3.385366 & -0.654288 \\ \mathrm{~h} & 0.769389 & -3.606128 & 1.078221 \\ \mathrm{~h} & 0.053947 & 1.200131 & 1.052950 \\ \mathrm{~h} & -0.892204 & 2.657189 & 1.299208 \\ \mathrm{~h} & -0.089309 & -1.354573 & 1.387257 \\ \mathrm{~h} & 0.395919 & -0.862441 & -0.235852 \\ \mathrm{~h} & 1.756660 & -0.139347 & 2.821361\end{array}$




$\begin{array}{llll}\text { h } & 4.917402 & 0.566773 & 0.069536 \\ \text { h } & -4.394291 & 1.621983 & 3.051256 \\ \text { h } & -2.640780 & 1.865446 & 2.968714 \\ \text { h } & -3.657859 & 2.307942 & 1.591823 \\ \text { h } & -4.239461 & -0.758250 & 3.479068 \\ \text { h } & -3.338645 & -1.803730 & 2.364427 \\ \text { h } & -2.467126 & -0.744332 & 3.477503 \\ \text { h } & -5.479152 & 0.946755 & -0.577158 \\ \text { h } & -5.606681 & -0.835537 & -0.299573 \\ \text { h } & -4.330236 & 0.103094 & 0.874235 \\ \text { h } & -4.821752 & 0.377371 & -3.310702 \\ \text { h } & -3.401651 & -0.683556 & -3.459068 \\ \text { h } & -4.969414 & -1.355958 & -2.954163\end{array}$

Adduct2

$\mathrm{RSTi}\left(\mathrm{N}\left(\mathrm{CH}_{3}\right)_{2}\right)_{2}-\mathrm{CH}_{2}-\mathrm{SR}---\mathrm{HN}\left(\mathrm{CH}_{3}\right)_{2}$

$\begin{array}{llll}\text { c } & 0.859989 & 2.984455 & -0.426235 \\ \text { Si } & 2.425134 & 2.066490 & -1.051657 \\ \text { Si } & 2.210194 & 0.027333 & -2.300922 \\ \text { Si } & 3.625862 & -1.545118 & -1.247874 \\ \text { Si } & 2.781822 & -2.075349 & 0.886937 \\ \text { c } & 0.987866 & -2.756552 & 0.902578 \\ \text { c } & -0.036759 & -1.619268 & 0.885560 \\ \text { S } & -1.752474 & -2.207381 & 0.464379 \\ \text { Ti } & -2.779245 & -0.177201 & -0.158379 \\ \text { h } & -3.792237 & -0.663729 & -1.596812 \\ \text { c } & -4.797669 & -0.196235 & -0.727334 \\ \text { c } & -0.298406 & 2.199301 & 0.214264 \\ \text { S } & -1.473382 & 1.556303 & -1.074987 \\ \text { n } & -3.438165 & 0.600157 & 1.876406 \\ \text { c } & -3.998970 & 1.971671 & 1.828516 \\ \text { Si } & 4.019333 & 1.504111 & 0.603327 \\ \text { Si } & 3.079067 & -0.046541 & 2.094552 \\ \text { c } & -2.482935 & 0.446718 & 2.998439 \\ \text { c } & -3.961727 & -1.374828 & -2.838708 \\ \text { h } & 4.715632 & -0.600281 & -0.853932 \\ \text { h } & 3.106851 & 3.006857 & -1.985031 \\ \text { h } & 2.766169 & 0.343327 & -3.642256 \\ \text { h } & 3.737985 & -2.985360 & 1.576317 \\ \text { h } & 4.070600 & -0.393156 & 3.137563 \\ \text { h } & 0.810985 & -0.446225 & -2.467025 \\ \text { h } & 4.281079 & -2.620165 & -2.032666 \\ \text { h } & 4.931868 & 2.511472 & 1.198572 \\ \text { h } & 1.228773 & 3.719902 & 0.300812 \\ \text { h } & 0.470911 & 3.570810 & -1.263889 \\ \text { h } & 0.849440 & -3.394425 & 0.025019\end{array}$




$\begin{array}{llll}\mathrm{h} & 0.831291 & -3.394986 & 1.776828 \\ \mathrm{~h} & 0.073706 & 1.384057 & 0.840141 \\ \mathrm{~h} & -0.862971 & 2.871167 & 0.865298 \\ \mathrm{~h} & -0.049360 & -1.123444 & 1.857336 \\ \mathrm{~h} & 0.241916 & -0.874678 & 0.137658 \\ \mathrm{~h} & 1.847128 & 0.447567 & 2.782636 \\ \mathrm{~h} & 4.876997 & 0.561215 & -0.191535 \\ \mathrm{~h} & -4.429750 & 2.258376 & 2.794515 \\ \mathrm{~h} & -3.208970 & 2.676832 & 1.571940 \\ \mathrm{~h} & -4.764967 & 2.018552 & 1.057799 \\ \mathrm{~h} & -2.925785 & 0.770506 & 3.946897 \\ \mathrm{~h} & -2.183513 & -0.597214 & 3.078687 \\ \mathrm{~h} & -1.597487 & 1.055238 & 2.810132 \\ \mathrm{~h} & -5.326807 & 0.716634 & -1.029916 \\ \mathrm{~h} & -5.484565 & -0.959069 & -0.329885 \\ \mathrm{~h} & -4.215582 & -0.035195 & 2.040282 \\ \mathrm{~h} & -4.449232 & -0.737393 & -3.587006 \\ \mathrm{~h} & -2.991822 & -1.690188 & -3.227841 \\ \mathrm{~h} & -4.588713 & -2.264598 & -2.694106\end{array}$

Product $\mathrm{RSTi}\left(\mathrm{N}_{(}\left(\mathrm{CH}_{3}\right)_{2}\right)-\mathrm{CH}_{2}-\mathrm{SR}$

$-3680.206905$

$\begin{array}{llll}\text { c } & -0.744940 & 2.959850 & -0.808144 \\ \mathrm{Si} & -2.091478 & 2.240300 & 0.357918 \\ \mathrm{Si} & -1.473570 & 0.745538 & 2.134015 \\ \mathrm{Si} & -2.857857 & -1.155734 & 1.917993 \\ \mathrm{Si} & -2.310690 & -2.318956 & -0.054960 \\ \mathrm{c} & -0.475711 & -2.877887 & -0.178781 \\ \mathrm{c} & 0.404464 & -1.774722 & -0.768953 \\ \mathrm{~S} & 2.228050 & -2.122210 & -0.574365 \\ \mathrm{Ti} & 2.999442 & 0.061942 & -0.357393 \\ \mathrm{~h} & 4.550047 & 0.135631 & 0.580750 \\ \mathrm{c} & 4.968227 & 0.385400 & -0.771231 \\ \mathrm{c} & 0.421553 & 2.081055 & -1.287138 \\ \mathrm{~S} & 1.799760 & 2.036569 & -0.034142 \\ \mathrm{Si} & -3.857463 & 1.082199 & -0.705036 \\ \mathrm{Si} & -3.004713 & -0.826820 & -1.772837 \\ \mathrm{c} & 5.391468 & -0.420637 & 1.617755 \\ \mathrm{~h} & -4.087135 & -0.447180 & 1.450547 \\ \mathrm{~h} & -2.710820 & 3.403442 & 1.053721 \\ \mathrm{~h} & -1.833388 & 1.463887 & 3.382902 \\ \mathrm{~h} & -3.258091 & -3.455948 & -0.225911 \\ \mathrm{~h} & -4.104930 & -1.551188 & -2.444560 \\ \mathrm{~h} & -0.024079 & 0.414003 & 2.185293 \\ \mathrm{~h} & -3.261041 & -1.935589 & 3.111988 \\ \mathrm{~h} & -4.952100 & 1.788698 & -1.412184\end{array}$




$\begin{array}{llll}\text { h } & -1.286466 & 3.321826 & -1.691678 \\ \mathrm{~h} & -0.341048 & 3.855306 & -0.326929 \\ \mathrm{~h} & -0.122035 & -3.145699 & 0.820747 \\ \mathrm{~h} & -0.397824 & -3.783067 & -0.787436 \\ \mathrm{~h} & 0.083249 & 1.072784 & -1.538113 \\ \mathrm{~h} & 0.849778 & 2.509741 & -2.194853 \\ \mathrm{~h} & 0.200416 & -1.659235 & -1.833079 \\ \mathrm{~h} & 0.183121 & -0.820683 & -0.282926 \\ \mathrm{~h} & -1.951090 & -0.530592 & -2.789746 \\ \mathrm{~h} & -4.472829 & 0.418079 & 0.493234 \\ \mathrm{~h} & 5.326858 & 1.392569 & -0.994978 \\ \mathrm{~h} & 5.579468 & -0.405833 & -1.222071 \\ \mathrm{~h} & 6.116643 & 0.324552 & 1.962574 \\ \mathrm{~h} & 4.783400 & -0.730349 & 2.469778 \\ \mathrm{~h} & 5.947346 & -1.291141 & 1.246815\end{array}$

